\title{
A Model of Vascular Refilling with Inflammation
}

\author{
Dissertation \\ by \\ Victoria May E. Paguio \\ Supervisor: \\ Em.Univ.-Prof. Dr. Franz Kappel
}

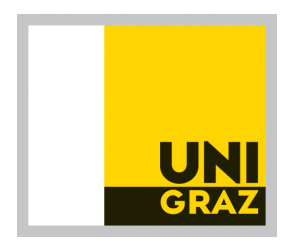

A dissertation presented to the

Faculty of Natural Sciences

in partial fulfillment of the requirements for the degree

Doktor rerum naturalium

Institute for Mathematics and Scientific Computing

Karl-Franzens University of Graz

Graz, October 2016 



\title{
A Model of Vascular Refilling with Inflammation
}

\author{
Victoria May E. Paguio
}

\begin{abstract}
End stage renal disease (ESRD) patients commonly suffer from having excess fluid in the extravascular space. Monitoring the fluid status of patients remains an important aspect in hemodialysis (HD). Recently, de los Reyes et al developed a two-compartment model describing vascular refilling with ultrafiltration (UF) during short-term HD. Ultrafiltration is taking out fluid from the vascular space; while vascular refilling is done through the capillaries and by the lymph, driven by pressure and concentration gradients in the plasma and interstitial spaces.

Cardiovascular disease (CVD) and infection are the most common causes of death among ESRD patients. Inflammation, which is highly prevalent in ESRD patients, is believed to greatly contribute in the progression of CVD. Increased vascular permeability associated with inflammation is likely to influence the capillary wall properties that may cause perturbations affecting capillary refilling during HD.

In this thesis, we present a model which aims to incorporate inflammation to the vascular refilling model of de los Reyes et al with UF during the initial hour of HD. We are interested to know how inflammation may affect the fluid volume and protein concentration dynamics in the plasma and interstitial spaces. We examine how inflammation may change the capillary wall properties, particularly the filtration $\left(L_{p}\right)$ and reflection $(\sigma)$ coefficients. We propose a way to quantify inflammation by considering the concentration of the inflammatory biomarker C-reactive protein (CRP). Functions relating CRP, $L_{p}$, and $\sigma$ are formulated and introduced in the model. Traditional sensitivity functions and subset selection based on asymptotic standard errors were used to aid in parameter identification. Generated measurements for fluid flux and hematocrit were used to obtain estimates for the parameters.
\end{abstract}





\title{
Ein Modell für vaskuläre Nachfüllung bei Vorhandensein von entzündlichen Prozessen
}

\author{
Victoria May E. Paguio
}

\begin{abstract}
Abstrakt
Patienten mit chronischem Nierenversagen im Endstadium (CNES) leiden im allgemeinen unter Flüssigkeisüberschuss im extrazellulärem Bereich. Die Überwachung des Flüssigkeitsstatus von Patienten bleibt ein wichtiger Aspekt der Hämodialyse. Vor kurzem entwickelten de los Reyes u. a. ein Zwei-Kompartment-Model, welches vaskuläre Nachfüllung mittels Ultrafiltration während Kurzzeithämodialyse beschreibt. Ultrafiltration entfernt Flüssigkeit aus dem vaskulären Bereich, während vaskuläre Nachfüllung über die Kapillarien und mittels Lymphe in Reaktion auf Druck- und Konzentrationsgradienten zwischen Plasma und Interstitium erfolgt.

Kardiovaskuläres Versagen und Infektionen sind die häufigsten Todesursachen bei CNES-Patienten. Es wird angenommen, dass entzündliche Prozesse, welche in CNESPatienten weit verbreitet sind, wesentlich zum Fortschreiten von kardiovaskulären Komplikationen beitragen. Vergrösserte vaskuläre Durchlässigkeit bedingt durch entzündliche Prozesse beeinflusst mit hoher Wahrscheinlichkeit die Eigenschaften der Kapillarienwände, wodurch Störungen verursacht werden können, die kapillare Nachfüllung während der Hämodialyse beinflussen.

In der vorliegenden Dissertation präsentieren wir ein Model, welches daraufhin abzielt, entzündliche Prozesse in das Model für kardiovaskuläre Nachfüllung von de los Reyes u. a. mit Ultrafiltration während der ersten Stunde der Hämodialyse einzubauen. Wir sind daran interessiert, zu wissen wie entzündliche Prozesse die Flüssigkeitsvolumina und die Dynamik der Proteinkonzentrationen in Plasma und im Interstitium beeinflussen können. Wir untersuchen, wie entzündliche Prozesse die Eigenschaften der Kapillarienwände, insbesondere den Filtrationskoeffizienten $L_{p}$ und den Reflexionskoeffizienten $\sigma$, beinflussen können. Wir schlagen vor, entzündliche Prozesse durch die Konzentration des inflammatorischen Biomarkers CRP (C-reaktives Protein) zu quantifizieren. Es werden Funktionen entwickelt, welche CRP, $L_{p}$ und $\sigma$ in Verbindung bringen, und in das Model integriert. Traditionelle Sensitivitätsfunktionen und ParameterTeilmengen-Auswahl basierend auf asymptotischen Standardabweichungen wurden verwendet, um die Parameteridentifizierung zu unterstützen. Numerisch erzeugte Messdaten für Flüssigkeitsströme und Hämatokrit wurden verwendet, um Schätzungen für die Parameter zu erhalten.
\end{abstract}





\section{Acknowledgments}

I would like to say my heartfelt thank you to my supervisor Prof. Franz Kappel for his invaluable guidance as I worked on my thesis. Thank you for always giving me encouragement throughout my $\mathrm{PhD}$ studies, and to all the interesting conversations about mathematics, language, history, and life experiences. Your positive outlook in everything has uplifted my spirit during the most challenging times. I would also like to thank the Austrian Agency for International Cooperation in Education and Research (OeAD-GmbH), Center for International Cooperation and Mobility (ICM) for granting me the scholarship TSA Doktorat financed by BMWFW within the framework of the ASEA UNINET; and to OeAD Graz regional office for the assistance during my stay in Graz. I would also like to thank Dr. Peter Kotanko and Dr. Jerry Batzel for sharing their time and expertise to improve this thesis. I am grateful to the Renal Research Institute for its support for this research project. To the faculty and staff of the Institute of Mathematics and Scientific Computing at the University of Graz, thank you for providing me a nice place to work, learn, and meet people; and for being accommodating and always willing to help. To the Institute of Mathematics and the University of the Philippines, thank you for allowing me to pursue my $\mathrm{PhD}$ studies in Austria. Thank you to my family for their love and understanding; and for inspiring me to do my best. To all my friends that I have met here in Graz, thank for your kindness and generosity. My stay here will not be as fun and memorable if I haven't met such wonderful people like you. To my friends back home, thank you for your thoughtfulness; even short conversations made me feel happy and motivated. To Renier, thank you for sharing your time when I need someone to discuss things with, for giving ideas that helped me along the way, for being patient and understanding when I'm being unreasonable, and for the love and encouragement when I feel discouraged. To God, thank for providing me guidance, courage, strength, and resources to accomplish my PhD studies. 



\section{Contents}

List of Figures $\quad$ ix

List of Tables $\quad$ xi

$\begin{array}{llr}1 & \text { Introduction } & 1\end{array}$

1.1 Vascular refilling in hemodialysis $\ldots \ldots \ldots \ldots$

1.1 .1 The microcirculation . . . . . . . . . . . . . . . . . . 3

1.1 .2 Hemodialysis procedure . . . . . . . . . . . . . 6

1.1 .3 A model of vascular refilling . . . . . . . . . . . . . . . . . . . . . . . 8

1.2 Inflammation in ESRD $\ldots \ldots \ldots \ldots$

$1.2 .1 \quad$ Causes of inflammation $\ldots \ldots \ldots \ldots 1$

1.2 .2 Inflammation as a cardiovascular risk factor . . . . . . . . . . 13

1.3 Biomarkers of inflammation . . . . . . . . . . . . . . . . 15

1.3 .1 Inflammatory markers $\ldots \ldots \ldots \ldots \ldots$

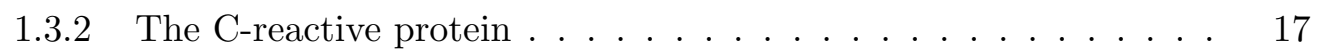

1.4 Effects of inflammation on the capillary wall . . . . . . . . . . . . . . . . 19

1.5 Goal of the study . . . . . . . . . . . . . . . . . 20

2 Mathematical model $\quad 23$

2.1 Data for CRP levels in HD patients . . . . . . . . . . . . . . . . 24

2.2 Data for filtration and reflection coefficients . . . . . . . . . . 25

$\begin{array}{lll}2.3 & \text { Formulating functions for filtration and reflection coefficients }\end{array} \ldots$. . . . 27

$2.4 \quad$ A model of vascular refilling with inflammation . . . . . . . . . . . 30

2.5 Numerical simulations $\ldots \ldots \ldots \ldots$

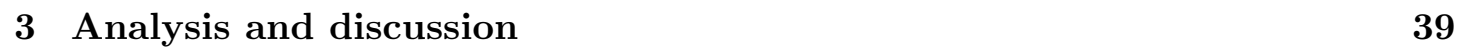

3.1 Sensitivity analysis . . . . . . . . . . . . . . . . . . . . . . . . . . . . . . . . . . . . . . . . . .

3.2 Parameter estimation . . . . . . . . . . . . . . . . . . . 42

3.3 Subset selection algorithm $\ldots \ldots \ldots \ldots$. . . . . . . . . . . . . 45

3.4 Traditional sensitivity functions of the model . . . . . . . . . . . . 46

3.4 .1 Sensitivities with respect to hematocrit . . . . . . . . . . . . . . 47

3.4 .2 Sensitivities with respect to fluid flux . . . . . . . . . . . 54 
3.5 Subset selection results . . . . . . . . . . . . . . . . . . . . . . . . . 58

$3.5 .1 \quad$ Slightly elevated CRP concentration . . . . . . . . . . . . 59

$3.5 .2 \quad$ High CRP concentration . . . . . . . . . . . . . . . . . . 62

$3.6 \quad$ Estimation of best parameter subsets . . . . . . . . . . . . . . . . 66

$3.6 .1 \quad$ Slightly elevated CRP concentration . . . . . . . . . . . . . 68

$3.6 .2 \quad$ High CRP concentration . . . . . . . . . . . . . . . . . 70

3.7 Parameter estimation with two model outputs . . . . . . . . . . . . . 73

3.7.1 Estimates for parameter subsets of length 4 using 2 outputs . . . $\quad 75$

$3.7 .2 \quad$ Estimates for parameter subsets of length 3 using 2 outputs . . . $\quad 77$

3.7 .3 Global search method . . . . . . . . . . . . . . . . . . . . 79

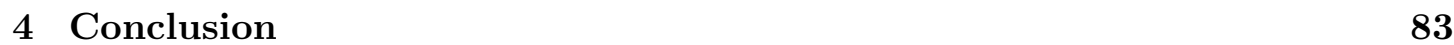

\begin{tabular}{|l|l|}
\hline A Equilibrium Points & 87
\end{tabular}

\begin{tabular}{lr}
\hline Bibliography & 89
\end{tabular} 


\section{List of Figures}

$1.1 \quad$ An illustration of a capillary bed. Adapted from $\mid 15] . \ldots$. . . . . . . . 4

1.2 Types of capillary. Adapted from $[17] \ldots \ldots \ldots \ldots$. . . . . . . . . . . . . . . . 4

1.3 The Starling forces, $P_{i}>0 \ldots \ldots \ldots \ldots \ldots$

$1.4 \quad$ A hemodialysis setup. Adapted from $[20] \ldots \ldots \ldots$. . . . . . . . 7

1.5 Compartment model. Reprinted from $[13] . \ldots \ldots$. . . . . . . . . . 8

$1.6 \quad$ Causes and consequences of inflammation in dialysis patients. Reprinted

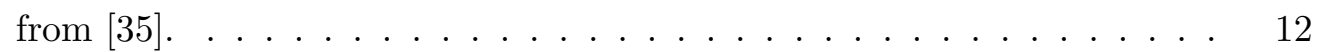

$1.7 \quad$ How inflammation affects PEW in ESRD patients. Adapted from [32]. . 14

1.8 Inflammatory biomarkers associated with cardiovascular risk or mortality

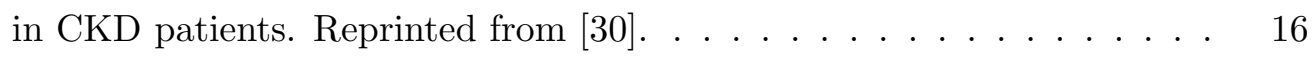

$2.1 \quad$ Graph of the function $(2.2)$ with $a=-9.70, L=22.35$ and $d=66.23$. . $\quad 28$

$2.2 \quad$ Graph of the function (2.4) with $b=0.9058$ and $c=0.0278$. . . . . . . 29

$2.3 \quad$ Plots of the state variable when $[C R P]=0.45 \times 10^{-3} \mathrm{mg} / \mathrm{mL} \ldots . . . \quad 33$

$2.4 \quad$ Plots of the state variables for increasing levels of CRP . . . . . . . . 37

3.2 Sensitivities of hematocrit for different levels of CRP . . . . . . . . . . . 53

3.4 Sensitivities of $J_{v}$ for different levels of CRP . . . . . . . . . . . . . 57 



\section{List of Tables}

1.1 Parameters of the model $(1.2) . \ldots \ldots \ldots \ldots$

2.1 Summary of CRP data, units in $\frac{m g}{L} \ldots \ldots \ldots . \ldots . . \ldots 24$

2.2 Summary of $L_{p}$ data, units in $\frac{m L}{m m H g \cdot m i n} \ldots \ldots \ldots . \ldots . \ldots 26$

$2.3 \quad$ Values used for $L_{p}$ and CRP $\ldots \ldots \ldots \ldots \ldots$. . . . . . . . . . . . . . . . . . . . . . . . . . . .

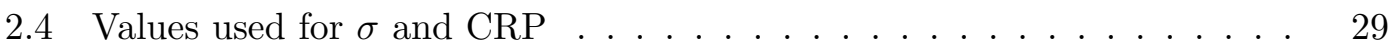

2.5 List of parameters of the model . . . . . . . . . . . . . . . . . . . 31

$2.6 \quad$ Parameter values and initial conditions from [13] . . . . . . . . . . . 32

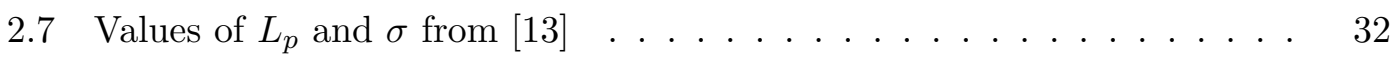

2.8 Nominal values of the parameters in the formulated functions for $L_{p}$ and $\sigma 32$

$2.9 \quad L_{p}$ and $\sigma$ for different values of CRP $\ldots \ldots \ldots \ldots \ldots$

$3.1 \quad$ Initial values for the cases $C R P_{\text {low }}$ and $C R P_{\text {high }} \ldots \ldots \ldots$

$3.2 \quad$ Selection scores of the parameter vector $(a, L, d, b, c)$ for $H c t$ and $J_{v}$ for $C R P_{\text {low }}$ and $C R P_{\text {high }} \ldots \ldots \ldots \ldots \ldots \ldots \ldots \ldots$

$3.3 \quad$ Viable parameter vectors when $p=4,[C R P]=0.00385 \frac{\mathrm{mg}}{\mathrm{mL}}$, and Hct is

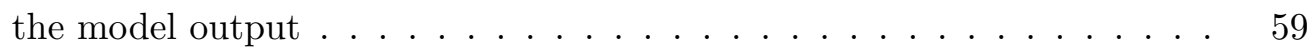

$3.4 \quad$ Viable parameter vectors when $p=3,[C R P]=0.00385 \frac{\mathrm{mg}}{\mathrm{mL}}$, and Hct is the model output . . . . . . . . . . . . . . 60

$3.5 \quad$ Viable parameter vectors when $p=2,[C R P]=0.00385 \frac{\mathrm{mg}}{\mathrm{mL}}$, and Hct is

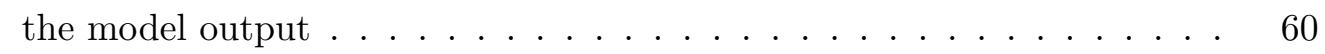

$3.6 \quad$ Viable parameter vectors when $p=4,[C R P]=0.00385 \frac{\mathrm{mg}}{\mathrm{mL}}$, and $J_{v}$ is the model output . . . . . . . . . . . . . . . . . 61

$3.7 \quad$ Viable parameter vectors when $p=3,[C R P]=0.00385 \frac{\mathrm{mg}}{\mathrm{mL}}$, and $J_{v}$ is the model output . . . . . . . . . . . . . . . . . 61

$3.8 \quad$ Viable parameter vectors when $p=2,[C R P]=0.00385 \frac{\mathrm{mg}}{\mathrm{mL}}$, and $J_{v}$ is the model output . . . . . . . . . . . . . . . . . . 62

$3.9 \quad$ Viable parameter vectors when $p=4,[C R P]=0.018 \frac{\mathrm{mg}}{\mathrm{mL}}$, and Hct is the model output . . . . . . . . . . . . . . . . . . 62

3.10 Viable parameter vectors when $p=3,[C R P]=0.018 \frac{\mathrm{mg}}{\mathrm{mL}}$, and Hct is the model output . . . . . . . . . . . . . . . . 63 
3.11 Viable parameter vectors when $p=2,[C R P]=0.018 \frac{m g}{m L}$, and Hct is the

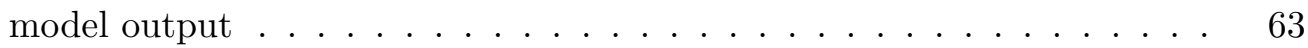

3.12 Viable parameter vectors when $p=4,[C R P]=0.018 \frac{m g}{m L}$, and $J_{v}$ is the

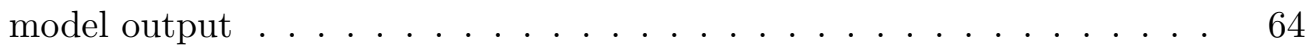

3.13 Viable parameter vectors when $p=3,[C R P]=0.018 \frac{m g}{m L}$, and $J_{v}$ is the

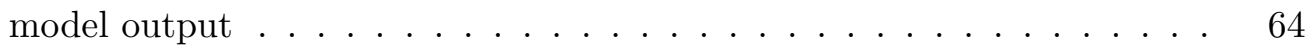

3.14 Viable parameter vectors when $p=2,[C R P]=0.018 \frac{m g}{m L}$, and $J_{v}$ is the

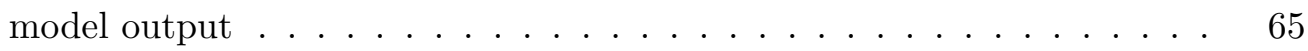

3.15 Best parameter subsets of lengths 2,3 and 4 based on the selection score $\alpha(\theta)$ for the model outputs $H c t$ and $J_{v} \ldots \ldots \ldots \ldots \ldots \ldots$

3.16 Estimates for $(a, L, d, b, c)$ when $[C R P]=0.00385 \mathrm{mg} / \mathrm{mL}$ and $H c t$ is the

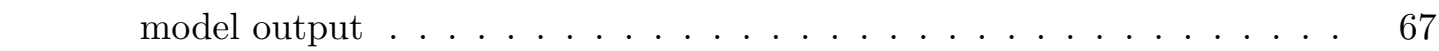

3.17 Estimates for $(a, L, d, b, c)$ when $[C R P]=0.018 \mathrm{mg} / \mathrm{mL}$ and $H c t$ is the

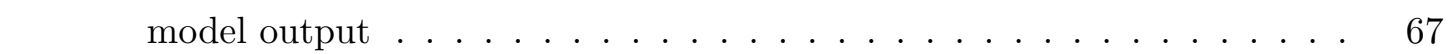

3.18 Estimates for $(a, L, d, b, c)$ when $[C R P]=0.018 \mathrm{mg} / \mathrm{mL}$ and $J_{v}$ is the

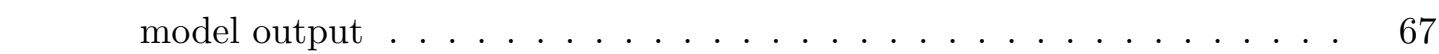

3.19 Estimates for $(a, L, b, c)$ when $[C R P]=0.00385 \mathrm{mg} / L$ and $H c t$ as the

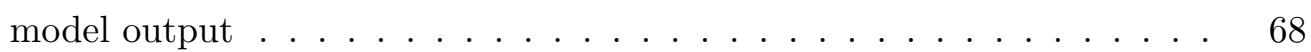

3.20 Estimates for $(L, b, c)$ when $[C R P]=0.00385 \mathrm{mg} / \mathrm{L}$ and $H c t$ as the model

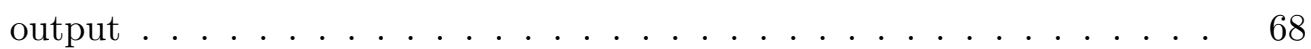

3.21 Estimates for $(L, b)$ when $[C R P]=0.00385 \mathrm{mg} / L$ and $H c t$ as the model

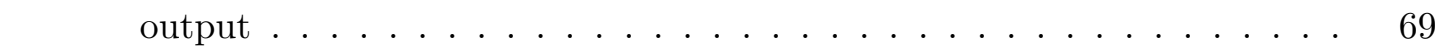

3.22 Estimates for $(a, L, b, c)$ when $[C R P]=0.00385 \mathrm{mg} / L$ and $J_{v}$ as the model output . . . . . . . . . . . . . . . . . . 69

3.23 Estimates for $(a, L, c)$ when $[C R P]=0.00385 \mathrm{mg} / L$ and $J_{v}$ as the model

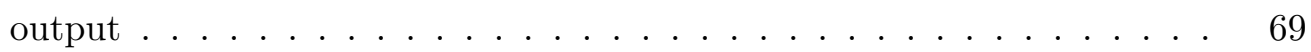

3.24 Estimates for $(L, b)$ when $[C R P]=0.00385 \mathrm{mg} / L$ and $J_{v}$ as the model

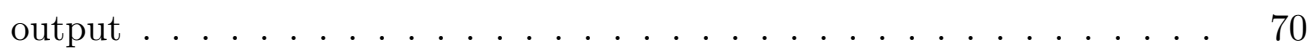

3.25 Estimates for $(a, L, b, c)$ when $[C R P]=0.018 \mathrm{mg} / L$ and $H c t$ as the model

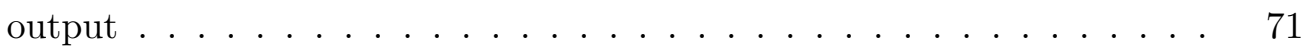

3.26 Estimates for $(L, b, c)$ when $[C R P]=0.018 \mathrm{mg} / \mathrm{L}$ and $H c t$ as the model

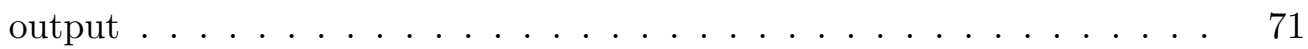

3.27 Estimates for $(L, b)$ when $[C R P]=0.018 \mathrm{mg} / \mathrm{L}$ and $H c t$ as the model

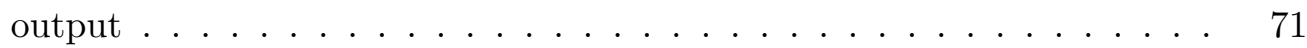

3.28 Estimates for $(a, d, b, c)$ when $[C R P]=0.018 \mathrm{mg} / L$ and $J_{v}$ as the model

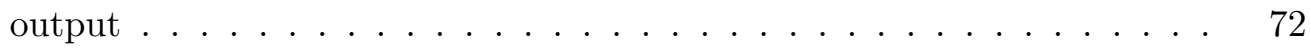

3.29 Estimates for $(L, b, c)$ when $[C R P]=0.018 \mathrm{mg} / L$ and $J_{v}$ as the model

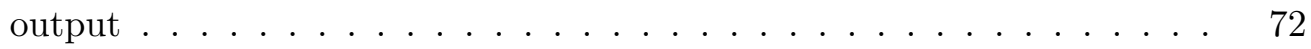

3.30 Estimates for $(a, b, c)$ when $[C R P]=0.018 \mathrm{mg} / L$ and $J_{v}$ as the model

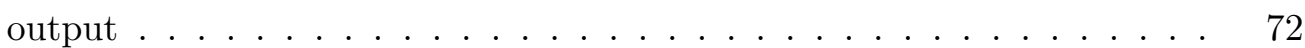


3.31 Estimates for $(d, b, c)$ when $[C R P]=0.018 \mathrm{mg} / L$ and $J_{v}$ as the model

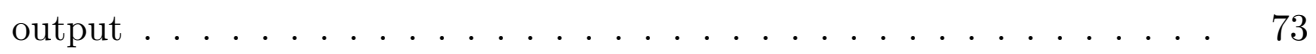

3.32 Estimates for $(L, b)$ when $[C R P]=0.018 \mathrm{mg} / L$ and $J_{v}$ as the model output 73

3.33 Estimates for $(a, L, d, b, c)$ when $[C R P]=0.00385 \mathrm{mg} / \mathrm{mL}$ using two

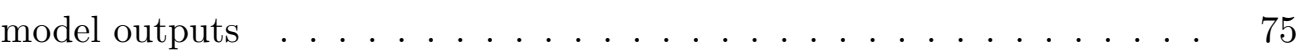

3.34 Estimates for $(a, L, d, b, c)$ when $[C R P]=0.018 \mathrm{mg} / \mathrm{mL}$ using two model

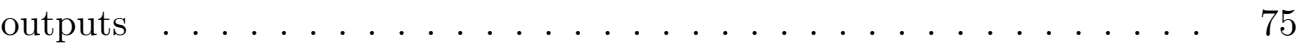

3.35 Estimates for $(a, L, b, c)$ when $[C R P]=0.00385 \mathrm{mg} / \mathrm{mL}$ using two model

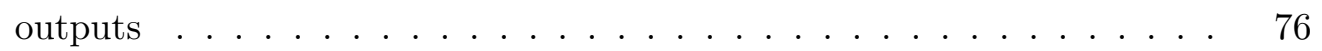

3.36 Estimates for $(a, L, b, c)$ when $[C R P]=0.018 \mathrm{mg} / \mathrm{mL}$ using two model

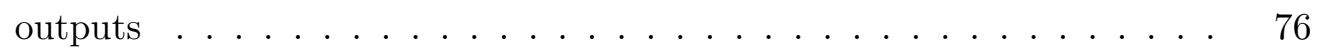

3.37 Estimates for $(a, d, b, c)$ when $[C R P]=0.018 \mathrm{mg} / \mathrm{mL}$ using two model

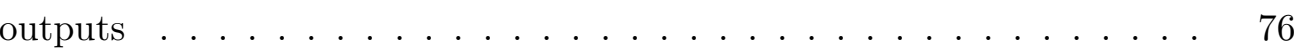

3.38 Estimates for $(a, L, d, b)$ when $[C R P]=0.00385 \mathrm{mg} / \mathrm{mL}$ using two model

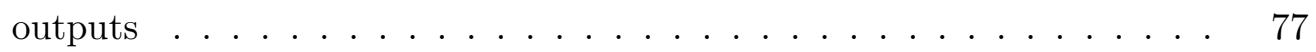

3.39 Estimates for $(a, L, d, b)$ when $[C R P]=0.018 \mathrm{mg} / \mathrm{mL}$ using two model

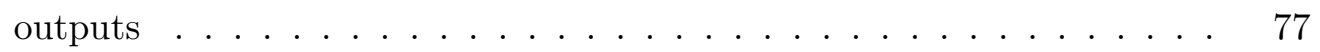

3.40 Estimates for $(a, L, d, c)$ when $[C R P]=0.00385 \mathrm{mg} / \mathrm{mL}$ using two model

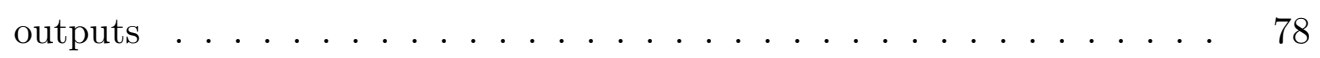

3.41 Estimates for $(a, L, d, c)$ when $[C R P]=0.018 \mathrm{mg} / \mathrm{mL}$ using two model

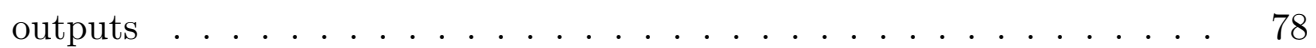

3.42 Estimates for $(L, b, c)$ when $[C R P]=0.00385 \mathrm{mg} / \mathrm{mL}$ using two model

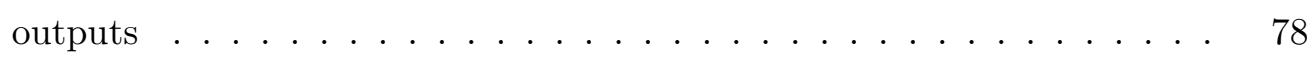

3.43 Estimates for $(L, b, c)$ when $[C R P]=0.018 \mathrm{mg} / \mathrm{mL}$ using two model

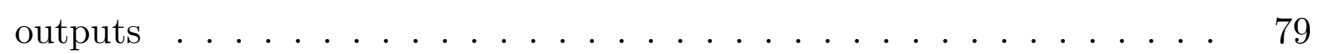

3.44 Estimates for $(a, L, c)$ when $[C R P]=0.00385 \mathrm{mg} / \mathrm{mL}$ using two model

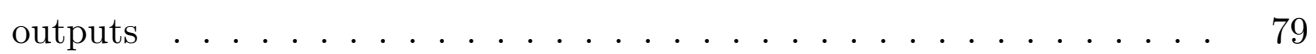

3.45 Estimates for $(a, L, c)$ when $[C R P]=0.018 \mathrm{mg} / \mathrm{mL}$ using two model

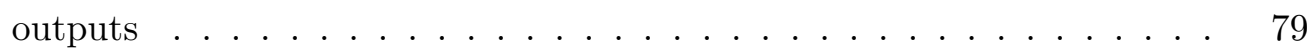

3.46 Estimates for different parameter subsets using GA and fminsearch; $[C R P]=$ $0.00385 \mathrm{mg} / \mathrm{mL}$ using two model outputs; bounds are $15 \%$ from the nominal values . . . . . . . . . . . . . . . . . . 80

3.47 Estimates for different parameter subsets using GA and fminsearch; $[C R P]=$ $0.00385 \mathrm{mg} / \mathrm{mL}$ using two model outputs; bounds are $30 \%$ from the nominal values . . . . . . . . . . . . . . . . . . . 81

3.48 Estimates for different parameter subsets using GA and fminsearch; $[C R P]=$ $0.018 \mathrm{mg} / \mathrm{mL}$ using two model outputs; bounds are $15 \%$ from the nominal values 
3.49 Estimates for different parameter subsets using GA and fminsearch; $[C R P]=$ $0.018 \mathrm{mg} / \mathrm{mL}$ using two model outputs; bounds are $30 \%$ from the nominal values 


\section{Chapter 1}

\section{Introduction}

Removal of metabolic waste products and toxins, as well as regulation of fluid volume and composition, are among the important functions of the kidneys [1]. Dysfunctional kidneys not only cause retention of substances such as urea, uric acid and creatinine, but also cause retention of fluid. Uremia, the state of having elevated levels of such substances, normally accompanies kidney disease. Moreover, patients with kidney disease are often experiencing fluid overload. Excess fluid may accumulate in the extravascular space, which includes both interstitial and intracellular spaces, or in the intravascular space.

Kidney disease may be acute or chronic, depending on whether its development over time is rapid or gradual. In acute kidney disease, kidney function decreases or stops abruptly, but may eventually return to its normal state. Some causes of this sudden dysfunction are hemorrhage, abnormalities within the kidney itself, or kidney stones. On the other hand, chronic kidney disease progresses gradually over time and causes irreversible damage. Kidney Disease Improving Global Outcomes (KDIGO) defines chronic kidney disease (CKD) as "abnormalities in kidney structure or function that is present for at least three months and has implications for health" [2]. There are various blood and urine tests to measure kidney function, which are based on the levels or clearance rates of substances like urea nitrogen, creatinine, and albumin. One such test involves the glomerular filtration rate (GFR). The GFR of normal healthy kidneys is greater than or equal to $90 \mathrm{~mL} / \mathrm{min} / 1.73 \mathrm{~m}^{2}$. According to KDIGO, a GFR less than $60 \mathrm{~mL} / \mathrm{min} / 1.73 \mathrm{~m}^{2}$ with or without kidney damage, also defines chronic kidney disease. Other indications of kidney damage include albuminuria, urine and electrolyte abnormalities, abnormalities seen from imaging, and history of kidney transplantation 2. Chronic kidney disease is progressive and a precursor to the worst stage of CKD, which is called end-stage renal disease (ESRD). Individuals with ESRD have almost or complete kidney failure and would need renal replacement therapy such as hemodialysis (HD), peritoneal dialysis (PD), or kidney transplantation in order to live.

Chronic kidney disease and ESRD are commonly caused by hypertension and di- 
abetes [1, 3]. Mortality rate of ESRD patients undergoing renal replacement therapy reaches about $20 \%$ per year and most of these patients die within 5 years [4]. Cardiovascular disease (CVD) and infection are the most common causes of death of ESRD patients. Mortality rates due to CVD and infection are up to $50 \%$ and $20 \%$, respectively, of the total deaths [5]. Cardiovascular disease occurs two times more in ESRD patients than in the general population and it progresses twice the rate in CKD patients than the general population [6. Chronic kidney disease is considered as an independent risk factor for CVD. Likewise, CVD seems to be a risk factor for CKD [7, 8, 9]. This means that deteriorating kidney state and worsening CVD are interrelated, wherein one might be contributing to the other's decline and vice versa.

Because of the high annual mortality and short survival rate, improvements on the hemodialysis process and dosage still continue. It has already been recognized that although traditional risk factors such as age, male sex, diabetes mellitus, hypertension, dyslipidemia, left ventricular hypertrophy and smoking are very common in ESRD patients, these do not entirely account for the high cardiovascular risk in ESRD patients [10, 8]. Nontraditional or novel risk factors such as inflammation, oxidative stress, endothelial dysfunction, vascular calcification, malnutrition, and protein-energy wasting also appear to contribute greatly to the CVD development of ESRD patients [8]. For instance, chronic inflammation, endothelial dysfunction, and increased oxidative stress may cause accelerated atherosclerosis promoting an increased cardiovascular risk in ESRD patients [11]. To distinguish which among the traditional and nontraditional factors cause CVD is almost impossible because normally, these risk factors coexist and are interrelated [12].

Recently, de los Reyes et al. [13] published a model of vascular refilling during ultrafiltration (UF) in hemodialysis. In their paper, they looked at the dynamics of the plasma and interstitial volumes, as well as the dynamics of the protein concentrations in the plasma and interstitial spaces, when both vascular refilling and UF are taking place. Meanwhile, there is accumulating evidence that chronic inflammation aggravates CVD in ESRD patients undergoing hemodialysis. This study adopts the model by de los Reyes et al. 13 to include the effects of inflammation by examining more closely the parameters for filtration rate and osmotic reflection coefficient. These two parameters are directly related to the capillary wall properties, which would naturally be affected in the presence of inflammation. The study intends to investigate how inflammation may possibly affect the vascular refilling dynamics during UF in hemodialysis. To incorporate inflammation, a particular inflammatory biomarker called C-reactive protein (CRP) is considered. Its level in the blood increases in response to inflammation and is known to be a good predictor of future cardiovascular events [14.

The following sections in this chapter provides some information on vascular refilling and hemodialysis processes; the prevalence of inflammation in ESRD, as well as its causes 
and consequences; the markers of inflammation particularly, the C-reactive protein; and the known effects of inflammation on the capillary wall. In chapter 2, we discuss the development of the model. In chapter 3 , we analyze the model using traditional sensitivity analysis and parameter estimation. Subset selection is also done to improve estimates. Chapter 4 summarizes the study and gives the conclusion.

\subsection{Vascular refilling in hemodialysis}

\subsubsection{The microcirculation}

The site where exchange of materials between the plasma and the tissue space occurs across a capillary wall. A capillary is a small blood vessel composed of a single layer of endothelial cells surrounded outside by a basal lamina. Their walls consist of small pores and other pathways and are highly permeable to small solutes, gases, and water. Generally, the pores of the capillaries are slightly smaller than plasma proteins. Hence, proteins are not able to pass through. Note however, that there are different types of capillaries and their permeabilities to various molecules differ.

Capillaries are the smallest blood vessels with a wall thickness of around $0.5 \mu \mathrm{m}$ and an internal diameter of about 4-9 $\mu \mathrm{m}$, which is a little smaller than a red blood cell [1. They are connected to arterioles via precapillary sphincters on one end and to venules on the other end. Precapillary sphincters are smooth muscle fibers that can open and close, regulating the flow of blood from the arteriole to the capillary. Moreover, capillaries form a network called a capillary bed or capillary network, wherein nutrients and gases are delivered to the tissues from the blood; and waste products are taken up from the tissues and delivered back to the blood stream. One can see in figure 1.1 a capillary bed connected to an arteriole on the left and a venule on the right; tissue cells and interstitial fluid are in the background surrounding the vessels; and lymphatic capillaries are intertwined with the blood capillaries are in the center. Blood flows from the arterioles to the capillaries, regulated by the precapillary sphincters. The precapillary sphincters deliver blood intermittently to the capillaries and consequently, to the tissue cells [1]. When the sphincters are closed, blood does not flow to the capillary, while if they are open, blood is allowed to flow through the capillary. Because of the action of the precapillary sphincters, capillaries are not always filled with blood. But more sphincters open to supply the capillaries with blood when there is increased demand by the tissues, just as in the case of inflammation. After the exchange of materials on the capillary bed across the capillary walls, blood then continues to flow from the capillaries to the postcapillary venules. Meanwhile, lymphatic capillaries collect proteins that leaked out and excess fluid from the interstitial space, and carry them back to the vascular space. According to Guyton and Hall [1, the peripheral circulation of the whole body has about 10 billion capillaries, which forms a surface area of about 
500-700 $\mathrm{m}^{2}$. Hence, these numerous and well-connected capillaries provide an effective and efficient way of transporting substances to and from the cells.

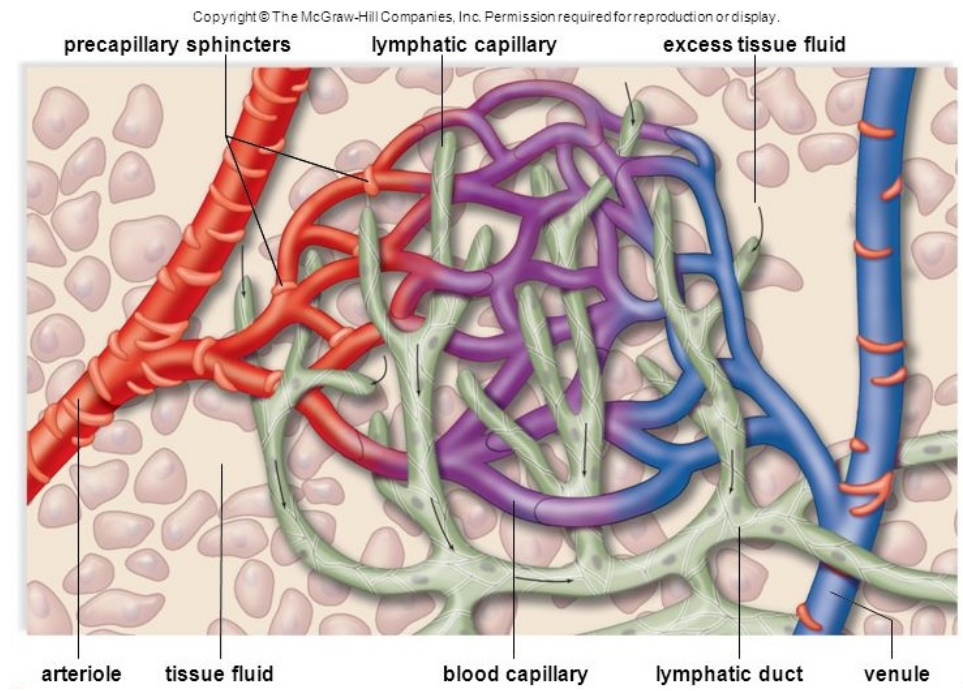

Figure 1.1: An illustration of a capillary bed. Adapted from 15.

There are three types of capillaries: continuous, fenestrated, and sinusoidal or discontinuous. Continuous capillaries are the most common and most abundant. They allow small molecules, ions and water to pass through the gaps between endothelial cells, which are called intercellular clefts. Fenestrated capillaries appear to have fenestrations or windows on the capillary membrane. They are more permeable than continuous capillaries and are found in endocrine and exocrine glands, gut, and kidney [16]. Sinusoidal or discontinuous capillaries have the biggest pores, allowing bigger molecules such as proteins to pass through the capillary membrane. This type of capillary is found in the liver, spleen, and bone marrow [16]. Figure 1.2 shows the different types of capillaries. Regardless of the type of capillary, diffusion and convection facilitate the exchange of fluid and molecules across the membrane.
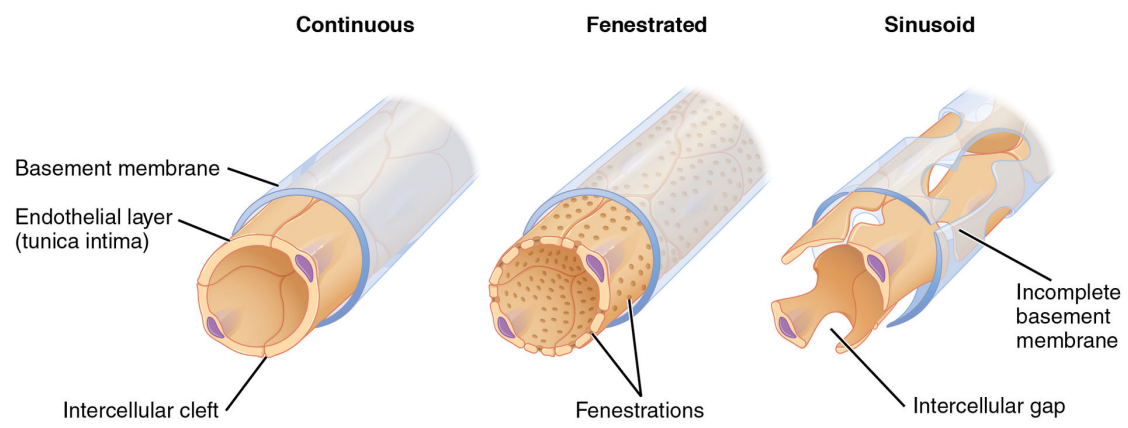

Figure 1.2: Types of capillary. Adapted from 17.

The fluid flow across the capillary wall is governed by hydrostatic and osmotic pres- 
sure gradients, which cause filtration and reabsorption of fluid across the membrane. There are four types of such pressures: capillary hydrostatic pressure, interstitial hydrostatic pressure, plasma oncotic pressure, and interstitial oncotic pressure. These four pressures are referred to as the Starling forces. Capillary hydrostatic pressure $\left(P_{c}\right)$ is the pressure inside the capillaries that forces the fluid to move out of the capillary into the extravascular space. Conversely, interstitial hydrostatic pressure $\left(P_{i}\right)$ is the pressure of fluid in the tissue space. If $P_{i}$ is positive, then it forces the fluid from the interstitial space to move into the capillaries. When $P_{i}$ is negative, the net effect is filtration rather than reabsorption of fluid. The average functional capillary pressure is around 17 $m m H g$, while the average interstitial hydrostatic pressure in loose subcutaneous tissue is about $-3 \mathrm{mmHg}$ [1]. The oncotic or colloid osmotic pressure is the force exerted by the proteins. Plasma oncotic pressure $\left(\pi_{p}\right)$ is the force due to proteins in the plasma, which causes fluid to move into the capillary. On the other hand, interstitial oncotic pressure $\left(\pi_{i}\right)$ represents the force exerted by the proteins in the interstitial space that causes fluid to be filtered out of the capillary. Interstitial fluid is normally protein-poor compared to the plasma. The average plasma oncotic pressure is believed to be about $28 \mathrm{~mm} \mathrm{Hg}$ and the average interstitial oncotic pressure is about $8 \mathrm{mmHg}$ [1]. Figure 1.3 illustrates the Starling forces, where $P_{c}$ and $\pi_{i}$ promote filtration of fluid out of the capillary, while $P_{i}>0$ and $\pi_{p}$ promote reabsorption of fluid into the capillary.

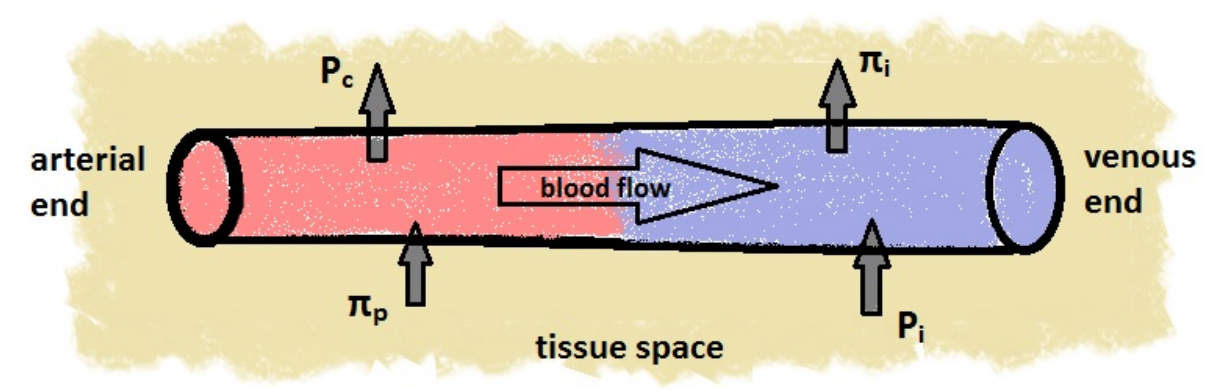

Figure 1.3: The Starling forces, $P_{i}>0$

The net flow of fluid across the capillary wall at time $t$, denoted by $J_{v}(t)$, is given by

$$
J_{v}(t)=L_{p}\left(\sigma\left(\pi_{p}(t)-\pi_{i}(t)\right)-\left(P_{c}(t)-P_{i}(t)\right)\right),
$$

where $L_{p}$ is the filtration coefficient equal to the product of hydraulic conductivity and surface area and $\sigma$ is the osmotic reflection coefficient. The filtration coefficient $L_{p}$ varies directly with the number and size of pores and inversely with the viscosity of the fluid and thickness of the membrane 18. Naturally, the filtration coefficient of the walls of fenestrated capillaries is more than those of continuous capillaries but less than those of discontinuous capillaries. The osmotic reflection coefficient $\sigma$ describes the permeability of a protein to a capillary membrane. It has a value between 0 and 1 , where 0 means no 
reflection and the protein can freely pass through the membrane, while 1 means perfect reflection or total impermeability of the protein to the membrane. The parameters $L_{p}$ and $\sigma$ represent the properties of the capillary wall. How these parameters are changed by the presence of inflammation is a main subject of this work and is examined more closely in the next chapter.

The traditional view concerning the Starling forces is that filtration is greater at the arterial end, while reabsorption is favored at the venular end of the capillary. Also, as blood flows along the capillary, there is a small net filtration of fluid that is returned to the circulation via the lymph. However, this view has been recently reconsidered saying that there is primarily filtration throughout the entire length of the capillary. This means that there is greater filtration compared to the traditional view. This also means that the lymphatic system plays a bigger role than previously thought, in returning filtered fluid and molecules back to the circulation [19].

\subsubsection{Hemodialysis procedure}

Hemodialysis is one of the renal replacement therapies available for individuals with kidney disease. Besides the inability of patients with CKD and ESRD to naturally remove metabolic waste products, another major problem that they experience is the retention of fluid that should have been taken out from the system by the kidneys. Having fluid overload is prevalent among HD patients. The excess fluid stays in the vascular space or goes to the tissue and intracellular spaces. The main goal of hemodialysis is the removal of the excess fluid and waste products. Hemodialysis is usually done three times a week and each session lasts for about four hours.

Figure 1.4 shows a typical hemodialysis setup. A patient is connected to a dialysis machine via a vascular access, which in this case is on the arm. Blood is taken out from an artery of a patient, passed though a dialyzer where wastes and fluid are removed, then the "clean" blood is returned back to the patient through a vein. A dialyzer, seen on the right of figure 1.4 is a filter containing hollow tubes with semipermeable membranes. The blood flows through these tubes and a fluid called dialysate flows outside the tubes in a countercurrent manner. The dialysate is prepared such that its composition is ideal to obtain normal blood components after diffusion. Because of pressure and solute concentration gradients, exchange of materials between the blood and the dialysate and removal of water from the plasma are possible. Diffusion facilitates the solute exchange, while ultrafiltration enables the removal of water. Around four liters of fluid is typically removed from a patient in one dialysis session. After passing through the dialyzer, the blood containing less water and less toxins goes back to the patient and the used dialysate is discarded.

As an effect of the removal of water in the plasma, excess fluid that is in the tissue 


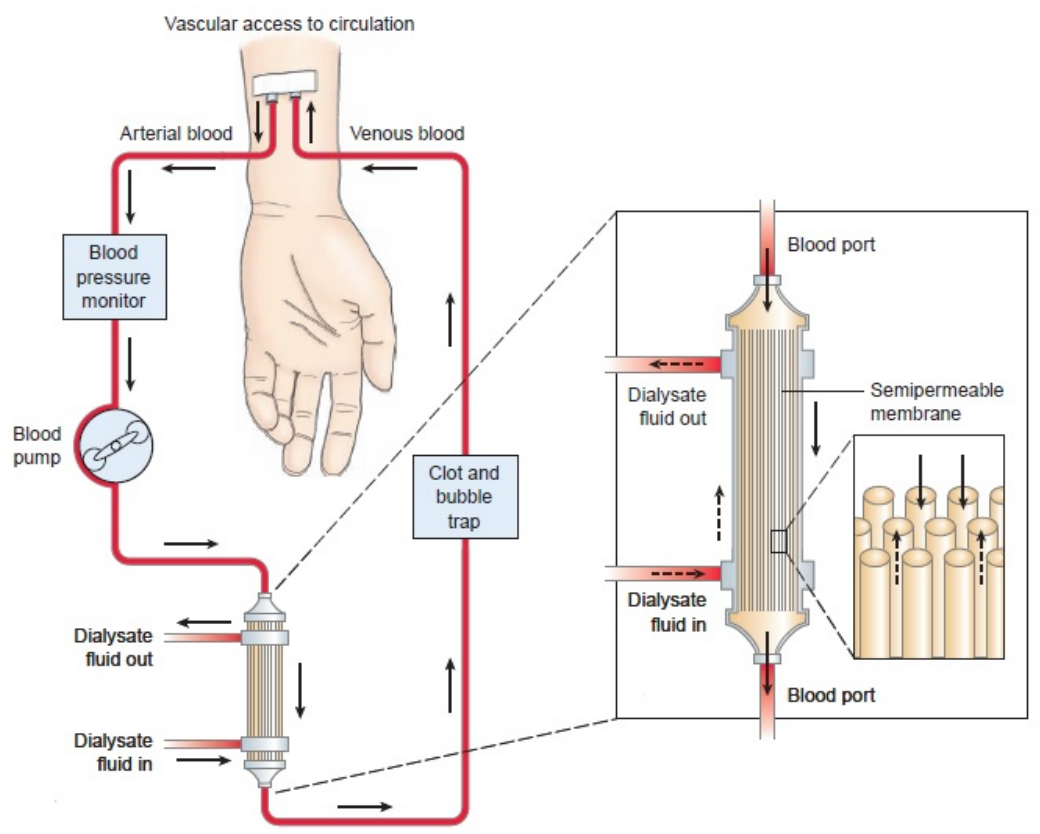

Figure 1.4: A hemodialysis setup. Adapted from 20

and intracellular spaces will tend to move into the vascular space in a process called vascular refilling. The vascular space can be refilled through the lymph flow or through the flow across the capillary walls. In this study, the lymph flow is assumed constant and vascular refilling will mainly refer to the flow across the capillary walls.

Vascular refilling is governed by the differences in hydrostatic and oncotic pressures 21. It is also dependent on a number of hemodialysis- and patient-related factors such as ultrafiltration rate, amount of fluid overload, properties of the capillary wall, and body size [13]. High ultrafiltration rate may cause the circulating blood volume to drop quickly and consequently, may lead to hypovolemia and hypotension. On the other hand, inadequate removal of fluid means that a patient will remain in a state of overhydration even after HD and this causes persistent high blood pressure 22]. Over time, these stresses contribute to various cardiovascular problems developed by ESRD patients. The presence of inflammation may directly affect some properties of the capillary wall such as its permeability. Therefore, inflammation may also affect the rate of vascular refilling in the capillaries.

It is important to maintain blood volume within a normal range as it also affects blood pressure. Regulation of blood volume is a problem for ESRD patients because their kidneys are compromised. For HD patients, changes in blood volume depend on a number of factors particularly, ultrafiltration rate and vascular refilling rate. Absolute blood volume can be measured using dilution of tracers such as dyes or radioisotopes. In this method, a fixed concentration of tracer is injected and allowed to mix with the blood. After mixing, its concentration is again measured and this provides a way 
to determine the absolute blood volume [23]. However, this method is impractical for patients undergoing dialysis regularly. Other blood volume monitors (BVM) are based on the idea that some blood components, such as proteins and hemoglobin, remain in the vascular space. These provide relative blood volume measurements based on the ratios of the concentrations of a blood component [22]. The need for monitoring blood volume continuously and non-invasively is important in regulating fluid status during hemodialysis treatment and preventing cardiovascular disease progression.

\subsubsection{A model of vascular refilling}

Very recently, de los Reyes et al. published a vascular refilling model during ultrafiltration in hemodialysis. In their paper, a two-compartment model, shown in figure 1.5 , is developed. It reflects short-term changes in relative blood volume (RBV) due to UF and vascular refilling from the capillaries and the lymph. Hematocrit data from a Crit-Line Monitor (CLM) during the initial hour of HD are used to identify some parameters.

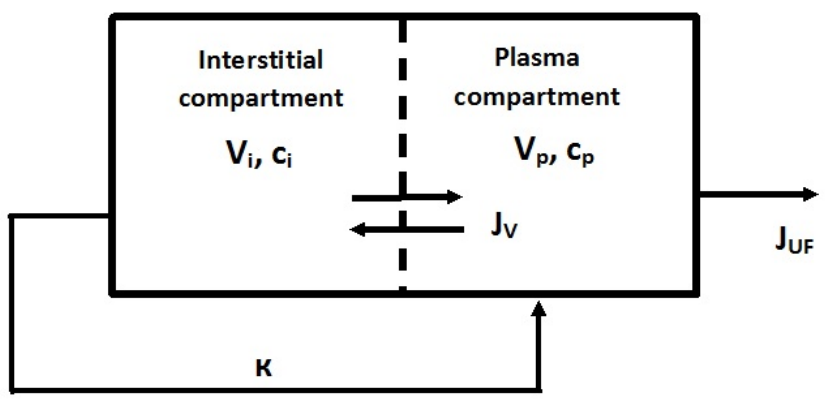

Figure 1.5: Compartment model. Reprinted from 13 .

In the model, it is assumed that fluid and proteins are exchanged between the interstitial and plasma compartments across a semipermeable membrane. Also, only fluid is removed from the plasma compartment by ultrafiltration in the dialyzer; that is, no proteins are taken out from this compartment. The lymph flow, with rate $k$, goes from the interstitial compartment to the plasma compartment. For the initial hour of treatment, lymph flow is assumed constant; but for longer periods of treatment, it may change considerably. Refer to 13 for a detailed discussion of the development of the model and for a complete list of assumptions.

We now present in 1.2 the model equations as written in the paper by de los Reyes et al. The state variables considered are plasma volume $\left(V_{p}\right)$, interstitial volume $\left(V_{i}\right)$, protein concentration in the plasma compartment $\left(c_{p}\right)$, and protein concentration in the interstitial compartment $\left(c_{i}\right)$. The model describes the dynamics of vascular refilling 
with ultrafiltration for a period of one hour after the start of the HD treatment. In this case, it can be assumed that lymph flow and hydrostatic pressures are constant.

$$
\begin{aligned}
\frac{d V_{p}}{d t} & =J_{v}+\kappa-J_{U F} \\
\frac{d c_{p}}{d t} & =\frac{J_{s}-c_{p}\left(J_{v}+\kappa-J_{U F}\right)}{V_{p}} \\
\frac{d V_{i}}{d t} & =-J_{v}-\kappa \\
\frac{d c_{i}}{d t} & =\frac{-J_{s}+c_{i}\left(J_{v}+\kappa\right)}{V_{i}}
\end{aligned}
$$

where

$$
\begin{aligned}
J_{v}(t) & =L_{p}\left(\sigma\left(\pi_{p}(t)-\pi_{i}(t)\right)-\left(P_{c}-P_{i}\right)\right) \\
\pi_{p}(t) & =a_{p, 1} c_{p}(t)+a_{p, 2} c_{p}(t)^{2} \\
\pi_{i}(t) & =a_{i, 1} c_{i}(t)+a_{i, 2} c_{i}(t)^{2} \\
J_{s}(t) & = \begin{cases}J_{v}(t)(1-\sigma)\left(c_{i}(t)-\frac{c_{p}(t)-c_{i}(t)}{e^{x(t)}-1}\right)+\alpha \kappa, & J_{v}(t)>0 \\
-P_{S}\left(c_{p}(t)-c_{i}(t)\right)+\alpha \kappa, & J_{v}(t)=0 \\
J_{v}(t)(1-\sigma)\left(c_{p}(t)-\frac{c_{p}(t)-c_{i}(t)}{e^{x(t)}-1}\right)+\alpha \kappa, & J_{v}(t)<0\end{cases} \\
x(t) & =\frac{J_{v}(t)(1-\sigma)}{P_{S}}
\end{aligned}
$$

The change in volume of the plasma compartment over time is dependent on the ultrafiltration rate $J_{U F}$, fluid flux $J_{v}$, and lymph flow $\kappa$, while the change in volume of the interstitial compartment over time depends on $J_{v}$ and $\kappa$. The fluid flux $J_{v}(t)$ describes the rate of fluid flow across the membrane at time $t$ and is influenced by the Starling forces. The changes in protein concentrations of both compartments over time are dependent on the protein flux $J_{s}$ and the changes in compartment volumes. The protein flux $J_{s}(t)$ represents the movement of proteins between the compartments at time $t$ and is influenced by diffusion and convection of proteins across the capillary membrane, and protein concentration in the lymph. Note that flow toward the plasma compartment is set as positive. Table 1.1 lists the parameters appearing in 1.2 .

The model equation written above is the basis for this study. Inflammation per se cannot be measured directly during HD [13]. We would like to incorporate inflammation to this model by considering inflammatory biomarkers, which are measurable. The parameters representing the capillary wall properties, namely $L_{p}$ and $\sigma$, are reconsidered and examined with respect to a biomarker. In this way, we can quantify the level of inflammation and investigate how it may affect the vascular refilling dynamics during UF. 


\begin{tabular}{|l|l|}
\hline Parameter & Meaning \\
\hline$L_{p}$ & filtration coefficient \\
$\sigma$ & reflection coefficient \\
$P_{c}$ & hydrostatic capillary pressure \\
$P_{i}$ & hydrostatic interstitial pressure \\
$\pi_{p}$ & plasma colloid osmotic pressure \\
$\pi_{i}$ & interstitial colloid osmotic pressure \\
$\kappa$ & lymph flow rate \\
$J_{U F}$ & ultrafiltration rate \\
$a_{p, 1}, a_{p, 2}$ & coefficients appearing in the equation for $\pi_{p}$ \\
$a_{i, 1}, a_{i, 2}$ & coefficients appearing in the equation for $\pi_{i}$ \\
$x(t)$ & Peclet number \\
$P S$ & permeability-surface area product \\
$\alpha$ & protein concentration in the lymph \\
\hline
\end{tabular}

Table 1.1: Parameters of the model 1.2.

\subsection{Inflammation in ESRD}

Inflammation is the body's response to tissue injury, which may be caused by bacteria, virus, trauma or various irritants. It involves changes and release of multiple substances to target the stimulus and protect the undamaged tissues [1, 24]. Chronic inflammation is a prolonged state of inflammatory response caused by a persistent stimulus that becomes harmful and destroys tissues 24,25 .

Systemic low-grade inflammation is now an established feature common to CKD and ESRD patients. According to Stenvinkel [26, 27], between $30 \%-70 \%$ of ESRD patients on hemodialysis have inflammation. This is based on the fact that ESRD patients show activated acute phase response as evidenced by increased serum levels of acute phase reactants such as C-reactive protein (CRP), fibrinogen, and lipoprotein(a) $(\operatorname{Lp}(\mathrm{a}))$ [28]; and proinflammatory cytokines such as interleukin-1 (IL-1), interleukin-6 (IL-6), and tumor necrosis factor $\alpha(\mathrm{TNF}-\alpha)[29,30,31$. Decreased levels of albumin 32 or anti-inflammatory cytokines like IL-10 are also indicators of activated inflammatory response. Some of these biomarkers not only serve as signals to detect the presence of inflammation, but may also even be risk factors for CVD [33]. In this study, elevated level of proinflammatory markers is synonymous to having systemic inflammation.

It is recognized that chronic inflammation is strongly linked to protein energy wasting (PEW), accelerated atherosclerosis, immune, metabolic and nutritional dysfunctions, and vascular calcification 24, 30, 31]. Inflammation is believed to aggravate these processes leading to an increased risk of cardiovascular disease and infection 31. Moreover, there are studies which observed that persistent low-grade inflammation is common in 
ESRD and CKD patients even before undergoing renal replacement therapy [6, 25, 30]. These suggest that inflammation may be a future target of therapies and that intervention should begin even before the patients' initial hemodialysis session [34, 30.

In the next subsections, we provide information about the causes and consequences of inflammation and their influence to cardiovascular disease, the primary cause of mortality among ESRD patients undergoing HD .

\subsubsection{Causes of inflammation}

The causes of inflammation in ESRD patients undergoing hemodialysis are multifactorial. In the paper by Cobo et al. [35, the causes and consequences of inflammation on dialysis patients are outlined. One can see in figure 1.6 that the causes of inflammation are categorized according to whether they are related to impaired renal function, the dialysis technique, or comorbidities and lifestyle. Inflammation then leads to various other risk factors that contribute to the progression of PEW and atherosclerosis, and eventually death.

Reduced renal function causes retention of uremic substances and decreased clearance of cytokines, advanced glycation end products (AGEs), and prooxidants, which should normally be eliminated by the kidneys 25]. This uremic state serves as a stimulus that activates an inflammatory cascade by producing more proinflammatory immune cells and cytokines. Hypercytokinemia is a phenomenon observed in most ESRD patients [36]. The persistent production of cytokines promotes further inflammation. Furthermore, impairment or loss of renal function results in fluid overload. The presence of excess fluid directly affects the maintenance of blood pressure and its instability is detrimental to the heart. Congestive heart failure due to fluid overload is associated to the uremic inflammation experienced by ESRD patients 6.

Chronic inflammation in ESRD patients undergoing HD may also be caused by the dialysis procedure itself. Among the sources of this complication include incompatibility with the dialyzer membrane, impure dialysate, and vascular access infection [36, 37, 35]. Unstable blood pressure is common in dialysis patients and up to one-third of dialysis patients experience intradialytic hypotension (IDH) 38. One important goal of dialysis is to take out the excessive fluid that had accumulated in the body but at the same time, minimizing the occurrence of hypotension and hypovolemia. Achieving the socalled "dry weight", which is one's supposed weight when one's kidneys are functioning well, posts a challenge to the physicians since measuring blood volume is not simple and blood volume varies among different individuals $[22$. The rates of ultrafiltration and vascular refilling during hemodialysis play important roles in the maintenance of normal blood volume. Importance should also be given to the treatment time, interdialytic weight gain, as well as patient-specific factors such as inflammation, to ensure good 


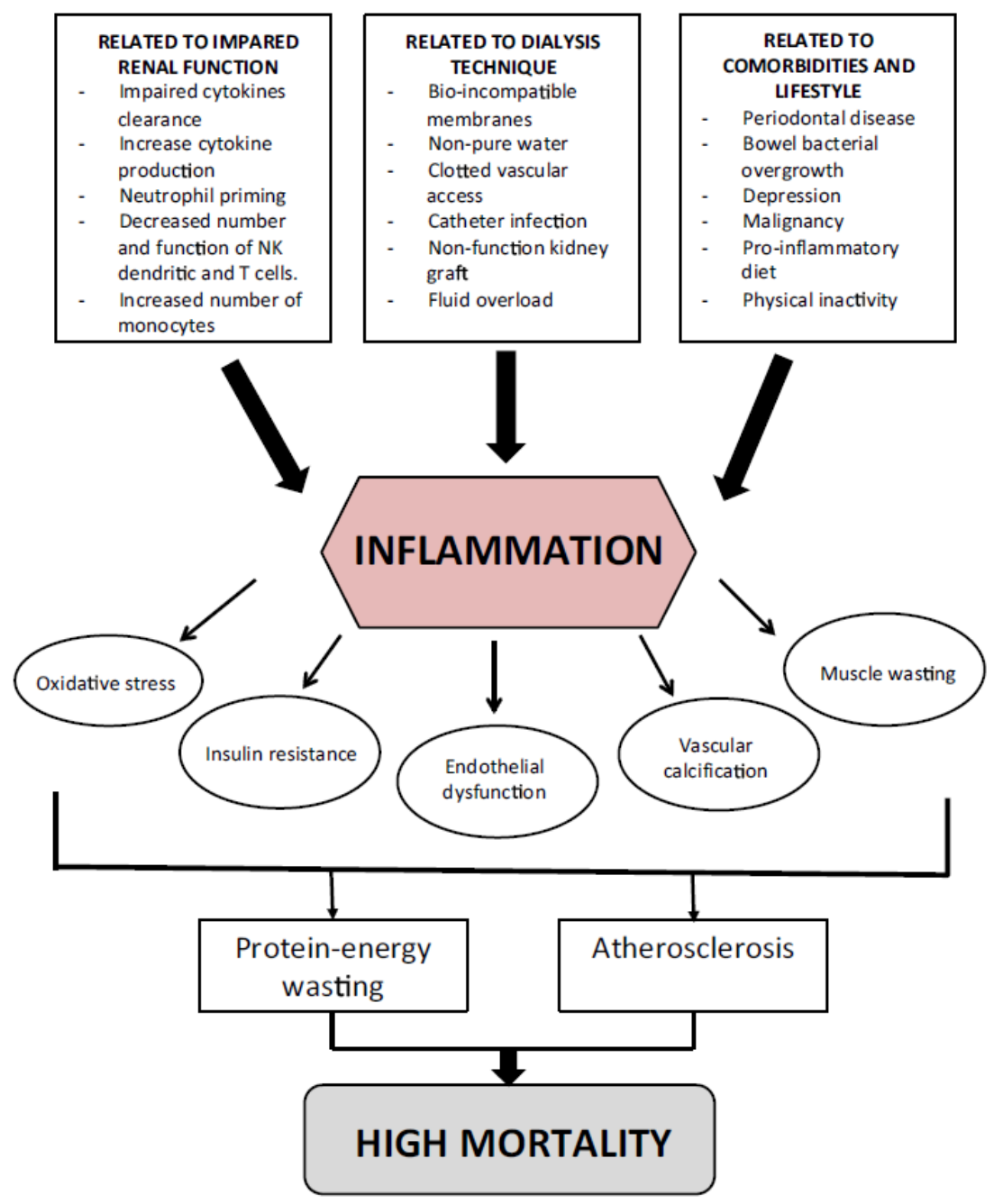

Figure 1.6: Causes and consequences of inflammation in dialysis patients. Reprinted from [35].

outcome of HD patients 38 .

Finally, inflammation may also be caused by comorbidities and lifestyle. Sedentary lifestyle, depression, and poor diet are all associated with promoting inflammation. Existence of other diseases or disorders such as periodontal disease and other persistent infections cause production of proinflammatory immune cells and cytokines.

Besides the causes already mentioned, there are still other inflammatory events, which vary among patients. These suggest that genetic factors may also play a role in the inflammatory response in HD patients. For example, there is great difference between what is meant by elevated levels of inflammatory markers among dialysis patients from different regions. In the study by Bazeley et al. 39 involving prevalent HD patients in 10 countries, they showed that the median CRP level of HD patients in Japan is 1.0 
$\mathrm{mg} / \mathrm{L}$, while in Europe, Australia and New Zealand it is $6.0 \mathrm{mg} / \mathrm{L}$. Also, occurrence of inflammation in Asian dialysis patients appear less likely than Caucasian dialysis patients and this translates to improved mortality rate for Asian dialysis patients [36]. Furthermore, studies have suggested that CRP and IL-10 production show substantial heritability and that genetic variations in individuals cause different responses to uremic inflammation [33].

\subsubsection{Inflammation as a cardiovascular risk factor}

The most common cause of death among ESRD patients undergoing hemodialysis is cardiovascular disease. The annual mortality rate of ESRD patients due to CVD is 10 to 20 times higher than the general population [34. Cardiovascular risk factors are already commonly observed in predialysis and incident dialysis patients. Signs of advanced atherosclerosis are seen in patients who had just started dialysis [40].

Inflammation plays an important role in the accelerated atherosclerosis and malnutrition that is common in ESRD patients undergoing HD. The coexistence of malnutrition, inflammation, and atherosclerosis is sometimes referred to in literature as the MIA syndrome [34. The MIA syndrome experienced by ESRD patients suggests that these risk factors are greatly interrelated, interacting with each other in a vicious cycle that is believed to be a cause of the high mortality in ESRD patients 34, 32. Therefore, addressing the MIA syndrome during treatment may improve a patient's chance of survival 36 .

Malnutrition and protein energy wasting (PEW) sometimes appear indistinguishable in literature. Protein energy wasting is related to loss of protein and energy fuel stores in the body, while malnutrition simply refers to inadequate nutrient uptake [32]. Protein energy wasting is associated with low albumin levels, loss of body weight and muscle mass. Serum albumin is a nutritional biomarker that has been used as an indicator of the nutritional state of a patient. Low serum albumin may mean that PEW is present and when measured together with other inflammatory markers, it may also suggest the presence of inflammation 28, 41. Hypoalbuminemia, which is common in ESRD patients, is a sign of malnutrition which contributes to PEW. Despite having therapies targeting nutritional intake, efforts had not been enough to improve outcome. It is believed that malnutrition and hypoalbuminemia are effects of inflammation [42]. High levels of proinflammatory cytokines enhance malnutrition and catabolism, and these promote PEW in ESRD patients [32]. Loss of muscle mass is correlated with high levels of C-reactive protein (CRP) and interleukin-6 (IL-6), while loss of appetite is associated with high levels of IL-6 and tumor necrosis factor $\alpha$ (TNF- $\alpha)$. Depression is associated with increased level of IL-6 and affects food intake [33]. Besides malnutrition, other factors adding to PEW in CKD patients include changes in hormones affecting appetite, hypercatabolism, and resistance to or inhibition of anabolic hormones such as 
insulin and insulin-like growth factor (IGF), testosterone, and growth hormone (GH) 32. Figure 1.7 outlines the different ways how inflammation influences malnutrition and increased catabolism, which are integral in promoting PEW in ESRD patients.

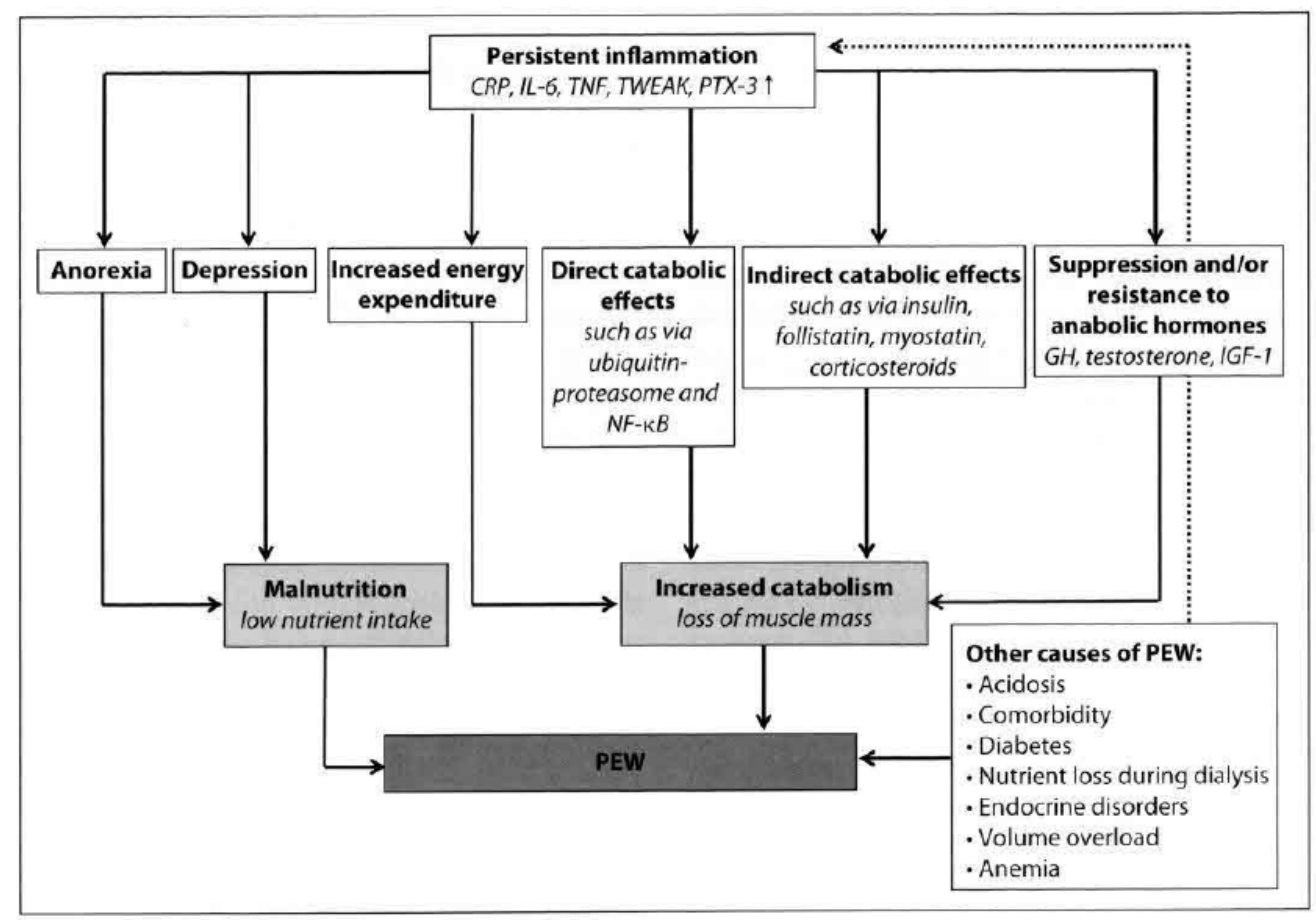

Figure 1.7: How inflammation affects PEW in ESRD patients. Adapted from 32.

It has been established that chronic inflammation plays a major role in the accelerated progression of atherosclerotic CVD in patients with ESRD [34, 27, 6. Acute phase reactants and proinflammatory cytokines have direct and indirect proatherogenic effects that may contribute to the accelerated atherosclerosis common in ESRD patients 11. Acute phase reactants may not only be markers of inflammation but may also be mediators to the initiation and progression of atherosclerosis. It was hypothesized that inflammatory stimuli cause changes on the endothelial wall and stimulate production of adhesion molecules, growth factors and chemokines [12]. There is also evidence that $\mathrm{CRP}$, fibrinogen, serum amyloid A (SAA), and Lp(a) have direct proatherogenic effects [43, 44, 45]. Elevated levels of IL-6 is associated with worsening early atherosclerosis in mice [46], poor cardiovascular outcome in elderly patients 47], and occurrence of myocardial infarction in healthy men 48 .

Inflammation may indirectly affect other cardiovascular risk factors such as vascular calcification, oxidative stress, and endothelial dysfunction. Vascular calcification is the build up of calcium on the walls of the blood vessels. It is more common in hemodialysis patients compared to the general population and is associated with cardiovascular atherosclerosis [37]. The proinflammatory cytokine TNF- $\alpha$ is said to enhance calcifi- 
cation. Calcification inhibitors such as fetuin-A appear to be down-regulated in the presence of inflammation [33. Furthermore, TNF- $\alpha$ is said to suppress the secretion of apolipoprotein E (Apo E), which is associated with the metabolism of intermediatedensity lipoprotein 36 and calcification of vascular cells 49,50 . Oxidative stress, which describes an imbalance between production of reactive oxygen species and the antioxidant defenses, is also common in ESRD patients. It is associated with endothelial dysfunction and believed to also be enhanced by inflammation 27. Both oxidative stress and endothelial dysfunction are known factors that contribute to atherosclerosis development. Malnutrition and increased proinflammatory cytokines are related to congestive heart failure (CHF) [51 in the general population, but further studies are needed to see whether this also holds for CKD and ESRD patients on HD [34.

\subsection{Biomarkers of inflammation}

\subsubsection{Inflammatory markers}

In the late 1990s, inflammation had been recognized as having a significant part in CKD and ESRD [52] and since then, numerous studies involving biochemical inflammatory markers have emerged. The circulating levels of proinflammatory markers are several times higher in CKD patients than in the general population [53, 31] and their levels increase as kidney function declines [54, 33]. In 2011, Miyamoto et al. [30] published in their paper a summary of the markers that had been related to cardiovascular risk or mortality prediction in patients with CKD. Figure 1.8 shows the list. Other studies on inflammatory markers $28,42,55,30$ have looked at acute phase reactants such as CRP, serum amyloid A (SAA), and fibrinogen; proinflammatory cytokines such as IL-6 , IL18 , IL-1 $\beta$, and TNF- $\alpha$; and adhesion molecules such as intracellular adhesion molecule-1 (ICAM-1) and vascular cell adhesion molecule-1 (VCAM-1). Pentraxin 3 (PTX3) and TNF-like weak inducer of apoptosis (TWEAK) are markers which are both associated with inflammation and PEW [32]. Decreased levels of albumin [32] or anti-inflammatory cytokines like IL-10 [31] are also indicators of activated inflammatory response. Some of these biomarkers not only serve as markers to detect presence of inflammation but may also be risk factors for vascular disease 33 .

Among the inflammatory biomarkers mentioned, CRP and IL-6 are the most widely studied. Both biomarkers have been shown to independently predict all-cause and cardiovascular mortality in ESRD patients [55, 12]. Currently, CRP is the most common and widely used prototypic marker of inflammation and predictor of cardiovascular risk. C-reactive protein is a reliable marker because it is stable, and independent of food intake and circadian variation [56, 35. It can be measured readily and inexpensively by blood testing. The development of high-sensitive CRP (hsCRP) assay is significant because it is able to measure very low levels of CRP and may help detect low grade 


\begin{tabular}{|c|c|}
\hline Markers & Prognostic value \\
\hline C-reactive protein & $\begin{array}{l}\text { Most widely used inflammatory marker predicting future cardiovascular risk and short-term mortality in various } \\
\text { stages of CKD }[3,6]\end{array}$ \\
\hline Interleukin-6 & Best inflammatory outcome predictor for total and cardiovascular mortality in various stages of CKD $[6,13]$ \\
\hline sTWEAK & Predictor of cardiovascular and all-cause mortality in nondialysis ESRD [14] and hemodialysis patients [15] \\
\hline STRAIL & Low sTRAIL level is a predictor of all-cause mortality in a patient group with CKD $1-5 D[16]$ \\
\hline Interleukin-18 & Predictor of future hospitalization events in hemodialysis patients [1] \\
\hline Pentraxin-3 & Predictor of cardiovascular and all-cause mortality in nondialysis ESRD $[14,17]$ and hemodialysis patients $\left[18^{\circ}\right]$ \\
\hline Endotoxin & Predictor of 1 -year all-cause mortality in CKD $3-5$ patients $\left[4^{\bullet \bullet}\right]$ \\
\hline White blood cells & Predictor of all-cause mortality in hemodialysis patients [1] \\
\hline Fibrinogen & Predictor of cardiovascular and all-cause mortality in hemodialysis patients [1] \\
\hline Hyaluronan & Predictor of all-cause mortality in nondialysis ESRD patients [1] \\
\hline Myeloperoxidase & Predictor of all-cause mortality in hemodialysis and peritoneal dialysis patients [1] \\
\hline S100A12 & Predictor of cardiovascular and all-cause mortality in hemodialysis patients [27] \\
\hline Gelsolin & Low gelsolin level is a predictor of 1 -year all-cause mortality in hemodialysis patients [29] \\
\hline
\end{tabular}

Figure 1.8: Inflammatory biomarkers associated with cardiovascular risk or mortality in CKD patients. Reprinted from [30].

inflammation [57, 35]. On the other hand, IL-6 is said to be the best inflammatory marker for classifying risk in CKD patients 33 and also the best predictor of total and cardiovascular mortality [30]. However, IL-6 testing is not commonly used in clinics [30]. Although the levels of CRP and IL-6 may have substantial variation, there is a strong evidence that the two markers are correlated [4].

It is evident that ESRD and inflammation are greatly interrelated and the causes and consequences of inflammation involve a multitude of mechanisms. Inflammatory biomarkers have been studied to evaluate risks for mortality and CVD. It was proposed in 10 that lowering the level of CRP and IL-6 through aspirin and statins may reduce the cardiovascular mortality in ESRD patients. Although this remains unverified, it is clear that inflammation is an obvious target of therapies [31]. Treatment strategies already suggested include determining the underlying cause of infection, lifestyle and diet changes, anti-inflammatory and anti-cytokine therapies 33, 56. Regular monitoring and multiple measurements of CRP also appear to be helpful in clinical decision-making, which can lead to better survival rate $35,53,33$.

At present, there is a challenge in determining the presence of inflammation in ESRD patients, assessing its level and risk, and suggesting ways how it can be addressed during HD therapy. There have been a lot of studies that associate biomarkers with an evident presence of inflammation and higher cardiovascular mortality. We are interested in the association of inflammation, via the measured level of an inflammatory biomarker, to changes on the capillary wall that may affect capillary refilling during HD. With the aid of a mathematical model, we would like to examine how the fluid status of a patient with inflammation changes during HD. With the knowledge of the possible changes inflammation has on capillary refilling may assist physicians in planning a HD treatment suited for an ESRD patient with inflammation. 


\subsubsection{The C-reactive protein}

C-reactive protein $(\mathrm{CRP})$ is an acute-phase response protein, a member of the pentraxin family that is produced by the liver, stored in the endoplasmic reticulum, and released when induced by proinflammatory cytokines such as IL-6, IL-1 and TNF [56]. It was discovered by Thomas Francis and William Tillet in 1930 as a substance that can be taken from patients infected with pneumococcus. They initially referred to it as "fraction C" 14. C-reactive protein can increase up to more than 500 times compared to normal levels, depending on the extent of inflammation, within 6-8 hours after a stimulus. It has a half-life of 19 hours and its clearance rate is almost constant in normal and inflamed individuals. This makes CRP easily detectable in blood [53 and a reliable marker of inflammation [58, 59].

C-reactive protein predicts cardiovascular mortality in the general population [28]. Furthermore, studies by [59, 60, 61 say that CRP is a good predictor of all-cause and cardiovascular mortality in ESRD patients. Activated acute-phase response, as evidenced by increased levels of CRP, appears to promote vascular disease which leads to a higher risk of death in HD patients [28, 12, 57]. This means that besides being a marker for inflammation, CRP seems to have an active role in vascular disease development, particularly accelerated atherosclerosis, that is common in ESRD patients [59, 56].

Evidence that CRP plays a direct role in vascular disease development such as atherosclerosis are enumerated in the following papers [59, 56, 4, 57]. Firstly, CRP affects the production of monocytes and macrophages, and recruitment of other inflammatory mediators. Secondly, CRP promotes endothelial dysfunction by stimulating expression of adhesion molecules. Thirdly, CRP promotes foam cell formation from low density lipoproteins, activates the complement system, and thus, it stimulates the formation of atherosclerotic lesions. Fourthly, CRP affects vascular smooth muscle (VSM) cells by stimulating the VSM migration and proliferation and production of reactive oxygen species (ROS). Finally, CRP also inhibits nitric oxide production, which induces endothelial cell apoptosis and inhibits angiogenesis, contributing to plaque formation. Although all these suggest that CRP may directly promote vascular disease, further studies in the ESRD or CKD setting are still needed 44.

It is known that declining renal function is associated with increasing levels of CRP. In this study, inflammation is characterized by an elevated level of CRP in plasma. It was estimated that between $30-50 \%$ of predialysis, HD and PD patients have CRP serum level of at least $8 \mathrm{mg} / L[10$. Centers for Disease Control and Prevention (CDC) and the American Heart Association (AHA) in 2003 released guidelines regarding the cardiovascular risk associated with the level of measured CRP for the general population. According to the guidelines, CRP level that is less than $1 \mathrm{mg} / \mathrm{L}$ means low risk, CRP level between 1 and $3 \mathrm{mg} / \mathrm{L}$ means medium risk, and CRP level that is greater than or equal to $3 \mathrm{mg} / \mathrm{L}$ means high risk. This was extended to very high risk when CRP level 
greater that $10 \mathrm{mg} / \mathrm{L}$ and very low risk when CRP level is less than $0.5 \mathrm{mg} / \mathrm{L}$. Using this standard, most if not all CKD and ESRD patients belong to high or very high risk.

Some of the methods to measure CRP are enzyme-linked immunosorbent assay (ELISA), immunoturbidimetric, and immunonephelometric assays, which utilize color change or light to measure CRP concentration. The conventional assays have detection limit of at least $3 \mathrm{mg} / \mathrm{L}$. High-sensitivity CRP assays were more recently developed, which can detect CRP level of $0.007 \mathrm{mg} / \mathrm{L}$ [57]. According to Stenvinkel [4], the median $\mathrm{CRP}$ level of the general population is $1.5 \mathrm{mg} / \mathrm{L}$. Also, CRP levels of perfectly healthy young persons can be as low as $0.15 \mathrm{mg} / \mathrm{L}$ [33]. According to Carrero and Stenvinkel in 2010 [33, dialysis patients in Europe have an average CRP level of 7-8 $\mathrm{mg} / \mathrm{L}$. US dialysis patients have higher CRP levels, while Japan and Korea dialysis patients have lower CRP levels, compared to that of European dialysis patients. Furthermore, dialysis patients with diabetes have higher CRP levels compared to those without diabetes [33]. At present, there are no guidelines on risk stratification based on CRP levels similar to the one by CDC and AHA that is appropriate for CKD and ESRD patients [56, 53]. Adding to this shortcoming are considerable variations in CRP levels that exist among different race and among patients with comorbidities.

A definition of "high" CRP level also vary among studies in the available literature. In [35, 53, it was suggested that a CRP level of at least $10 \mathrm{mg} / L$ is associated with uremic inflammation. Cut-off levels for high CRP are set as $5 \mathrm{mg} / \mathrm{L}$ in 62,63 ; 8 $\mathrm{mg} / \mathrm{L}$ in 28]; and $10 \mathrm{mg} / \mathrm{L}$ in 40,64 . An appropriate cut off level for high CRP that is applicable to ESRD patients on hemodialysis still needs to be developed [53] and treatment strategies addressing increased CRP levels needs to be studied [35].

Since CRP is a nonspecific marker of inflammation, its clinical contribution has been ignored for years. Only when studies regarding CRP and its association with cardiovascular disease had emerged that CRP gained importance in research [14. The nonspecificity of CRP may be regarded as a disadvantage because it does not provide physicians about the source of inflammation. On the other hand, we know that chronic inflammation in HD patients is a combined effect of a number of malfunctioning processes. Hence, pinpointing the specific sources of inflammation may not be any more relevant, if not impossible. Another issue about CRP is that it is variable and fluctuating 35. This means that a single CRP measurement may not accurately reflect the inflammatory state or predict outcome of a patient. To address this, studies such as that of Meuwese (2011) 53] suggest regular monitoring instead of single CRP measurement. Despite its drawbacks, the number of clinics using CRP testing has increased in ten years [35. Some advantages of including CRP test in the HD routine are early prognosis of CV risk, prevention of possible sources of infection, and awareness that leads to compliance in therapies and improvement in diet and lifestyle. With the information 
provided by regular CRP monitoring, physicians may be guided accordingly and may eventually improve outcome in ESRD and CKD patients [53].

\subsection{Effects of inflammation on the capillary wall}

The arterioles, capillaries, and venules, which comprise the microcirculation, have diverse chemical and physical responses during inflammation. The inflammatory response of the microcirculation aims to locally deliver inflammatory cells to the site of inflammation, isolate unaffected tissue, and initiate tissue repair [65]. Inflammation has the following effects: impairs the reaction of vasodilators especially in the arterioles, enhances adhesion of leukocytes and platelets to endothelial walls especially in the venules, increases endothelial cell migration and proliferation that is responsible for angiogenesis that prolong and intensify inflammation, reduces capillary perfusion due to leukocyte plugging, reduces delivery of blood to tissues in the long-term, and promotes pro-thrombotic and pro-coagulant effects that may contribute to further inflammation [65]. These occur through various physical and chemical responses affecting the endothelial cells, vascular smooth muscle cells, leukocytes, platelets, and other mediators.

For instance, the normal response of endothelial cells to vasodilators is to produce nitrogen oxide (NO) that causes dilation and relaxation of vascular smooth muscles, especially in the arterioles. During inflammation, this response is impaired because various inflammatory responses promote production of reactive oxygen species (ROS) and superoxide, which cause constriction of vascular smooth muscles and inhibition of NO production. The slow movement of leukocytes in the capillaries may lead to capillary plugging and decreased capillary perfusion. Furthermore, activation of ROS and superoxide enhances the adhesion of molecules and degradation of the extracellular matrix of the vessel walls, which in the long-term may lead to endothelial dysfunction [65]. Degradation of the extracellular matrix also makes the interstitium more compliant. This means that a significant increase in interstitial volume causes only a small increase in interstitial hydrostatic pressure 66. Through these processes, we see that inflammation impairs the normal responses affecting hydrostatic pressure changes, fluid flux, and lymph flow.

Inflammation may also change the ultrastructure of the endothelial wall, increase the recruitment of capillaries, and increase diffusive and convective exchange of fluid and proteins between the vascular and interstitial spaces. Inflammatory responses that are greatly important to this study are related to the effects on the endothelial wall. In many studies 67, 68, 69, 70,71, inflammation is characterized by increased vascular permeability to fluid and macromolecules. A number of experiments on acute inflammation applied to animal tissues demonstrated increased permeability to fluid and molecules through opening of gaps in the venules and capillaries [69]. It is noteworthy that al- 
though capillaries are the major site of fluid leakage, most protein leakage occur at the interendothelial junctions in the postcapillary venules [65, 70]. Nevertheless, the fluid and protein leakage results in considerable changes in the Starling forces that affects vascular refilling.

According to 72 , permeability describes the ability of a capillary membrane to restrain the flow of fluid and macromolecules that are driven by a physical force. It is quantified through parameters such as hydraulic conductivity, solute permeability, and oncotic reflection coefficient. The increase in vascular permeability accompanying inflammation is naturally attributed to an increase in filtration coefficient $L_{p}$ or a decrease in osmotic reflection coefficient $\sigma$. An increase in $L_{p}$ not only means increased fluid flow to the interstitial space, but also increased transfer of proteins that comes with the fluid. In this way, interstitial oncotic pressure may increase. Inflammation also promotes recruitment of capillaries, which means that more capillaries are opened by precapillary sphincters to blood flow. This increases the surface area available for exchange and hence, also increases $L_{p}$ 66. Meanwhile, a decrease in $\sigma$ enables more macromolecules to pass through the membrane and this reduces the oncotic pressure gradient between the plasma and interstitial spaces 66. Referring to equation (1.1), decreasing $\sigma$ and oncotic pressure gradient $\triangle \pi=\pi_{p}-\pi_{i}$ cause the fluid flux $J_{v}$ to decrease. Meanwhile, increasing the filtration coefficient $L_{p}$ increases $J_{v}$. Changes in $J_{v}$ affects interstitial and plasma volumes, protein concentrations, hydrostatic pressures, and lymph flow.

\subsection{Goal of the study}

Recently, de los Reyes et al. published a study 13] describing the fluid dynamics in HD patients during the initial hour of treatment. The study considered two compartments, the plasma and interstitial compartments, separated by a semi-permeable membrane. In each compartment, the volume of fluid and the concentration of proteins are monitored. Vascular refilling from the interstitial space and lymph is observed as ultrafiltration is initiated.

This study extends the model by 13 to examine how systemic inflammation, which is commonly suffered by HD patients, affects fluid dynamics during HD. Numerous studies have established that inflammation is experienced by HD patients. We propose a model to provide an insight of how inflammation may affect fluid movement in HD patients during ultrafiltration. We assume that inflammation has an influence on the capillary wall. As a consequence of the changes on the capillary wall, capillary refilling is affected. We quantify inflammation based on the concentration of the inflammatory biomarker C-reactive protein (CRP). The association of inflammation via CRP level to the capillary wall properties $L_{p}$ and $\sigma$ are examined and functions describing these are formulated. The formulated functions are built in to the model of vascular refilling 
(13) presented earlier in this chapter. Using the model, fluid and protein concentration dynamics for different CRP levels during the initial hour of HD treatment are observed. The additional parameters appearing in the formulated functions are analyzed, using hematocrit and fluid flux as model outputs. Sensitivity analysis, parameter estimation using least squares, and subset selection based on asymptotic standard errors are used to study the additional model parameters. 



\section{Chapter 2}

\section{Mathematical model}

In this chapter, we propose a model that describes vascular refilling during ultrafiltration of hemodialysis patients with inflammation. We incorporate inflammation to an earlier model by de los Reyes et. al [13, which describes the fluid dynamics of patients undergoing HD during ultrafiltration for one hour after initiation of the treatment. During this period, it is assumed that vascular refilling from the lymphatic system is constant. Hence, changes in fluid status of the vascular and interstitial spaces are highly dependent on the refilling through the capillaries. With the presence of inflammation, capillary refilling may be perturbed. It is natural to look at how the refilling process might be affected by changes on the capillary wall properties, such as filtration coefficient $L_{p}$ and reflection coefficient $\sigma$. We characterize inflammation based on the elevated levels of the inflammatory marker CRP. We present a quantitative way to describe the relationship of CRP, $L_{p}$, and $\sigma$ by formulating functions in terms of the CRP concentration in the plasma. Then we incorporate these functions to the vascular refilling model by 13 that is presented in the previous chapter. Finally, we look at some simulations of the fluid and protein concentration dynamics in the plasma and interstitial spaces as CRP concentration increases.

We begin with a discussion of the available data for the inflammatory biomarker CRP. In this study, the level of CRP will be used as the indicator of the presence and severity of inflammation in HD patients. Data for CRP are taken from published studies, where the participants in the study group are patients on dialysis, without particular consideration to the presence or absence of comorbidities. Next, we discuss available data for the capillary filtration coefficient $L_{p}$ and osmotic reflection coefficient $\sigma$. Functions describing the relationship of CRP to $L_{p}$ and $\sigma$ are then formulated under some assumptions. 


\subsection{Data for CRP levels in HD patients}

There are numerous studies which give information about CRP levels among HD patients. In these studies, the data provided are usually in terms of CRP median and interquartile range (IQR), or CRP mean and standard deviation. There are two main references for CRP data which are used in this study. These are by Kaysen et al. [73] in 2000 and by Meuwese et al. [74 in 2011. These references were chosen mainly because in the method of their study, they considered participants that represent a mixed dialysis patient population and the data are given in terms of median and IQR. The study population did not specifically consist of patients with or without certain conditions such as diabetes, albuminuria, existing cardiovascular disease, high or low concentration of inflammatory markers, high body mass index (BMI), and so on.

In the first reference [73], the patient group consisted of 37 patients who are enrolled in the Hemodialysis (HEMO) study. CRP, other acute-phase proteins, and albumin, were measured weekly before dialysis for a period of six weeks. After that, monthly measurements were made. Serum CRP was measured with rate nephelometry using a Beckman Array automated nephelometer. The HEMO study protocol is described more in detail in 75, 76. In the second reference [74, they looked at two independent groups of patients and so, there are also two sets of CRP data given. The first group consisted of 201 prevalent patients on maintenance HD belonging to the Mapping of Inflammatory Markers in Chronic Kidney Disease (MIMICK) cohort 77. Serum CRP was measured twice, first at the beginning of the trial and the second time after 3 months. They measured serum CRP using an immunometric assay called Immulite Analyser (Immulite, DPC, Siemens, CA) with a detection limit of $0.1 \mathrm{mg} / \mathrm{L}$. The second group of patients consisted of 472 incident dialysis patients from Netherlands Cooperative Study in the Adequacy of Dialysis (NECOSAD) cohort [78]. CRP was measured with an immunoturbidimetric assay with detection limit of $3 \mathrm{mg} / \mathrm{L}$. The serum CRP measurements were done at the third and sixth months. Table 2.1 shows the median and interquartile range (IQR) values of the measured CRP values that we obtained from the mentioned references.

\begin{tabular}{|r|c|c|c|}
\hline \multicolumn{2}{|c|}{ reference } & median CRP & quartiles 1 to 3 \\
\hline \hline 74 & 73 & 9.91 & $3.64-20.5$ \\
74 & MIMICK & 6.4 & $2.6-18.5$ \\
74 & NECOSAD & 6.0 & $3.0-16.0$ \\
\hline
\end{tabular}

Table 2.1: Summary of CRP data, units in $\frac{m g}{L}$

Other studies which measured CRP values among hemodialysis patients are 55,10, 28, 40, 79, 63, 64, 80. For instance, CRP median and IQR were $7.5 \mathrm{mg} / \mathrm{L}$ and 3.4-16.7 
$m g / L$, respectively, from 217 ESRD patients on regular hemodialysis and peritoneal dialysis [55]. In [10, 40, 28], CRP levels were measured and compared against risks for cardiovascular disease, malnutrition, and atherosclerosis among dialysis and predialysis patients. In [63], CRP level and mortality among Brazilian HD patients were studied. In [64], the study compares CRP levels in 3-month intervals and investigated how the changes in concentration are related to mortality. It concluded that the groups with high concentration of CRP in at least one occasion have an increased mortality risk than those with consistently low CRP concentration. The studies $[79,80$ looked at CRP levels before and after a hemodialysis session to see if intradialytic factors cause fluctuating CRP levels or not. They also determined how the fluctuations are associated with mortality risk.

Regarding as to whether CRP levels change during a single HD session, varying results exist. For example, a study in 2004 showed that $25 \%$ of the patients had an increase of at least $0.5 \mathrm{mg} / L$ in CRP levels after a hemodialysis session [79]. The same study also showed that the CRP level returned to the initial level before the next dialysis session and concluded that an increase in CRP level during an HD session is associated independently with increased mortality. On the other hand, a study in 2010 involving MIMICK and NECOSAD patient groups showed that there is no change in CRP levels and that there is no association between changes in CRP levels in a single dialysis session with mortality [80]. These studies show that the CRP level among dialysis patients may change over a time scale of weeks or months, which makes sense since chronic inflammation also progresses gradually.

\subsection{Data for filtration and reflection coefficients}

Studies providing data for the filtration coefficient $L_{p}$ among hemodialysis patients are limited. Schneditz et al. in 1992 [81] developed an open, two-compartment model describing the dynamics of blood and plasma volume during UF and refilling within the first hour of HD. Using Starling's equation, they were able to calculate for $L_{p}$ given the measurements for hematocrit, plasma protein concentration, plasma density and blood density. In the results, they obtained calculated and fitted $L_{p}$ values for 13 cardiovascularly stable HD patients. The mean calculated $L_{p}$ is $5.6 \mathrm{~mL} / \mathrm{mmHg} / \mathrm{min}$ and the values ranged from 3.2 to $8.0 \mathrm{~mL} / \mathrm{mmHg} / \mathrm{min}$.

In a study by Yashiro et al. in 2002 [82], $L_{p}$ of 21 patients on HD, excluding those who are overhydrated, varied between 1.4 and $8.4 \mathrm{~mL} / \mathrm{mmHg} / \mathrm{min}$. In another study by Yashiro et al. in 2005 [83, they illustrated that the mean $L_{p}$ can be as low as $0.83 \mathrm{~mL} / \mathrm{mmHg} / \mathrm{min}$ and can reach up to around $10 \mathrm{~mL} / \mathrm{mmHg} / \mathrm{min}$. In both studies, the kinetic model by Schneditz et al. 81 was used to analyze the dynamics of plasma volume and hematocrit, and $L_{p}$ was determined by fitting the model to the 
hematocrit data.

In the vascular refilling model by de los Reyes et al. [13], the calculated $L_{p}$ values for 12 patients were given and they ranged from 2.071 to $30.3 \mathrm{~mL} / \mathrm{mmHg} / \mathrm{min}$. The $L_{p}$ value equal to $30.3 \mathrm{~mL} / \mathrm{mmHg} / \mathrm{min}$ seems exceptional when compared with the calculated $L_{p}$ values in the previously cited studies $81,82,83$. The very high value of $L_{p}$ is plausible; in fact, $L_{p}$ may be up to $32.7 \mathrm{~mL} / \mathrm{mm} \mathrm{Hg} / \mathrm{min}$ according to [13. This may be interpreted as the patient being fluid overloaded and/or inflamed [13].

The two main references for the values of $L_{p}$ which are used in this study are by 13 and [81]. In both cases, calculated $L_{p}$ values from HD patients were enumerated. From these values, we compute for the median and IQR of the sets of data. The computed values will later be compared with the median and IQR of the CRP data. Table 2.2 summarizes these information.

\begin{tabular}{|c|c|c|c|c|}
\hline reference & median $L_{p}$ & quartiles 1 to 3 & $\operatorname{minimum} L_{p}$ & $\operatorname{maximum} L_{p}$ \\
\hline |13| & 5.064 & $3.227-6.315$ & 2.071 & 30.3 \\
\hline 81 & 5.5 & $4.35-6.85$ & 3.2 & 8.0 \\
\hline
\end{tabular}

Table 2.2: Summary of $L_{p}$ data, units in $\frac{m L}{m m H g \cdot m i n}$

Data regarding the reflection coefficient $\sigma$ in hemodialysis patients are even more limited. In this study, we are considering the systemic reflection coefficient, which represents the average value of $\sigma$ in most capillary membranes with respect to most macromolecules.

Many studies on inflammation agree that inflammation causes increased vascular permeability, which translates to increased filtration coefficient. Cotran and Majno 84 observed that both venules and capillaries are affected during the delayed-prolonged inflammatory response in rats. Unlike the early-transient response, which is immediate and lasts only for some minutes, delayed-prolonged response occurs at a later time and lasts for several hours following the injury. Similarly, a study by Clough 68] on frogs suggested that the increased leakage might be due to formation of gaps, which decreases the value of the term $\sigma \Delta \pi$. When there is change in the ultrastructure of the vessel wall, then the coefficient $\sigma$ may decrease. Other studies on intestine, lung, hindpaw, and colon of cats, dogs, and sheep stated that the value of $\sigma$ for total plasma proteins is between 0.7-0.95 18 .

More useful data for this study are in [16, 85, 19]. According to [16], the value of $\sigma$ for most continuous and fenestrated capillaries is 0.8 to 0.95 . From 85], the systemic $\sigma$ to plasma albumin is $0.75-0.95$. A model by Levick in 2010 [19 simulated the increased permeability due to mild inflammation by increasing the number of large pores and 
fixing the number of small pores. It was showed that $L_{p}$ increased about $50 \%$ and $\sigma$ for plasma albumin decreased from 0.93 to 0.66 , which reflects mild inflammation.

\subsection{Formulating functions for filtration and reflection co- efficients}

In this part, the goal is to formulate functions that describe the relationship of the filtration coefficient $L_{p}$ and reflection coefficient $\sigma$ to the concentration of CRP. The idea is to use the average of the medians, first and third quartiles of CRP, $L_{p}$, and the mean $\sigma$ as points. We also set suitable upper and lower bounds for the parameters, if necessary. Fitting by least squares is used to determine appropriate coefficients for the formulated functions.

As mentioned from the previous section, the highest values for $L_{p}$ obtained in 81 and 82 are 8.0 and $8.4 \mathrm{~mL} / \mathrm{mmHg} / \mathrm{min}$, respectively. The highest $L_{p}$ value equal to $30.3 \mathrm{~mL} / \mathrm{mmHg} / \mathrm{min}$ obtained in 13 is regarded as the patient having severe fluid overload or inflammation. The next highest value in the data is $11.53 \mathrm{~mL} / \mathrm{mmHg} / \mathrm{min}$. At the moment, it seems reasonable to set the mean upper limit of $L_{p}$ to be $12.0 \mathrm{~mL} / \mathrm{mm} \mathrm{Hg} / \mathrm{min}$ and the mean lower limit to be $0 \mathrm{~mL} / \mathrm{mmHg} / \mathrm{min}$.

To have an idea for the average upper limit of CRP concentration, we refer to some studies that considered patients with high levels of CRP. In [53], the median and first to third quartiles for patients having stable high CRP levels are $29 \mathrm{mg} / \mathrm{L}$ and $22-$ $47.5 \mathrm{mg} / \mathrm{L}$, respectively for MIMICK group. In the same study but for the NECOSAD group, the median is $24 \mathrm{mg} / \mathrm{L}$ and the first to third quartiles are $17-48 \mathrm{mg} / \mathrm{L}$. In [64], the mean value for high $\mathrm{CRP}$ is about $35 \mathrm{mg} / \mathrm{L}$. Based on these, we set the upper limit for CRP concentration to be $50 \mathrm{mg} / \mathrm{L}$. But note that CRP levels can be much higher than this value, as in the case when there is infection.

In formulating a function for $L_{p}$ with respect to CRP concentration, we have the following assumptions:

(i) The function $L_{p}([C R P])$ is a general sigmoid function of the form

$$
L_{p}([C R P])=a+\frac{L}{1+e^{-d[C R P]}}
$$

where $a, L$ and $d$ are constants to be determined, and $[C R P]$ denotes the concentration of CRP in $m g / m L$. The parameter $d$ describes the growth rate, $a$ is the lower asymptote, and $L+a$ is the upper asymptote.

(ii) The graph of equation (2.1) is fitted to the points $(0.0074367,5.282),(0.00308,3.7885)$, and $(0.01833,6.5825)$. These three points correspond to the average of the medians and quartiles of CRP and $L_{p}$ given in tables 2.1 and 2.2. The data points are summarized in table 2.3 . 


\begin{tabular}{|c|c|c|c|}
\hline parameter & median & quartiles 1 to 3 & units \\
\hline \hline$L_{p}$ & 5.282 & $3.7885-6.5825$ & $\frac{m L}{m m H g \cdot m i n}$ \\
$\mathrm{CRP}$ & $7.4367 \times 10^{-3}$ & $3.08 \times 10^{-3}-18.33 \times 10^{-3}$ & $\frac{m g}{m L}$ \\
\hline
\end{tabular}

Table 2.3: Values used for $L_{p}$ and CRP

(iii) The graph of equation (2.1) is also fitted to the minimum CRP and $L_{p}$ values, which are assumed to be both 0 ; and to the maximum CRP and $L_{p}$ values, which are $0.05 \mathrm{mg} / \mathrm{mL}$ and $12 \mathrm{~mL} / \mathrm{mmHg} / \mathrm{min}$, respectively.

The parameters $a, L$, and $d$ appearing in equation (2.1) are calculated using fminsearch in Matlab; minimizing the sum of squares of the differences between the function value and $L_{p}$ value in table 2.3 . The formulated function associating CRP concentration with $L_{p}$ is given by

$$
L_{p}([C R P])=a+\frac{L}{1+e^{-d[C R P]}}
$$

where $a=-9.70, L=22.35$ and $d=66.23$. Figure 2.1 shows the graph of 2.2 for non-negative values of CRP concentration.

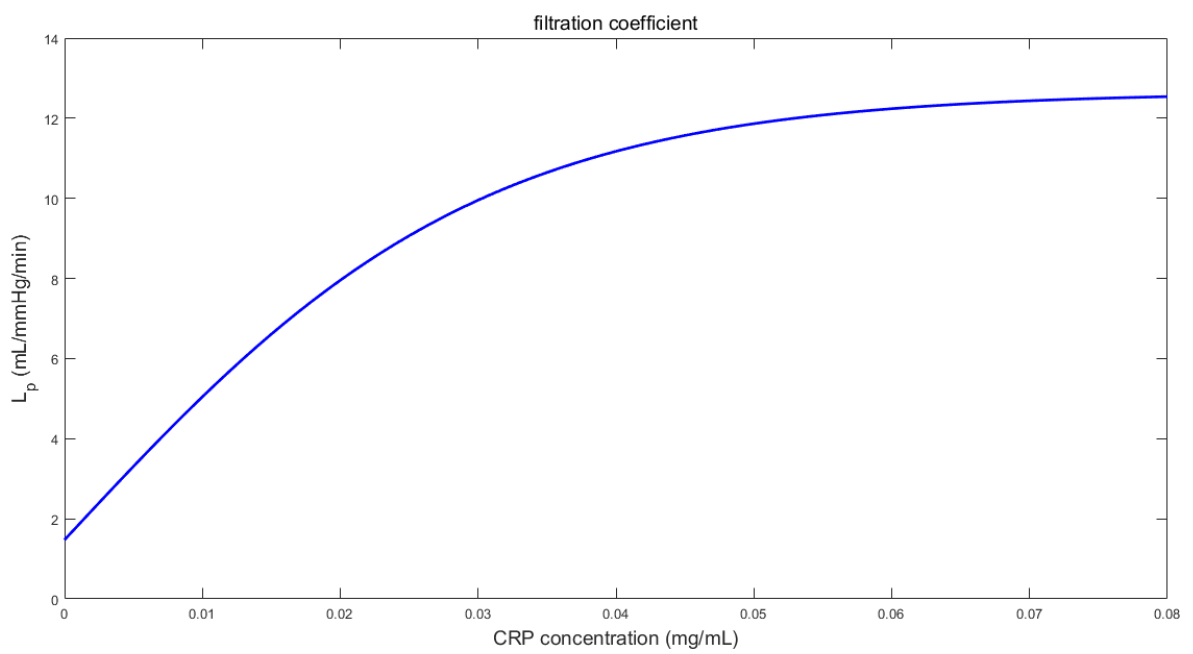

Figure 2.1: Graph of the function 2.2 with $a=-9.70, L=22.35$ and $d=66.23$.

Next, we do a similar formulation to describe the reflection coefficient $\sigma$ with respect to the CRP concentration. For this part, we have the following assumptions:

(iv) As CRP concentration increases, the value of $\sigma$ decreases. We assume that their relationship is of the form

$$
\sigma([C R P])=b e^{-\left(\frac{[C R P]}{c}\right)^{2}}
$$


where $b$ and $c$ are constants to be determined, and $0 \leq b \leq 1$.

$(v)$ The same median and IQR values for CRP in table 2.3 are used. We choose the range of values for systemic $\sigma$ to be $0.6-0.95$ based on $85,19,16$. So the graph of (2.3) is fitted to the points $(0.00308,0.95),(0.0074367,0.775)$, and $(0.01833,0.6)$ as we see in table 2.4 .

\begin{tabular}{|c|c|c|c|}
\hline parameter & mean/median & range/quartiles 1 to 3 & units \\
\hline \hline$\sigma$ & 0.775 & $0.6-0.95$ & dimensionless \\
CRP & $7.4367 \times 10^{-3}$ & $3.08 \times 10^{-3}-18.33 \times 10^{-3}$ & $\frac{m g}{m L}$ \\
\hline
\end{tabular}

Table 2.4: Values used for $\sigma$ and CRP

(vi) The value of $\sigma$ must lie between 0 and 1 . From equation (2.3), as long as $0 \leq b \leq 1$, then this requirement is satisfied.

In the same way, fminsearch is used to determine $b$ and $c$ by minimizing the sum of squares of the differences between $\sigma([C R P])$ and the $\sigma$ values in table 2.4. An equation relating $\mathrm{CRP}$ values and $\sigma$ is given by

$$
\sigma([C R P])=b e^{-\left(\frac{[C R P]}{c}\right)^{2}}
$$

where $b=0.9058$ and $c=0.0278$. The graph of 2.4 is shown in figure 2.2 for nonnegative values of CRP level.

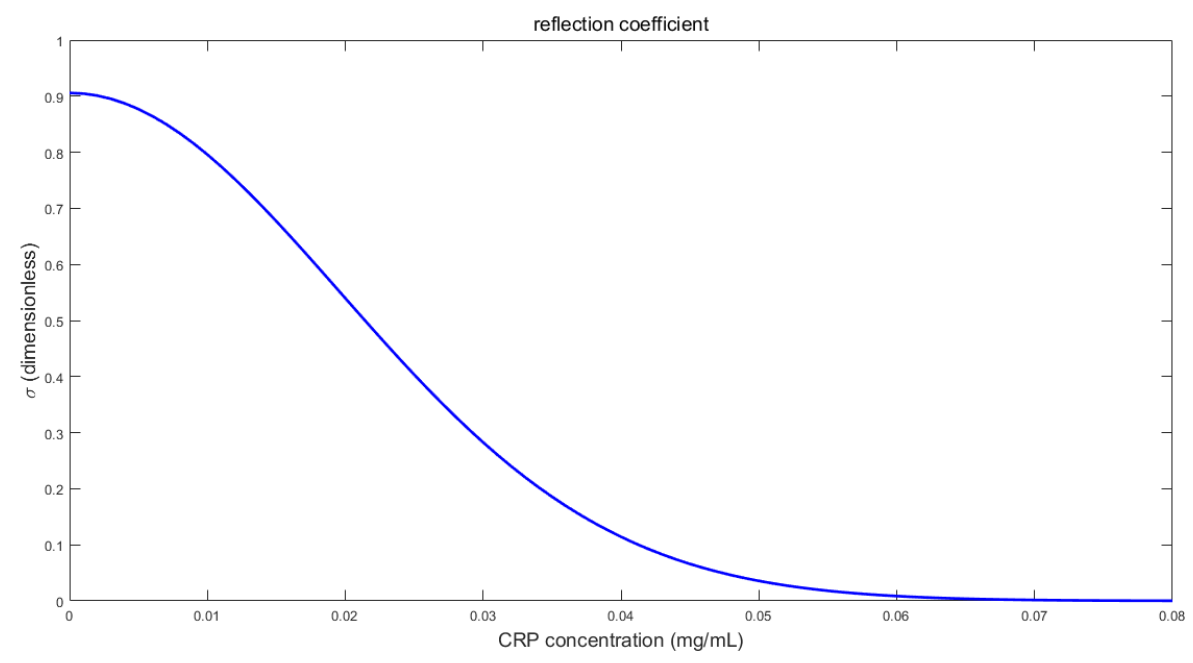

Figure 2.2: Graph of the function 2.4 with $b=0.9058$ and $c=0.0278$. 


\subsection{A model of vascular refilling with inflammation}

We now incorporate the functions $L_{p}([C R P])$ and $\sigma([C R P])$ that we have formulated to the vascular refilling model $(1.2)$ by de los Reyes et al. The proposed model aims to provide a link between inflammation and capillary refilling during the first hour of a hemodialysis treatment. In this study, the CRP concentration is our measure for the presence and severity of systemic inflammation. We interpret higher CRP levels as states having greater inflammatory condition.

The proposed model describing vascular refilling for HD patients with inflammation is presented in 2.5. Refer to table 2.5 for the list of parameters appearing in the model.

$$
\begin{aligned}
\frac{d V_{p}}{d t} & =J_{v}+\kappa-J_{U F} \\
\frac{d c_{p}}{d t} & =\frac{J_{s}-c_{p}\left(J_{v}+\kappa-J_{U F}\right)}{V_{p}} \\
\frac{d V_{i}}{d t} & =-J_{v}-\kappa \\
\frac{d c_{i}}{d t} & =\frac{-J_{s}+c_{i}\left(J_{v}+\kappa\right)}{V_{i}}
\end{aligned}
$$

where

$$
\begin{aligned}
J_{v}(t) & =L_{p}([C R P])\left\{\sigma([C R P])\left(\pi_{p}(t)-\pi_{i}(t)\right)-\left(P_{c}-P_{i}\right)\right\} \\
L_{p}([C R P]) & =a+\frac{L}{1+e^{-d[C R P]}} \\
\sigma([C R P]) & =b e^{-\left(\frac{[C R P]}{c}\right)^{2}} \\
\pi_{p}(t) & =a_{p, 1} c_{p}(t)+a_{p, 2} c_{p}(t)^{2} \\
\pi_{i}(t) & =a_{i, 1} c_{i}(t)+a_{i, 2} c_{i}(t)^{2} \\
J_{s}(t) & = \begin{cases}J_{v}(t)(1-\sigma([C R P]))\left(c_{i}(t)-\frac{c_{p}(t)-c_{i}(t)}{e^{x(t)}-1}\right)+\alpha \kappa, & J_{v}(t)>0 \\
-P_{s}\left(c_{p}(t)-c_{i}(t)\right)+\alpha \kappa, & J_{v}(t)=0 \\
J_{v}(t)(1-\sigma([C R P]))\left(c_{p}(t)-\frac{c_{p}(t)-c_{i}(t)}{e^{x(t)}-1}\right)+\alpha \kappa, & \\
J_{v}(t)<0\end{cases} \\
x(t) & =\frac{J_{v}(t)(1-\sigma([C R P]))}{P_{s}}
\end{aligned}
$$

The model has two equilibrium points $\left(V_{p}^{*}, c_{p, 1}^{*}, V_{i}^{*}, c_{i, 1}^{*}\right)$ and $\left(V_{p}^{*}, c_{p, 2}^{*}, V_{i}^{*}, c_{i, 2}^{*}\right)$. The calculations are shown in appendix $\mathrm{A}$. 


\begin{tabular}{|c|c|c|}
\hline parameter & meaning & unit \\
\hline$P_{c}$ & capillary hydrostatic pressure & $m m H g$ \\
\hline$P_{i}$ & interstitial hydrostatic pressure & $m m H g$ \\
\hline$P_{S}$ & permeability surface area product & $m L / \min$ \\
\hline$a_{p, 1}$ & coefficient of $c_{p}$ in $(2.7)$ & $m m H g(m L / m g)$ \\
\hline$a_{p, 2}$ & coefficient of $c_{p}^{2}$ in 2.7 & $m m H g(m L / m g)^{2}$ \\
\hline$a_{i, 1}$ & coefficient of $c_{i}$ in 2.8 & $m m H g(m L / m g)$ \\
\hline$a_{i, 2}$ & coefficient of $c_{i}^{2}$ in 2.8 & $m m H g(m L / m g)^{2}$ \\
\hline$\kappa$ & lymph flow & $m L / \min$ \\
\hline$\alpha$ & protein concentration in lymphatic fluid & $m g / m L$ \\
\hline$J_{U F}$ & ultrafiltration rate & $m L / \min$ \\
\hline$p_{\text {total }}$ & total protein mass in the system & $m g$ \\
\hline$[C R P]$ & concentration of CRP & $m g / m L$ \\
\hline$a$ & lower asymptote of $L_{p}$ in 2.2 & $\frac{m L}{m m H g \cdot \min }$ \\
\hline$L$ & related to the upper asymptote of $L_{p}$ in 2.2 & $\frac{m L}{m m H g \cdot \min }$ \\
\hline$d$ & growth rate in $(2.2)$ & $\frac{m L}{m g}$ \\
\hline$b$ & maximum value of $\sigma$ in 2.4 & dimensionless \\
\hline$c$ & constant appearing in $\longdiv { 2 . 4 }$ & $\frac{m L}{m q}$ \\
\hline
\end{tabular}

Table 2.5: List of parameters of the model

\subsection{Numerical simulations}

Tables 2.6 and 2.7 show the values of all parameters and initial conditions, used in the simulations in 13 . Table 2.8 gives the values of the additional parameters, which were introduced in the formulated functions for $L_{p}$ and $\sigma$. The simulations in this section, as well as those in the next chapter, use the values presented in tables 2.6 and 2.8. We set these values as the nominal parameter values. The nominal values, which we will see in the next chapter, are needed in the parameter estimation method.

First, we determine the CRP concentration that corresponds to the values listed in table 2.7. $L_{p}=1.65$ and $\sigma=0.9$. This is to establish the relationship of $[C R P], L_{p}$, and $\sigma$ and reproduce the dynamics of the states as presented in [13]. Then we look at what happens to $L_{p}$ and $\sigma$ as we increase the level of CRP, and observe the corresponding changes in fluid volume and protein concentration in the compartments. The numerical simulations are done using ode15s in Matlab.

When CRP concentration is equal to $0.45 \times 10^{-3} \mathrm{mg} / \mathrm{mL}$, we get $\sigma=0.9056$ and $L_{p}=1.6415$, which are relatively close to the values given in table 2.7. This CRP level is considered low for the general population and for patients on HD. This may be interpreted as an inflammation-free state. Figure 2.3 shows the graphs of the variables 


\begin{tabular}{|l|l|}
\hline parameter & value \\
\hline \hline$P_{c}$ & 21.1 \\
$P_{i}$ & 2 \\
$P_{S}$ & 0.45 \\
$a_{p, 1}$ & 0.1752 \\
$a_{p, 2}$ & 0.0028 \\
$a_{i, 1}$ & 0.2336 \\
$a_{i, 2}$ & 0.0034 \\
$\kappa$ & 1.5 \\
$\alpha$ & 24.612 \\
$J_{U F}$ & 30 \\
$p_{\text {total }}$ & 24.6122 \\
$V_{p}^{0}$ & 4000 \\
$c_{p}^{0}$ & 73.4940 \\
$V_{i}^{0}$ & 17200 \\
$c_{i}^{0}$ & 24.5595 \\
\hline
\end{tabular}

Table 2.6: Parameter values and initial conditions from 13

\begin{tabular}{|c|c|}
\hline parameter & value \\
\hline \hline$L_{p}$ & 1.65 \\
$\sigma$ & 0.9 \\
\hline
\end{tabular}

Table 2.7: Values of $L_{p}$ and $\sigma$ from 13

\begin{tabular}{|c|c|}
\hline parameter & value \\
\hline \hline$a$ & -9.70 \\
$L$ & 22.35 \\
$d$ & 66.23 \\
$b$ & 0.9058 \\
$c$ & 0.0278 \\
\hline
\end{tabular}

Table 2.8: Nominal values of the parameters in the formulated functions for $L_{p}$ and $\sigma$

when $[C R P]=0.45 \times 10^{-3} \mathrm{mg} / \mathrm{mL}$. We see that the fluid and protein dynamics are very similar to the simulations in [13]. The one-hour treatment time is divided in three parts, each having 20 minutes. In the first and last 20 minutes of treatment, the ultrafiltration rate $J_{U F}=0$. For $t \in[40,60], J_{U F}=30 \mathrm{~mL} / \mathrm{min}$. We see in figure 2.3 that the states almost stay constant during the first 20 minutes, when $J_{U F}=0$. Then in the next 20 minutes, plasma and interstitial volumes decline. During this period, ultrafiltration is positive and fluid is taken out from the plasma compartment. Naturally, plasma volume declines. The volume of the interstitial compartment also goes down since vascular refilling is taking place; that is, fluid from the interstitial compartment shifts to the plasma compartment. Consequently, plasma and interstitial protein concentrations go up. In the final 20 minutes when $J_{U F}=0$ again, plasma volume goes back up while plasma protein concentration goes down. The interstitial volume continues its decline and the protein concentration continues its increase.

Using the same nominal parameter values, we simulate the progression of inflammation for HD patients over time by increasing the level of CRP. For each value of CRP concentration, we compute for the parameters $L_{p}$ and $\sigma$ using the functions 2.2 and (2.4). Table 2.9 shows that as CRP levels increase, $L_{p}$ increases and $\sigma$ decreases. Figure 2.4 shows the plots of the state variables for different CRP levels. 

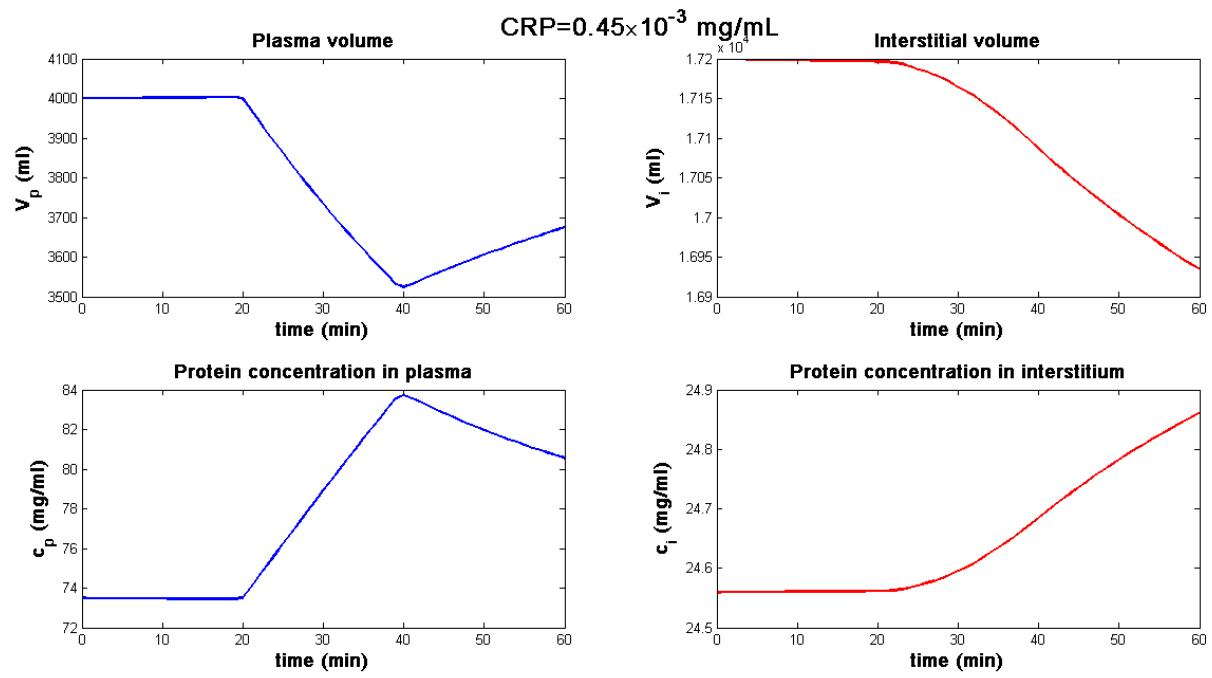

Figure 2.3: Plots of the state variable when $[C R P]=0.45 \times 10^{-3} \mathrm{mg} / \mathrm{mL}$

\begin{tabular}{|c|c|c|}
\hline $\mathrm{CRP}\left(\times 10^{-3}, \frac{m g}{m L}\right)$ & $L_{p}\left(\frac{m L}{m m H \cdot m i n}\right)$ & $\sigma$ \\
\hline \hline 0.45 & 1.6415 & 0.9056 \\
1 & 1.8449 & 0.9046 \\
3 & 2.5815 & 0.8953 \\
5 & 3.3086 & 0.8770 \\
8 & 4.3681 & 0.8338 \\
10 & 5.0460 & 0.7959 \\
12 & 5.6959 & 0.7518 \\
15 & 6.6103 & 0.6770 \\
20 & 7.9553 & 0.5398 \\
25 & 9.0665 & 0.4035 \\
\hline
\end{tabular}

Table 2.9: $L_{p}$ and $\sigma$ for different values of CRP

In general, when the fluid volume of a compartment decreases, then the protein concentration in that compartment tends to increase, and vice versa. In figure 2.4, we see four plots for each value of $\mathrm{CRP}$ concentration. The plots represent $V_{p}, c_{p}, V_{i}$, and $c_{i}$ over a period of 60 minutes. In the first 20 minutes when $J_{U F}=0$, we observe that $V_{p}$ goes down in an increasing rate as CRP levels increase. This is different from what is illustrated in figure 2.3, where $V_{p}$ remains approximately constant when $J_{U F}=0$. When ultrafiltration rate is turned on and set to $30 \mathrm{~mL} / \mathrm{min}, V_{p}$ decreases abruptly and reaches a minimum at around $t=40$. When ultrafiltration is turned off again in the last 20 minutes, $V_{p}$ gradually increases except when $[C R P]=0.025 \mathrm{mg} / \mathrm{mL}$, where it stays almost constant at a very low level.

The plasma protein concentration $c_{p}$ behaves in an opposite way as $V_{p}$. It increases 
during the first 40 minutes of the treatment, gradually in the first 20 minutes and then abruptly in the next 20 minutes, and then decreases when $J_{U F}=0$ in the remaining 20 minutes. For $t \in[20,40]$, when $J_{U F}$ is positive, there is an abrupt increase in $c_{p}$. This is expected since the plasma volume is declining fast during this period. As CRP concentration increases, the slope during the first 20 minutes becomes more steep and the maximum value that $c_{p}$ can reach also increases. This is true except for the case when $[C R P]=0.025 \mathrm{mg} / \mathrm{mL}$ where it appears that $c_{p}$ stays almost constant during the first 20 minutes, achieves a very sharp maximum at around 40 minutes, before it slightly decreases in the last 20 minutes.

The interstitial volume $V_{i}$ is increasing during the initial period of the treatment, reaching its peak at some time $t \in[20,30]$. Then it decreases for the remaining period. For low CRP concentration, the increase in $V_{i}$ is very small; it seems that $V_{i}$ stays almost constant before it declines. This can be observed for CRP concentration up to about $0.005 \mathrm{mg} / \mathrm{mL}$. For higher CRP concentrations, we see that $V_{i}$ increases during the initial period of treatment. As CRP concentration rises, the slopes become greater. This can be interpreted as: when there is a higher degree of inflammation based on CRP concentration, then there is greater leakage of fluid to the interstitial space which is visible to us through the increase in $V_{i}$ for $t \in[0,20]$. Another observation is that the interstitial volume $V_{i}$ at $t=60$ increases as CRP level increases. For instance, $V_{i}(60)=16915 \mathrm{~mL}$ when $[C R P]=0.001 \mathrm{mg} / \mathrm{mL}$, while $V_{i}(60)=17530 \mathrm{~mL}$ when $[C R P]=0.015 \mathrm{mg} / \mathrm{mL}$. Moreover, $V_{i}$ is higher at $t=60$ than at $t=0$, for $[C R P] \geq$ $0.015 \mathrm{mg} / \mathrm{mL}$. In other words, for $[C R P] \approx 0.015 \mathrm{mg} / \mathrm{mL}$ or greater, $V_{i}$ actually increased rather than decreased because its value after 60 minutes is higher than at $t=0$. However, we note that a HD session lasts for about four hours on the average and the simulations only represent a period of 60 minutes. Finally, we observe that when $[C R P]=0.025 \mathrm{mg} / \mathrm{mL}$, it seems that $V_{i}$ continues to slightly increase in the last 20 minutes of the treatment. This is not good since we aim to gradually reduce the amount of extra fluid in the extravascular compartment.

For CRP levels of up to $8 \mathrm{mg} / \mathrm{mL}, c_{i}$ behaves oppositely as $V_{i}$, where it decreases and reaches its minimum at some time $t \in[20,30]$ and then increases afterward. However, there is a change in the dynamics of $c_{i}$ during the first 20 minutes of treatment as CRP levels increase. From some CRP value around $0.010 \mathrm{mg} / \mathrm{mL}, c_{i}$ increases instead of decreases for the first 20 to 30 minutes. A possible explanation for this is that a larger $\mathrm{CRP}$ value, which is equivalent to a more severe inflammatory state, implies greater permeability. Consequently, there may also be a leakage of proteins from the plasma to the interstitial compartment which makes interstitial protein concentration rise. For high CRP concentration, that is, around $20 \mathrm{mg} / \mathrm{mL}$ or above, $c_{i}$ is increasing throughout the 60 -minute period until the slope slightly plateaus at $t=30$ onwards. 

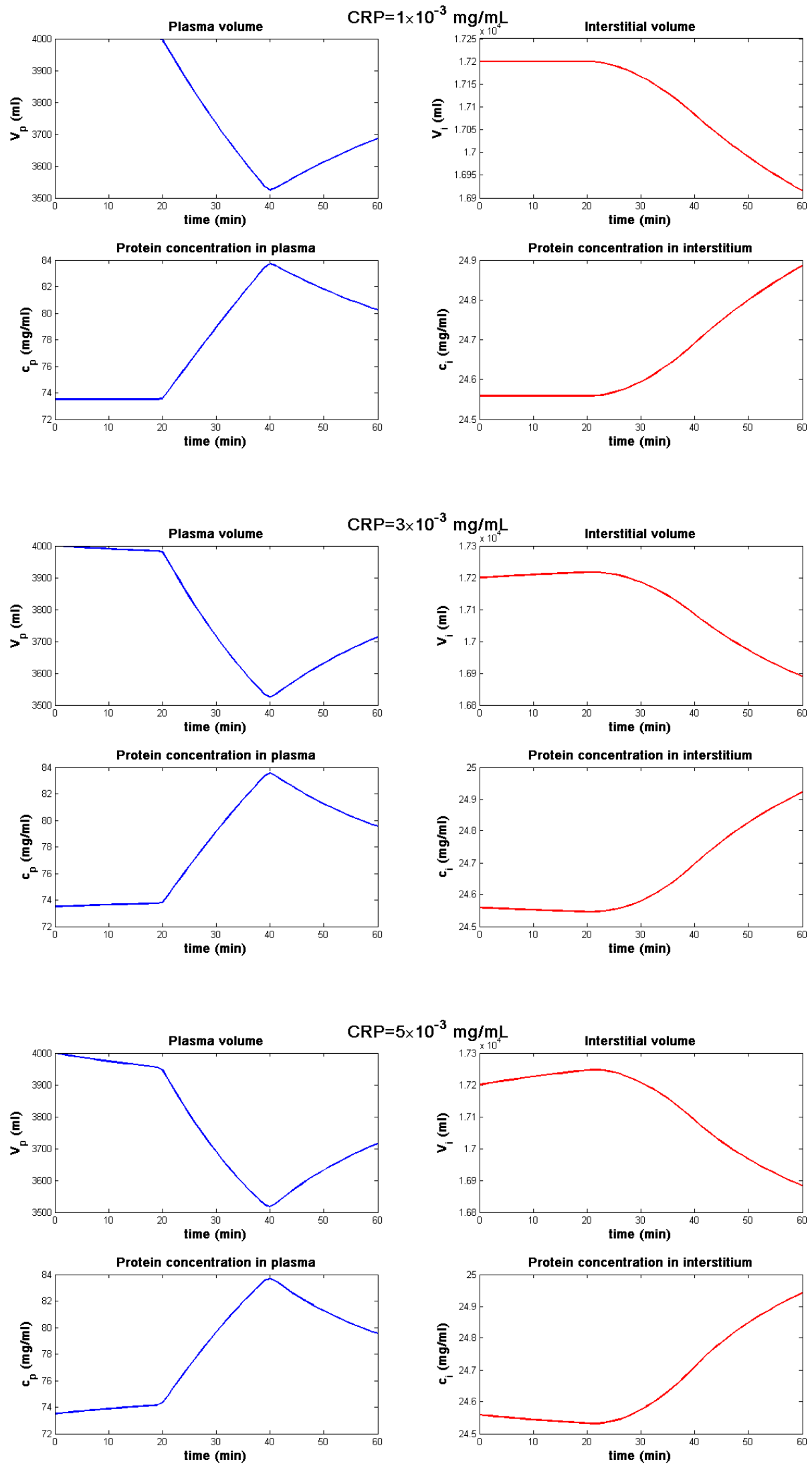

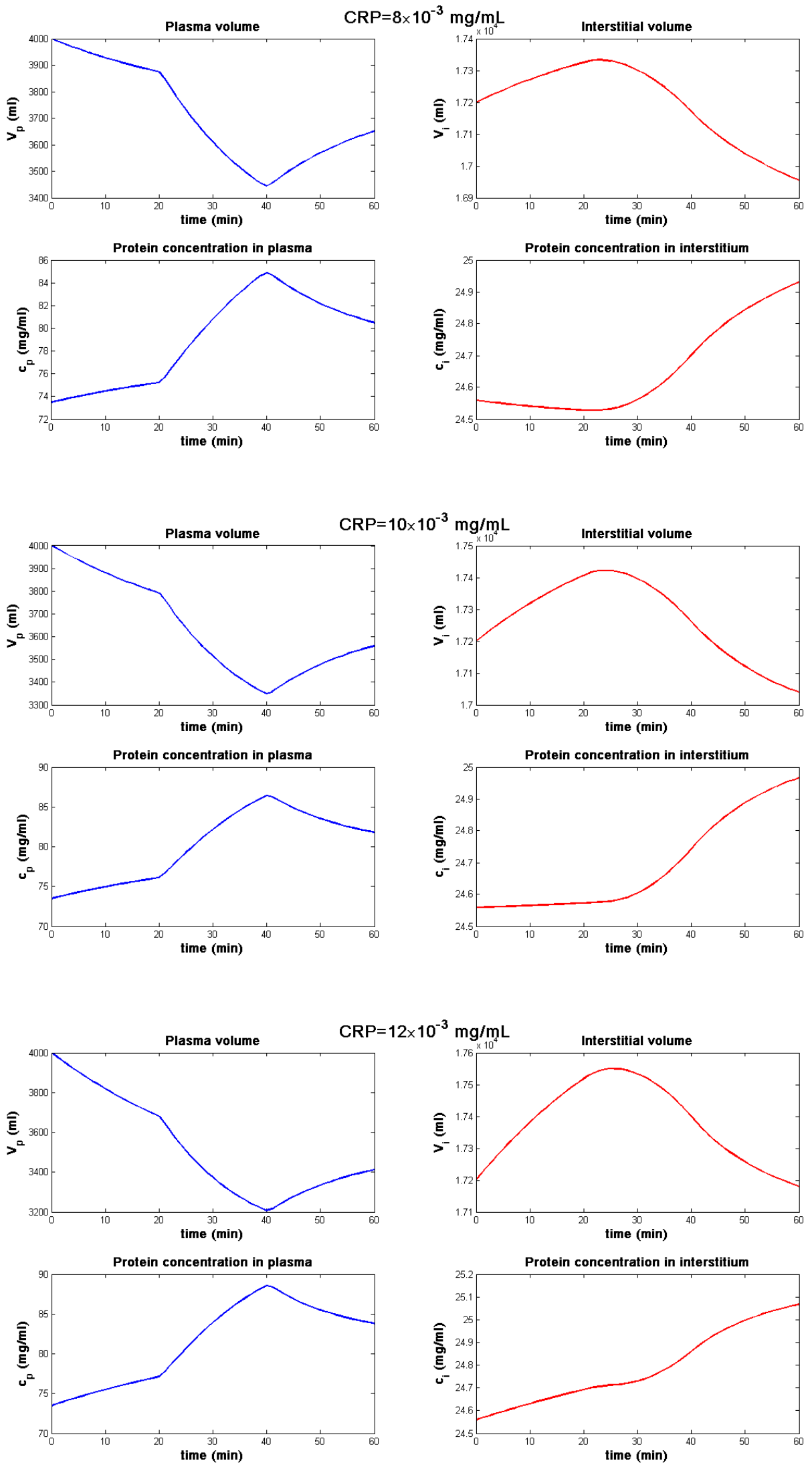

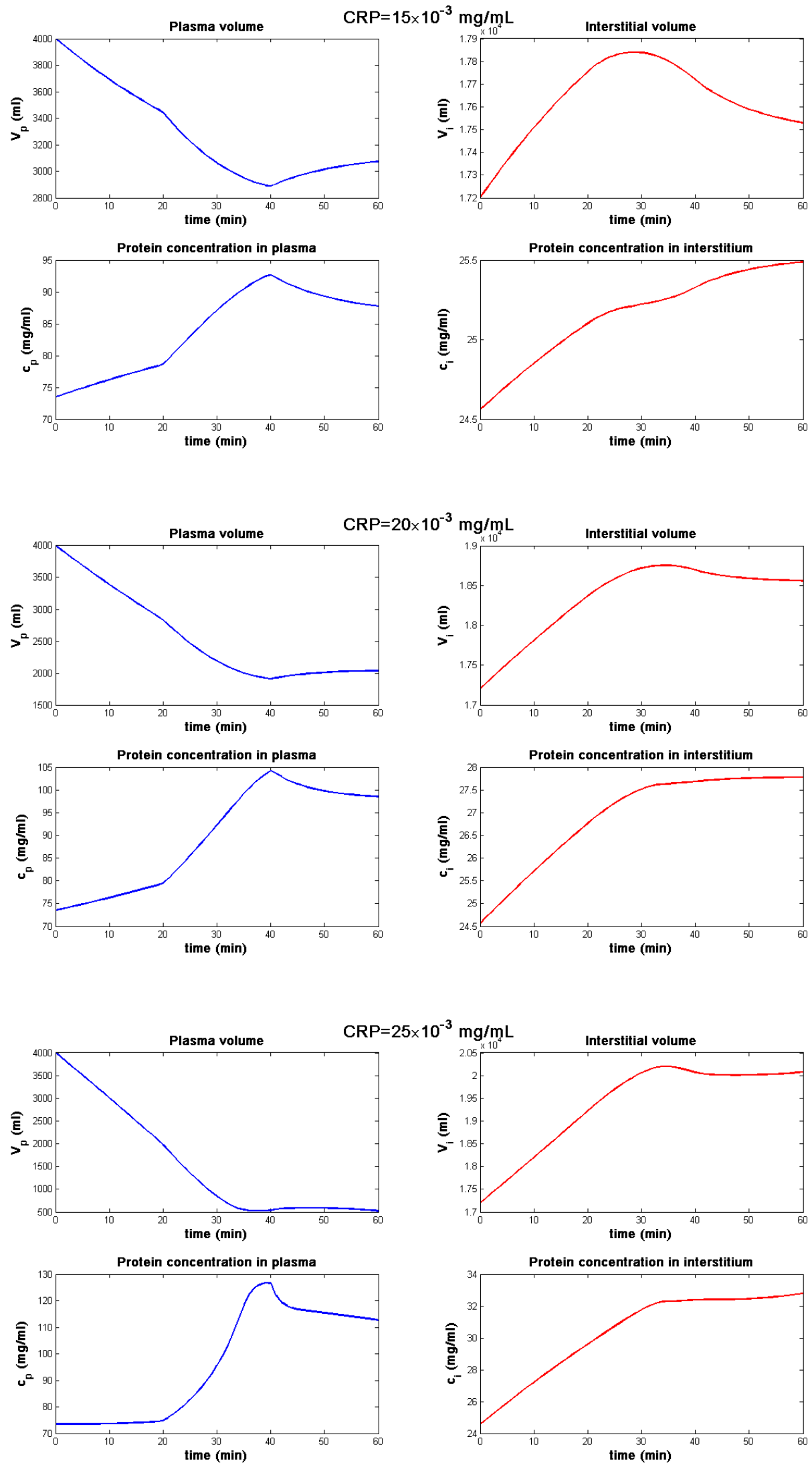

Figure 2.4: Plots of the state variables for increasing levels of CRP 
From the simulations, we observe that the fluid and protein concentration dynamics change as expected as we vary the level of CRP. But based on the behavior of $c_{i}$, there appears to be a leakage of proteins to the interstitial space for $[C R P] \approx 0.010 \mathrm{mg} / \mathrm{mL}$ and higher. Finally, it can also be said that the model may not any more be suitable for CRP values of about $0.025 \mathrm{mg} / \mathrm{mL}$ and greater. The plasma volume quickly drops to a very low value after the start of the treatment, plasma protein concentration had a sharp drop at some point, and interstitial volume is so much higher and continues to increase after one hour of treatment. A CRP level equal to $0.025 \mathrm{mg} / \mathrm{mL}$ is considered very high for patients on HD. 


\section{Chapter 3}

\section{Analysis and discussion}

In this chapter, we analyze the proposed model of vascular refilling with inflammation presented in the previous chapter. We apply sensitivity analysis, parameter estimation, and subset selection methods as discussed in the papers [86, 87, 88, 89]. Measurements for at least one output of the system are essential in our analyses. A model output function represents a measurable quantity of the system and is expressed in terms of the state variables and/or the parameters. In this study, we consider two model outputs: hematocrit (Hct) and fluid flux $\left(J_{v}\right)$. We focus our analysis on the five additional parameters $a, L, d, b$, and $c$, which were introduced to describe the relationships of CRP, $L_{p}$, and $\sigma$. This means that we fix the values of the other model parameters and assume that inflammation only influences the capillary wall. That is, the level of CRP only affects the five parameters associated with $L_{p}$ and $\sigma$. Estimates for $a, L, d, b$, and $c$ are calculated from generated measurements of hematocrit and fluid flux. Estimates from two outputs are done in the hope of obtaining improved parameter estimates. We use the values given in table 2.6 for the parameters we assumed to be fixed and the values in table 2.8 for the nominal values of $a, L, d, b$, and $c$.

During a hemodialysis treatment, hematocrit levels can be measured using a device called Crit-Line Monitor (CLM). Hematocrit is the percentage of blood consisting of cells [1. It is assumed that during HD, the mass of the red blood cells (RBC) in the vascular space is constant. As fluid is removed from the vascular space, hematocrit changes. The CLM also provides information on a patient's relative blood volume. On average, the hematocrit of men is around 42, while it is around 38 for women [1]. Most individuals with kidney disease have hematocrit levels which are less than the normal range. As a model output, hematocrit can be expressed in terms of the state variable $V_{p}$ in the following way

$$
H c t(t)=\frac{1}{H_{0} V_{p}(t)+1}
$$

where $H_{0}=\frac{1-H c t(0)}{H c t(0) V_{p}(0)}$, and $H c t(0)$ and $V_{p}(0)$ are the initial hematocrit and plasma 
volume, respectively.

Besides hematocrit, the fluid flux $J_{v}$ appearing in the proposed model is also considered as a model output. But unlike hematocrit, there is currently no device which provides measurements for fluid flux across the capillary membrane. But since we are interested in the parameters $a, L, d, b$, and $c$, and $J_{v}$ is directly related to $L_{p}$ and $\sigma$ where these parameters appear, then it is possible that more information about the model parameters may be obtained assuming that measurements for fluid flux are known. For this reason, we consider $J_{v}$ as another model output and see how the results compare with the results when hematocrit is used as the model output.

The following sections discuss theories on sensitivity analysis, subset selection, and parameter estimation using [86, 87, 88, 89] as main references. We then apply these methods on the proposed model with hematocrit and fluid flux as model outputs, and with respect to the parameters $a, L, d, b$ and $c$. Two cases are considered in the estimation of parameters: first, when CRP concentration is slightly elevated and second, when CRP concentration is high.

\subsection{Sensitivity analysis}

Traditional sensitivity functions (TSF), defined in terms of the derivatives of a model output with respect to the parameters, provide us information on how much a model output is influenced by changes in the parameter values. It gives an insight on which parameters have the most or the least influence to the dynamics of the model. Recognizing significant parameters on specific time intervals is useful for models involving a large number of parameters. This gives us an idea which among the parameters should be estimated more accurately, which parameters we expect to be more readily estimated from measurements, and on which time interval it is best to calculate the estimates from. A parameter having small sensitivities on some time interval $I$ means that the output does not carry much information about this parameter on $I$. Hence, it may be difficult to estimate this parameter using measurements taken on $I$. On the other hand, a parameter having large sensitivities on the interval $I$ means that the output has a lot of information about this parameter and we can expect that this parameter can be estimated more easily on $I$ than the other parameters. However, TSF do not indicate correlations between parameters. When parameters all have large sensitivities, it does not necessarily mean that all the parameters can be estimated with sufficient accuracy. It may happen that some of these parameters are related to each other or are redundant, and TSF are not enough to identify these occurrences. Sensitivities based on different model outputs may give different information about the influence of the same set of parameters to the model dynamics. This means that a parameter regarded as very influential based on its sensitivities using one model output may be regarded as 
less influential when considering another model output.

The following is a discussion of the traditional sensitivity functions. Later on, this will be applied to compute for the sensitivities of the model outputs hematocrit given in (3.1) and fluid flux $J_{v}$ given in (2.6) with respect to the parameters $a, L, d, b$, and $c$.

Let $x(\cdot) \in \mathbb{R}^{n}$ denote the vector of the state variables of the system, $\theta \in \mathcal{A} \subset \mathbb{R}^{p}$ be the vector of parameters belonging to an admissible set of parameter values $\mathcal{A}, \eta(\cdot) \in \mathbb{R}^{m}$ be the vector of outputs of the model, and $T$ be the length of time when measurements are taken. Consider the system and model output as follows:

$$
\begin{aligned}
\dot{x}(t) & =\mathcal{G}(t, x(t, \theta), \theta), \quad x(0)=x_{0}(\theta) \\
\eta(t) & =f(t, \theta), \quad 0 \leq t \leq T, \theta \in \mathcal{A},
\end{aligned}
$$

where $G \subset \mathbb{R}^{n}$ is open and $\mathcal{G}:[0, \infty) \times G \times \mathcal{A} \rightarrow \mathbb{R}^{n}, x_{0}: \mathcal{A} \rightarrow \mathbb{R}^{n}$, and $f:[0, T] \times \mathcal{A} \rightarrow \mathbb{R}^{m}$ are sufficiently smooth functions. Normally, the state variables also appear in the model output function and so, (3.3) can also be written as

$$
\eta(t)=h(t, x(t, \theta), \theta) \quad 0 \leq t \leq T, \theta \in \mathcal{A}
$$

where $h:[0, T] \times G \times \mathcal{A} \rightarrow \mathbb{R}^{m}$ is sufficiently smooth. If $x=x(t, \theta)$ is the solution to $(3.2)$, then

$$
f(t, \theta)=h(t, x(t, \theta), \theta) \quad 0 \leq t \leq T, \theta \in \mathcal{A} \text {. }
$$

In the following discussion, we assume a single model output; that is, $m=1$. Consider $0 \leq t \leq T$ and fix a nominal parameter vector $\theta_{0} \in \mathcal{A}$. In sensitivity analysis, it makes sense to use the relative or normalized sensitivity functions since we will be comparing sensitivities with respect to parameters possibly having different units. Denote by $s_{k}\left(t, \theta_{0}\right)$ the normalized sensitivity function of the model output function $f$ with respect to a parameter $\theta_{k}$ at $\theta=\theta_{0}$. Then $s_{k}\left(t, \theta_{0}\right)$ is given by

$$
\begin{aligned}
s_{k}\left(t, \theta_{0}\right) & =\frac{\theta_{0, k}}{f\left(t, \theta_{0}\right)} \frac{\partial f}{\partial \theta_{k}}\left(t, \theta_{0}\right), \\
& =\lim _{\Delta \theta_{k} \rightarrow 0} \frac{\left(f\left(t, \theta_{0}+\Delta \theta_{k} e_{k}\right)-f\left(t, \theta_{0}\right)\right) / f\left(t, \theta_{0}\right)}{\Delta \theta_{k} / \theta_{0, k}},
\end{aligned}
$$

where $\Delta \theta_{k}=\theta_{k}-\theta_{0, k}$ and $e_{k}$ is the $k$ th vector of the canonical basis of $\mathbb{R}^{p}, k=1, \ldots, p$. The factor $\frac{\theta_{0, k}}{f\left(t, \theta_{0}\right)}$ in 3.6 is necessary to make $s_{k}$ dimensionless, which is useful when doing a comparison among sensitivities with respect to different parameters.

To get $\frac{\partial f}{\partial \theta_{k}}(t, \theta)$ in $(3.6)$, we differentiate 3.5$)$ with respect to $\theta_{k}$. Using chain rule, we obtain

$$
\frac{\partial f}{\partial \theta_{k}}(t, \theta)=\frac{\partial h}{\partial x}(t, x(t, \theta), \theta) \frac{\partial x}{\partial \theta_{k}}(t, \theta)+\frac{\partial h}{\partial \theta_{k}}(t, x(t, \theta), \theta)
$$


Denote by $y(t):=\frac{\partial x}{\partial \theta_{k}}(t, \theta)$. Differentiating 3.2 with respect to $\theta_{k}$ and interchanging the order of derivatives, we get

$$
\begin{aligned}
\frac{\partial}{\partial t} \frac{\partial x}{\partial \theta_{k}}(t, \theta) & =\frac{\partial \mathcal{G}}{\partial x}(t, x(t, \theta), \theta) \frac{\partial x}{\partial \theta_{k}}(t, \theta)+\frac{\partial \mathcal{G}}{\partial \theta_{k}}(t, x(t, \theta), \theta) \\
\Leftrightarrow \dot{y}(t) & =\mathbb{A}(t, \theta) y(t)+g_{k}(t, \theta), \quad y(0)=\frac{\partial x_{0}}{\partial \theta_{k}}(\theta), \quad t \geq 0, \theta \in \mathcal{A}
\end{aligned}
$$

where

$$
\mathbb{A}(t, \theta)=\frac{\partial \mathcal{G}}{\partial x}(t, x(t, \theta), \theta) \text { and } g_{k}(t, \theta)=\frac{\partial \mathcal{G}}{\partial \theta_{k}}(t, x(t, \theta), \theta)
$$

Therefore, to get the sensitivities $s_{k}\left(t, \theta_{0}\right)$, one needs to solve first for $y$ from the following extended system:

$$
\begin{aligned}
\dot{x}(t) & =\mathcal{G}(t, x(t), \theta) \\
\dot{y}(t) & =\mathbb{A}(t, \theta) y(t)+g_{k}(t, \theta) \\
x(0) & =x_{0}(\theta), y(0)=\frac{\partial x_{0}}{\partial \theta_{k}}(\theta), \quad t \geq 0, \theta \in \mathcal{A} .
\end{aligned}
$$

When $y$ is determined, $\frac{\partial f}{\partial \theta_{k}}(t, \theta)$ can be computed from (3.7) and finally, $s_{k}\left(t, \theta_{0}\right)$ is obtained from (3.6).

In the next sections, we address the problem of parameter estimation and identifiability. The goal is to determine if the parameters $a, L, d, b$ and $c$ can be estimated with considerable accuracy from a given set of measurements on some time interval. The idea is to minimize the difference between the model output and the measurements in order to obtain the parameter estimates.

\subsection{Parameter estimation}

To simplify the notations, we again assume a single model output and so, $m=1$. Let $y_{j}$ be actual measurements of the system (3.2) and (3.3) taken at the sampling times $t_{j}, j=1, \ldots, N$ and $0 \leq t_{1}<\ldots<t_{N} \leq T$. Given measurements $y_{j}$, we want to find a parameter vector $\hat{\theta} \in \mathcal{A}$ such that the model outputs $f\left(t_{j}, \hat{\theta}\right)$ are as close to the measurements $y_{j}$ as possible, for $j=1, \ldots, N$. If the parameter vector $\hat{\theta}$ is unique, then we say that the parameters in $\hat{\theta}$ are identifiable.

Suppose that $y_{j}$ can be written in the form

$$
y_{j}=f\left(t_{j}, \theta_{0}\right)+\epsilon_{j}, \quad j=1, \ldots, N,
$$


where $\theta_{0}$ is the nominal parameter vector and $\epsilon_{j}$ is the measurement error. In equation (3.10), we assume that there exists a nominal or true parameter vector $\theta_{0}$ such that the measurements $y_{j}$ coincide with the model output $f$ at $t=t_{j}$ and $\theta=\theta_{0}$ plus some measurement error $\epsilon_{j}$, for $j=1, \ldots, N$. The measurement errors $\epsilon_{j}$ are assumed to be representations of a random variable $\mathcal{E}_{j}$, the measurement noise at $t_{j}$, for $j=1, \ldots, N$. It follows from 3.10 that $y_{j}$ are also representations of a random variable $\mathcal{Y}_{j}$, the measurement process at $t_{j}$ and we write

$$
\mathcal{Y}_{j}=f\left(t_{j}, \theta_{0}\right)+\mathcal{E}_{j}, \quad j=1, \ldots, N
$$

Equation (3.11) is referred to as the statistical model corresponding to the model output (3.3). Furthermore, we assume that the random variables $\mathcal{E}_{j}, j=1, \ldots, N$ satisfy the following conditions:

a) $\mathcal{E}_{j}$ are independent, that is, $\operatorname{Cov}\left(\mathcal{E}_{j}, \mathcal{E}_{i}\right)=0$ when $j \neq i$, and identically distributed (i.i.d.);

b) the expected value of $\mathcal{E}_{j}$ is zero: $\mathbb{E}\left(\mathcal{E}_{j}\right)=0$;

c) and the variance of $\mathcal{E}_{j}$ is common and finite: $\operatorname{Var}\left(\mathcal{E}_{j}\right)=\sigma_{0}^{2}<\infty$.

From these assumptions and from (3.11), it follows that for $j=1, \ldots, N, \mathbb{E}\left(\mathcal{Y}_{j}\right)=$ $f\left(t_{j}, \theta_{0}\right)$ and $\operatorname{Var}\left(\mathcal{Y}_{j}\right)=\sigma_{0}^{2}$.

Let

$$
\begin{aligned}
F(\theta) & =\left[f\left(t_{1}, \theta\right), \ldots, f\left(t_{N}, \theta\right)\right]^{T}, \\
\epsilon & =\left[\epsilon_{1}, \ldots, \epsilon_{N}\right]^{T}, \\
\text { and } y & =\left[y_{1}, y_{2}, \ldots, y_{N}\right]^{T}=F\left(\theta_{0}\right)+\epsilon .
\end{aligned}
$$

To determine the parameter vector $\hat{\theta}$ such that the errors $\epsilon_{j}$ are as close to zero as possible, we consider a weighted least squares cost functional given by

$$
\begin{aligned}
J(\theta) & =\frac{1}{2 \sigma_{0}^{2}} \sum_{j=1}^{N}\left(y_{j}-f\left(t_{j}, \theta\right)\right)^{T}\left(y_{j}-f\left(t_{j}, \theta\right)\right) \\
& =\frac{1}{2 \sigma_{0}^{2}}\|y-F(\theta)\|^{2} .
\end{aligned}
$$

The functional $J$ represents the residuals between the measurements and the model output values. We are therefore interested in a parameter $\hat{\theta}$ that gives the minimum value of $J$ in 3.12 . That is, we want to determine

$$
\hat{\theta}=\underset{\theta \in \mathcal{A}}{\operatorname{argmin}} J(\theta) .
$$

Note that $\hat{\theta}$ is also a representation of a random variable $\hat{\Theta}$ since the parameter estimates $\hat{\theta}$ vary as the measurement errors. The random variable $\hat{\Theta}$ is called the weighted least 
squares estimator for the parameter estimation problem. Thus, the estimates depend on the statistical properties of this random variable $\hat{\Theta}$.

We first discuss briefly the first and second-order conditions for the minimizer $\hat{\theta}$. If $\hat{\theta}$ is a minimizer of $(3.12)$, then it must satisfy the first-order condition

$$
\left.\operatorname{grad}_{\theta} J(\theta)\right|_{\theta=\hat{\theta}}=0
$$

From 3.12 , we get

$$
\begin{array}{r}
\frac{\partial J}{\partial \theta_{k}}(\theta)=-\frac{1}{\sigma_{0}^{2}}(y-F(\theta))^{T}\left(\frac{\partial F}{\partial \theta_{k}}\right)(\theta), \quad \text { for } k=1, \ldots, N \\
\Rightarrow \operatorname{grad}_{\theta} J(\theta)=-\frac{1}{\sigma_{0}^{2}}(y-F(\theta))^{T}\left(\frac{\partial F}{\partial \theta}\right)(\theta), \quad \theta \in \mathcal{A} .
\end{array}
$$

Equivalently, condition (3.14) can be expressed as

$$
(y-F(\hat{\theta}))^{T}\left(\frac{\partial F}{\partial \theta}\right)(\hat{\theta})=0 .
$$

In addition to (3.14), the second-order necessary condition for $\hat{\theta}$ to be a minimizer of $J$ is that the Hessian matrix $H$ at $\theta=\hat{\theta}$ is positive semi-definite. That is, $H(\hat{\theta}) \geq 0$, where $H$ is given by

$$
\begin{aligned}
H(\theta) & =\left(\left(\frac{\partial^{2} J}{\partial \theta_{j} \partial \theta_{k}}\right)(\theta)\right)_{j, k=1, \ldots, p}, \quad \theta \in \mathcal{A} \\
& =\frac{1}{\sigma_{0}^{2}}\left(\frac{\partial F}{\partial \theta}(\theta)\right)^{T}\left(\frac{\partial F}{\partial \theta}(\theta)\right)-\frac{1}{\sigma_{0}^{2}}\left((y-F(\theta))^{T} \frac{\partial^{2} F}{\partial \theta_{j} \partial \theta_{k}}(\theta)\right)_{j, k=1, \ldots, p} .
\end{aligned}
$$

If the differences $y_{j}-f\left(t_{j}, \theta\right)$ for $j=1, \ldots, N$ are small, then $H$ can be approximated by the first term in (3.15). That is,

$$
H(\theta) \approx \frac{1}{\sigma_{0}^{2}}\left(\frac{\partial F}{\partial \theta}(\theta)\right)^{T}\left(\frac{\partial F}{\partial \theta}(\theta)\right)=: \mathcal{F}(\theta) .
$$

This approximation holds for small $\epsilon_{j}, j=1, \ldots, N$ and $\theta$ close to $\theta_{0}$. The matrix $\mathcal{F}(\theta)$ is called the Fisher information matrix (FIM). The FIM $\mathcal{F}(\theta)$ is a $p \times p$ symmetric, positive semi-definite matrix for any $\theta \in \mathcal{A}$ and has an important role in parameter estimation and identification.

Next, define the $N \times p$ sensitivity matrix $\chi(\theta)$ by

$$
\chi(\theta)=\left(\frac{\partial f\left(t_{j}\right)}{\partial \theta_{i}}\right)_{i=1, \ldots, p, j=1, \ldots, N}=\left(\begin{array}{c}
\nabla_{\theta} f\left(t_{1}, \theta\right) \\
\vdots \\
\nabla_{\theta} f\left(t_{N}, \theta\right)
\end{array}\right),
$$


where $\nabla_{\theta} f\left(t_{j}, \theta\right), j=1, \ldots, N$ is a $p$-dimensional row vector. The FIM defined in (3.16) can also be written in terms of the sensitivity matrix

$$
\mathcal{F}(\theta)=\frac{1}{\sigma_{0}^{2}} \chi(\theta)^{T} \chi(\theta)
$$

It was shown in 87 that using asymptotic theory, that is, as $N \rightarrow \infty$, and assuming that the estimate $\hat{\theta}$ is close to $\theta_{0}$ for sufficiently large $N$,

$$
\begin{aligned}
\mathbb{E}(\hat{\Theta}) & \approx \theta_{0}, \\
\operatorname{Cov}(\hat{\Theta}) & \approx \sigma_{0}^{2}\left(\chi\left(\theta_{0}\right)^{T} \chi\left(\theta_{0}\right)\right)^{-1}=\mathcal{F}\left(\theta_{0}\right)^{-1}=: \Sigma_{0}, \\
\text { and } S E_{k} & =\sqrt{\left(\Sigma_{0}\right)_{k k}}, \quad k=1, \ldots, p,
\end{aligned}
$$

where the standard errors $S E_{k}$ are given by the square root of the diagonal entries of the covariance matrix $\Sigma_{0}$. The relation (3.17) means that the estimator $\hat{\Theta}$ is unbiased. The unknown error variance $\sigma_{0}^{2}$ was also shown to be approximately given by

$$
\sigma_{0}^{2} \approx \frac{1}{N-p} \sum_{j=1}^{N}\left(y_{j}-f\left(t_{j}, \theta_{0}\right)\right)^{2}=\frac{1}{N-p}\left\|y-F\left(\theta_{0}\right)\right\|_{2}^{2} .
$$

Using standard errors is one approach to quantify the quality of the estimates. This will be the basis for selecting the best parameter subsets among a set of parameters, which is discussed in the next section.

\subsection{Subset selection algorithm}

Especially for models having many parameters, it is difficult to obtain reliable estimates of all the parameters at the same time. To improve parameter estimation and identification, it may be helpful to determine estimates on a subset of the whole set of parameters. A subset selection algorithm described in [86, 88] is presented in this section. The algorithm utilizes the sensitivity matrix and asymptotic standard errors in choosing the best parameter subset of a desired length. From $p_{0}$ parameters, we select parameter vector subsets containing $p$ parameters, $p<p_{0}$, such that the sensitivity matrix corresponding to the $p$ parameters has full rank. Then we compute for the asymptotic standard error and use this as a measure of how well a parameter subset can be estimated.

Below is the algorithm used in this study. Here, $p_{0}=5, p \in\{2,3,4\}$, and the vector $\left[\theta_{1}, \theta_{2}, \theta_{3}, \theta_{4}, \theta_{5}\right]=[a, L, d, b, c]$. Nominal parameter values and nominal variance are needed to implement the algorithm. For a given $p$, we do the following steps:

1. Combinatorial search. We construct the set $\mathcal{S}_{p}$ containing all the possible parameter vectors $\theta \in \mathbb{R}^{p}$, such that the components $\theta_{j_{i}}$ of each vector $\theta$ have indices from $\{1,2,3,4,5\}$;

$$
\mathcal{S}_{p}=\left\{\theta=\left[\theta_{j_{1}}, \ldots, \theta_{j_{p}}\right] \in \mathbb{R}^{p}: j_{i} \in\{1,2,3,4,5\}, j_{i} \neq j_{k} \forall i, k=1, \ldots, p\right\} .
$$


2. Full rank test. Construct the set of viable parameters $\Theta_{p} \subseteq \mathcal{S}_{p}$ by choosing the parameter subsets from $\mathcal{S}_{p}$ having sensitivity matrices with full rank. That is,

$$
\Theta_{p}=\left\{\theta: \theta \in \mathcal{S}_{p}, \operatorname{rank}(\chi(\theta))=p\right\} .
$$

3. Standard error test. For each $\theta \in \Theta_{p}$, determine the covariance matrix $\Sigma(\theta)$

$$
\Sigma(\theta)=\sigma_{0}^{2}\left(\chi(\theta)^{T} \chi(\theta)\right)^{-1}=(\mathcal{F}(\theta))^{-1} \in \mathbb{R}^{p \times p} .
$$

Then calculate the vector of coefficients of variation for $\theta$, denoted by $v(\theta) \in \mathbb{R}^{p}$, with components given by

$$
v_{i}(\theta)=\frac{\sqrt{\Sigma(\theta)_{i i}}}{\left(\theta_{0}\right)_{i}} \text { for } i=1, \ldots, p .
$$

The $i t h$ component of $v$ is equal to the ratio of the standard error of the $i$ th parameter to its corresponding nominal parameter value. Finally, calculate the parameter selection score $\alpha(\theta)$ by

$$
\alpha(\theta)=\|v(\theta)\|
$$

A small parameter selection score $\alpha$ means low uncertainty possibilities of the estimates, while a large value of $\alpha$ means that there is a large uncertainty for at least one of the estimates. Hence, in choosing the best parameter subsets of length $p$, we prefer those having small selection scores.

The condition number $\kappa$ of a full rank sensitivity matrix $\chi(\theta)$ is the ratio of the largest to the smallest singular value of $\chi(\theta)$. A low condition number indicates that the matrix is well-conditioned, while a high condition number means that the matrix is ill-conditioned. If $\kappa(\chi(\theta))$ is low, then the condition number of the Fisher information matrix $\mathcal{F}(\theta)=\frac{1}{\sigma_{0}^{2}} \chi^{T}(\theta) \chi(\theta)$ is also low. When $\kappa(\chi(\theta))$ and $\alpha(\theta)$ are relatively small, then the parameter vector $\theta$ is said to be feasible. A feasible parameter vector can be estimated from data with a small uncertainty [86].

It is noteworthy to mention that besides using standard errors as the basis for choosing the best parameter subset, one can also use other criteria utilizing the Fisher information matrix. The D-,C-, and E-optimal designs make use of the determinant, inverse, and eigenvalue of $\mathcal{F}(\theta)$, respectively, in order to obtain the best and accurate estimates from available measurements 86,87 .

\subsection{Traditional sensitivity functions of the model}

In this section, we compute for the sensitivities of the model outputs hematocrit $H c t$ and fluid flux $J_{v}$ with respect to the parameters $a, L, d, b$, and $c$. This provides us information 
on which parameters have the most or the least influence on the model dynamics during the first hour of HD. We also observe how the sensitivities change as the level of CRP concentration increases. The nominal values used in the numerical simulations are given in tables 2.6 and 2.8 .

\subsubsection{Sensitivities with respect to hematocrit}

Let $x(\cdot) \in \mathbb{R}^{4}$ be the vector of states, $\theta \in \mathcal{A} \subset \mathbb{R}^{5}$ be the vector of parameters, and $h(\cdot) \in \mathbb{R}$ be the output of the model, which in this case is the hematocrit. That is, we have

$$
\begin{aligned}
& x(t, \theta)=\left[\begin{array}{llll}
V_{p} & c_{p} & V_{i} & c_{i}
\end{array}\right]^{T} \\
& \theta=\left[\begin{array}{lllll}
\theta_{1} & \theta_{2} & \theta_{3} & \theta_{4} & \theta_{5}
\end{array}\right]^{T}=\left[\begin{array}{lllll}
a & L & d & b & c
\end{array}\right]^{T} \\
& h(t, x(t, \theta))=\frac{1}{H_{0} V_{p}(t, \theta)+1} \text {, where } H_{0}=\frac{1-H c t(0)}{\operatorname{Hct}(0) V_{p}(0)} \text {, }
\end{aligned}
$$

for $0 \leq t \leq T=60$ and $\theta \in \mathcal{A}$.

From the output function (3.18), we get the following

$$
\begin{aligned}
& \operatorname{grad}_{x} h(t, x)=\left[\begin{array}{llll}
-\frac{H_{0}}{\left(H_{0} V_{p}(t, \theta)+1\right)^{2}} & 0 & 0 & 0
\end{array}\right] \\
& \frac{\partial h}{\partial \theta_{k}}(t, x)=0 \quad \text { for } k=1,2,3,4,5 .
\end{aligned}
$$

It follows that for $k=1,2,3,4,5,3.7$ becomes

$$
\frac{\partial f}{\partial \theta_{k}}(t, \theta)=-\frac{H_{0}}{\left(H_{0} V_{p}(t, \theta)+1\right)^{2}} \frac{\partial V_{p}}{\partial \theta_{k}}
$$

and $(3.6)$ simplifies to

$$
s_{k}\left(t, \theta_{0}\right)=-\frac{\theta_{0, k} H_{0}}{H_{0} V_{p}\left(t, \theta_{0}\right)+1} \frac{\partial V_{p}}{\partial \theta_{k}}\left(t, \theta_{0}\right) .
$$

Next, we compute for the matrices $\mathbb{A}$ and $g_{k}$ appearing in (3.8). We drop the parentheses from the functions to simplify the notations. From the proposed model (2.5), $\mathcal{G}$ is given by

$$
\mathcal{G}(x, t, \theta)=\left[\begin{array}{c}
J_{v}+\kappa-J_{U F} \\
\frac{J_{s}-c_{p}\left(J_{v}+\kappa-J_{U F}\right)}{V_{p}} \\
-J_{v}-\kappa \\
\frac{-J_{s}+c_{i}\left(J_{v}+\kappa\right)}{V_{i}}
\end{array}\right]
$$


Denote the following expressions by

$$
\begin{aligned}
J_{s}^{+} & :=c_{i}(t)-\frac{c_{p}-c_{i}}{e^{x}-1} \\
J_{s}^{-} & :=c_{p}(t)-\frac{c_{p}-c_{i}}{e^{x}-1} \\
A & :=\frac{\left(e^{x}-1\right)-\left(c_{p}-c_{i}\right) e^{x} g_{x}^{12}(1-\sigma) P_{S}^{-1}}{\left(e^{x}-1\right)^{2}} \\
B & :=\frac{-\left(e^{x}-1\right)-\left(c_{p}-c_{i}\right) e^{x} g_{x}^{14}(1-\sigma) P_{S}^{-1}}{\left(e^{x}-1\right)^{2}} .
\end{aligned}
$$

Then $\mathbb{A}(t, \theta)=\frac{\partial \mathcal{G}}{\partial x}(t, x(t, \theta), \theta)$ is given by the matrix

$$
\mathcal{G}_{x}(x, t, \theta)=\left[\begin{array}{cccc}
0 & g_{x}^{12} & 0 & g_{x}^{14} \\
g_{x}^{21} & g_{x}^{22} & 0 & g_{x}^{24} \\
0 & g_{x}^{32} & 0 & g_{x}^{34} \\
0 & g_{x}^{42} & g_{x}^{43} & g_{x}^{44}
\end{array}\right]
$$

where

$$
\begin{aligned}
& g_{x}^{21}=-\frac{1}{V_{p}^{2}}\left(J_{s}-c_{p}\left(J_{v}+\kappa-J_{U F}\right)\right) \\
& g_{x}^{12}= \frac{\partial J_{v}}{\partial c_{p}}=L_{p} \sigma\left(a_{p, 1}+2 a_{p, 2} c_{p}\right) \\
& g_{x}^{22}= \begin{cases}\frac{1}{V_{p}}\left[g_{x}^{12}(1-\sigma) J_{s}^{+}-J_{v}(1-\sigma) A-\left(J_{v}+\kappa-J_{U F}\right)-c_{p} g_{x}^{12}\right], & J_{v}>0 \\
\frac{1}{V_{p}}\left[-P_{S}-\left(J_{v}+\kappa-J_{U F}\right)-c_{p} g_{x}^{12}\right], & J_{v}=0 \\
\frac{1}{V_{p}}\left[g_{x}^{12}(1-\sigma) J_{s}^{-}+J_{v}(1-\sigma)(1-A)-\left(J_{v}+\kappa-J_{U F}\right)-c_{p} g_{x}^{12}\right], & J_{v}<0\end{cases} \\
& g_{x}^{32}=-g_{x}^{12} \\
& g_{x}^{42}= \begin{cases}\frac{1}{V_{i}}\left[-g_{x}^{12}(1-\sigma) J_{s}^{+}+J_{v}(1-\sigma) A+c_{i} g_{x}^{12}\right], & J_{v}>0 \\
\frac{1}{V_{i}}\left[P_{S}+c_{i} g_{x}^{12}\right], & \\
\frac{1}{V_{i}}\left[-g_{x}^{12}(1-\sigma) J_{s}^{-}-J_{v}(1-\sigma)(1-A)+c_{i} g_{x}^{12}\right], & J_{v}<0\end{cases} \\
& g_{x}^{43}=-\frac{1}{V_{i}^{2}}\left(-J_{s}+c_{i}\left(J_{v}+\kappa\right)\right)
\end{aligned}
$$




$$
\begin{aligned}
& g_{x}^{14}= \frac{\partial J_{v}}{\partial c_{i}}=-L_{p} \sigma\left(a_{i, 1}+2 a_{i, 2} c_{i}\right) \\
& g_{x}^{24}= \begin{cases}\frac{1}{V_{p}}\left[g_{x}^{14}(1-\sigma) J_{s}^{+}+J_{v}(1-\sigma)(1-B)-c_{p} g_{x}^{14}\right], & J_{v}>0 \\
\frac{1}{V_{p}}\left[P_{S}-c_{p} g_{x}^{14}\right], & J_{v}=0 \\
\frac{1}{V_{p}}\left[g_{x}^{14}(1-\sigma) J_{s}^{-}-J_{v}(1-\sigma) B-c_{p} g_{x}^{14}\right], & J_{v}<0\end{cases} \\
& g_{x}^{34}=-g_{x}^{14} \\
& g_{x}^{44}= \begin{cases}\frac{1}{V_{i}}\left[-g_{x}^{14}(1-\sigma) J_{s}^{+}-J_{v}(1-\sigma)(1-B)+\left(J_{v}+\kappa\right)+c_{i} g_{x}^{14}\right], & J_{v}>0 \\
\frac{1}{V_{i}}\left[-P_{S}+\left(J_{v}+\kappa\right)+c_{i} g_{x}^{14}\right], & J_{v}=0 \\
\frac{1}{V_{i}}\left[-g_{x}^{14}(1-\sigma) J_{s}^{-}+J_{v}(1-\sigma) B+\left(J_{v}+\kappa\right)+c_{i} g_{x}^{14}\right], & J_{v}<0\end{cases}
\end{aligned}
$$

For $k=1,2,3,4,5$,

$$
g_{k}(t, \theta)=\frac{\partial \mathcal{G}}{\partial \theta_{k}}(t, x(t, \theta), \theta)=\left[\begin{array}{c}
g_{\theta}^{1 k} \\
g_{\theta}^{2 k} \\
g_{\theta}^{3 k} \\
g_{\theta}^{4 k}
\end{array}\right]
$$

where

$$
\begin{aligned}
& g_{\theta}^{11}=\frac{\partial J_{v}}{\partial a}=\sigma\left(\pi_{p}-\pi_{i}\right)-\left(P_{c}-P_{i}\right) \\
& g_{\theta}^{12}=\frac{\partial J_{v}}{\partial L}=\frac{\sigma\left(\pi_{p}-\pi_{i}\right)-\left(P_{c}-P_{i}\right)}{1+e^{-d[C R P]}} \\
& g_{\theta}^{13}=\frac{\partial J_{v}}{\partial d}=\left(\sigma\left(\pi_{p}-\pi_{i}\right)-\left(P_{c}-P_{i}\right)\right) \frac{L[C R P] e^{-d[C R P]}}{\left(1+e^{-d[C R P]}\right)^{2}} \\
& g_{\theta}^{14}=\frac{\partial J_{v}}{\partial b}=L_{p} e^{-\left(\frac{[C R P]}{c}\right)^{2}}\left(\pi_{p}-\pi_{i}\right) \\
& g_{\theta}^{15}=\frac{\partial J_{v}}{\partial c}=L_{p} D\left(\pi_{p}-\pi_{i}\right)
\end{aligned}
$$

and denote by

$$
\begin{aligned}
C & :=\frac{\partial J_{s}^{+}}{\partial b}=\frac{\partial J_{s}^{-}}{\partial b}=\frac{\left(c_{p}-c_{i}\right) e^{x}\left(g_{\theta}^{14}(1-\sigma)-J_{v} e^{\left.-\left(\frac{[C R P]}{c}\right)^{2}\right)}\right.}{P_{S}\left(e^{x}-1\right)^{2}} \\
D & :=\frac{\partial \sigma}{\partial c}=2 b \frac{[C R P]^{2}}{c^{3}} e^{-\left(\frac{[C R P]}{c}\right)^{2}} \\
E & :=\frac{\partial J_{s}^{+}}{\partial c}=\frac{\partial J_{s}^{-}}{\partial c}=\frac{\left(c_{p}-c_{i}\right) e^{x}\left(g_{\theta}^{15}(1-\sigma)-J_{v} D\right)}{P_{S}\left(e^{x}-1\right)^{2}}
\end{aligned}
$$


For $j=1,2,3$, we have

$$
\begin{aligned}
& g_{\theta}^{2 j}= \begin{cases}\frac{1}{V_{p}}\left[g_{\theta}^{1 j}(1-\sigma) J_{s}^{+}+J_{v}(1-\sigma) \frac{\left(c_{p}-c_{i}\right) e^{x} g_{\theta}^{1 j}(1-\sigma)}{P_{S}\left(e^{x}-1\right)^{2}}-c_{p} g_{\theta}^{1 j}\right], & J_{v}>0 \\
\frac{1}{V_{p}}\left[-c_{p} g_{\theta}^{1 j}\right], & J_{v}=0 \\
\frac{1}{V_{p}}\left[g_{\theta}^{1 j}(1-\sigma) J_{s}^{-}+J_{v}(1-\sigma) \frac{\left(c_{p}-c_{i}\right) e^{x} g_{\theta}^{1 j}(1-\sigma)}{P_{S}\left(e^{x}-1\right)^{2}}-c_{p} g_{\theta}^{1 j}\right], & J_{v}<0\end{cases} \\
& g_{\theta}^{3 j}=-g_{\theta}^{1 j} \\
& g_{\theta}^{4 j}= \begin{cases}\frac{1}{V_{i}}\left[-g_{\theta}^{1 j}(1-\sigma) J_{s}^{+}-J_{v}(1-\sigma) \frac{\left(c_{p}-c_{i}\right) e^{x} g_{\theta}^{1 j}(1-\sigma)}{P_{S}\left(e^{x}-1\right)^{2}}+c_{i} g_{\theta}^{1 j}\right], & J_{v}>0 \\
\frac{1}{V_{i}}\left[c_{i} g_{\theta}^{1 j}\right], & J_{v}=0 . \\
\frac{1}{V_{i}}\left[-g_{\theta}^{1 j}(1-\sigma) J_{s}^{-}-J_{v}(1-\sigma) \frac{\left(c_{p}-c_{i}\right) e^{x} g_{\theta}^{1 j}(1-\sigma)}{P_{S}\left(e^{x}-1\right)^{2}}+c_{i} g_{\theta}^{1 j}\right], & J_{v}<0\end{cases}
\end{aligned}
$$

The rest of the terms are given by

$$
\begin{array}{ll}
g_{\theta}^{24}= \begin{cases}\frac{1}{V_{p}}\left[g_{\theta}^{14}(1-\sigma) J_{s}^{+}-J_{v} e^{-\left(\frac{[C R P]}{c}\right)^{2}} J_{s}^{+}+J_{v}(1-\sigma) C-c_{p} g_{\theta}^{14}\right], & J_{v}>0 \\
\frac{1}{V_{p}}\left[-c_{p} g_{\theta}^{14}\right], & J_{v}=0 \\
\frac{1}{V_{p}}\left[g_{\theta}^{14}(1-\sigma) J_{s}^{-}-J_{v} e^{-\left(\frac{[C R P]}{c}\right)^{2}} J_{s}^{-}+J_{v}(1-\sigma) C-c_{p} g_{\theta}^{14}\right], & J_{v}<0\end{cases} \\
g_{\theta}^{34}=-g_{\theta}^{14} &
\end{array}
$$$$
g_{\theta}^{44}= \begin{cases}\frac{1}{V_{i}}\left[-g_{\theta}^{14}(1-\sigma) J_{s}^{+}+J_{v} e^{-\left(\frac{[C R P]}{c}\right)^{2}} J_{s}^{+}-J_{v}(1-\sigma) C+c_{i} g_{\theta}^{14}\right], & J_{v}>0 \\ \frac{1}{V_{i}}\left[c_{i} g_{\theta}^{14}\right], & J_{v}=0 \\ \frac{1}{V_{i}}\left[-g_{\theta}^{14}(1-\sigma) J_{s}^{-}+J_{v} e^{-\left(\frac{[C R P]}{c}\right)^{2}} J_{s}^{-}-J_{v}(1-\sigma) C+c_{i} g_{\theta}^{14}\right], & J_{v}<0\end{cases}
$$$$
g_{\theta}^{25}= \begin{cases}\frac{1}{V_{p}}\left[g_{\theta}^{15}(1-\sigma) J_{s}^{+}-J_{v} D J_{s}^{+}+J_{v}(1-\sigma) E-c_{p} g_{\theta}^{15}\right], & J_{v}>0 \\ \frac{1}{V_{p}}\left[-c_{p} g_{\theta}^{15}\right], & J_{v}=0 \\ \frac{1}{V_{p}}\left[g_{\theta}^{15}(1-\sigma) J_{s}^{-}-J_{v} D J_{s}^{-}+J_{v}(1-\sigma) E-c_{p} g_{\theta}^{15}\right], & J_{v}<0\end{cases}
$$$$
g_{\theta}^{35}=-g_{\theta}^{15}
$$$$
g_{\theta}^{45}= \begin{cases}\frac{1}{V_{i}}\left[-g_{\theta}^{15}(1-\sigma) J_{s}^{+}+J_{v} D J_{s}^{+}-J_{v}(1-\sigma) E+c_{i} g_{\theta}^{15}\right], & J_{v}>0 \\ \frac{1}{V_{i}}\left[c_{i} g_{\theta}^{15}\right], & J_{v}=0 \\ \frac{1}{V_{i}}\left[-g_{\theta}^{15}(1-\sigma) J_{s}^{-}+J_{v} D J_{s}^{-}-J_{v}(1-\sigma) E+c_{i} g_{\theta}^{15}\right], & J_{v}<0\end{cases}
$$

Using (3.22) and (3.23), we solve the system (3.9) to obtain $\frac{\partial V_{p}}{\partial \theta_{k}}$ for $k=1, \ldots, 5$.

Then we plug-in these values to 3.20 to obtain the normalized sensitivities $s_{k}$. 
Figure 3.2 shows the graphs of the sensitivities of the model output $H c t(t)$ with respect to the parameters $a, L, d, b$ and $c$ at the nominal parameter $\theta_{0}$,

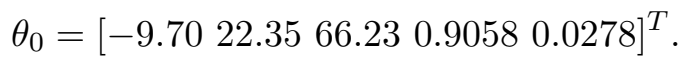

Plots of the sensitivities are shown for increasing values of CRP concentration. For most cases, $d$ is the least sensitive parameter, while $b$ is the most sensitive parameter. This means that $d$ has the least influence to the model output hematocrit on the time interval $[0,60]$ for values of $d$ close to the $d_{0}=66.23$. We also expect that $d$ can not be estimated with sufficient accuracy from hematocrit measurements taken during the first hour of HD. The opposite can be said for the parameter $b$ for values of $b$ close to $b_{0}=0.9058$. Among the five parameters, hematocrit measurements carry the most information about the parameter $b$. Hence, we expect that $b$ can be estimated with the most accuracy among the five parameters.

The sensitivity of the parameter $c$ is small for low concentrations of CRP but it begins to deviate from zero as CRP levels increase. We see in figure 3.2 that for CRP concentration equal to $0.020 \mathrm{mg} / \mathrm{mL}$, the sensitivities of the parameters $b$ and $c$ are almost the same. Aside from the parameter $b$, obtaining good estimates for $c$ may also be possible. We note that both parameters $b$ and $c$ appear in the function $\sigma([C R P])$.

The magnitudes of the sensitivities of the other two parameters $a$ and $L$ oscillate during the interval $[0,60]$. The trend is that their sensitivities start small and begin to increase, then approach zero for some $t \in[30,50]$ before increasing slightly again. This occurs except for the case when CRP concentration is $0.012 \mathrm{mg} / \mathrm{mL}$, where $s_{a}$ and $s_{L}$ remain close to zero during the last 20 minutes. Hence, we do not know for sure if $a$ and $L$ may be estimated accurately using measurements for hematocrit taken during $t \in[0,60]$.

For very high values of $\mathrm{CRP}$ concentration, $[C R P] \approx 0.025 \mathrm{mg} / \mathrm{mL}$ or greater, the sensitivities are large and become increasingly fluctuating until they fail to give information for the entire interval $[0,60]$. 

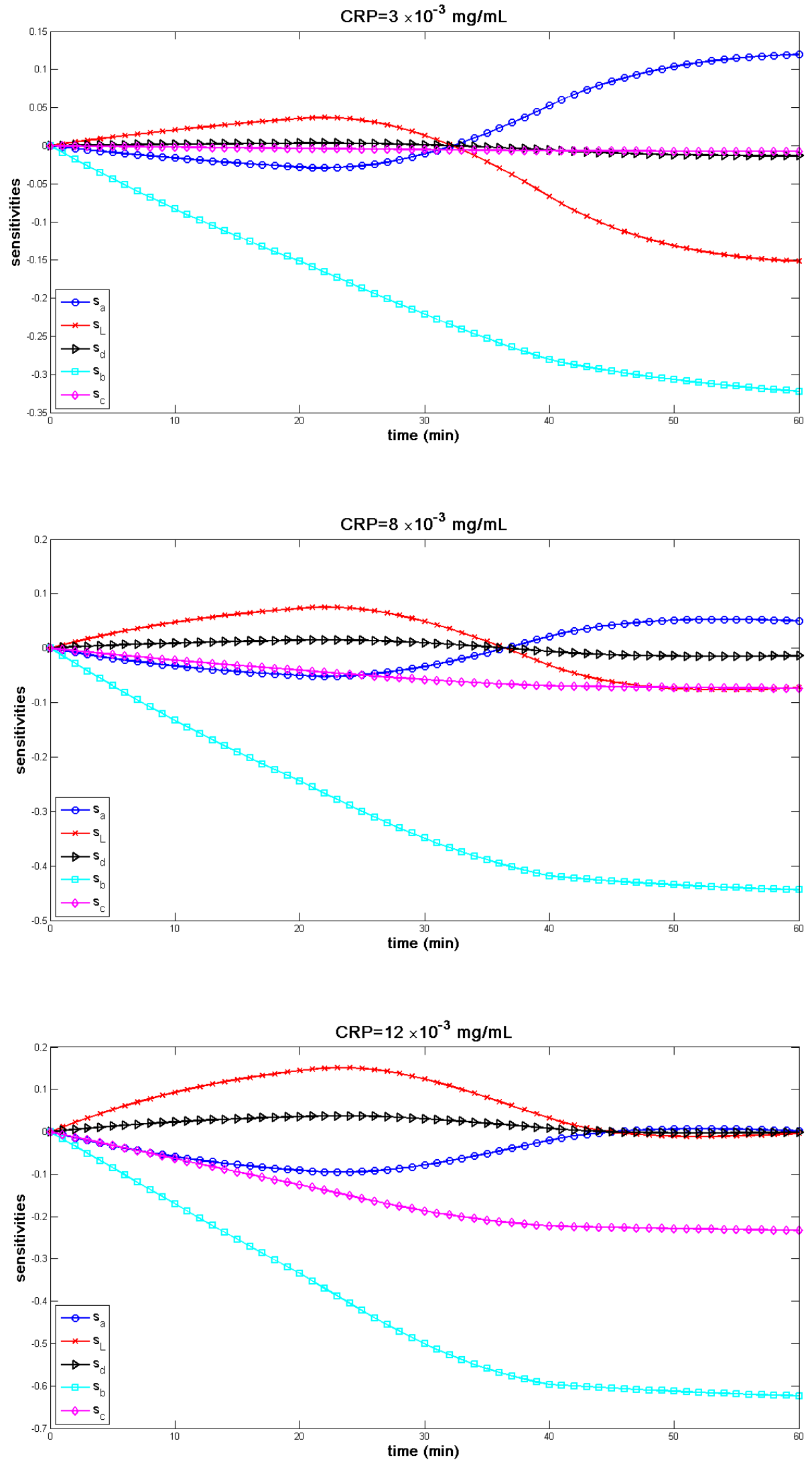

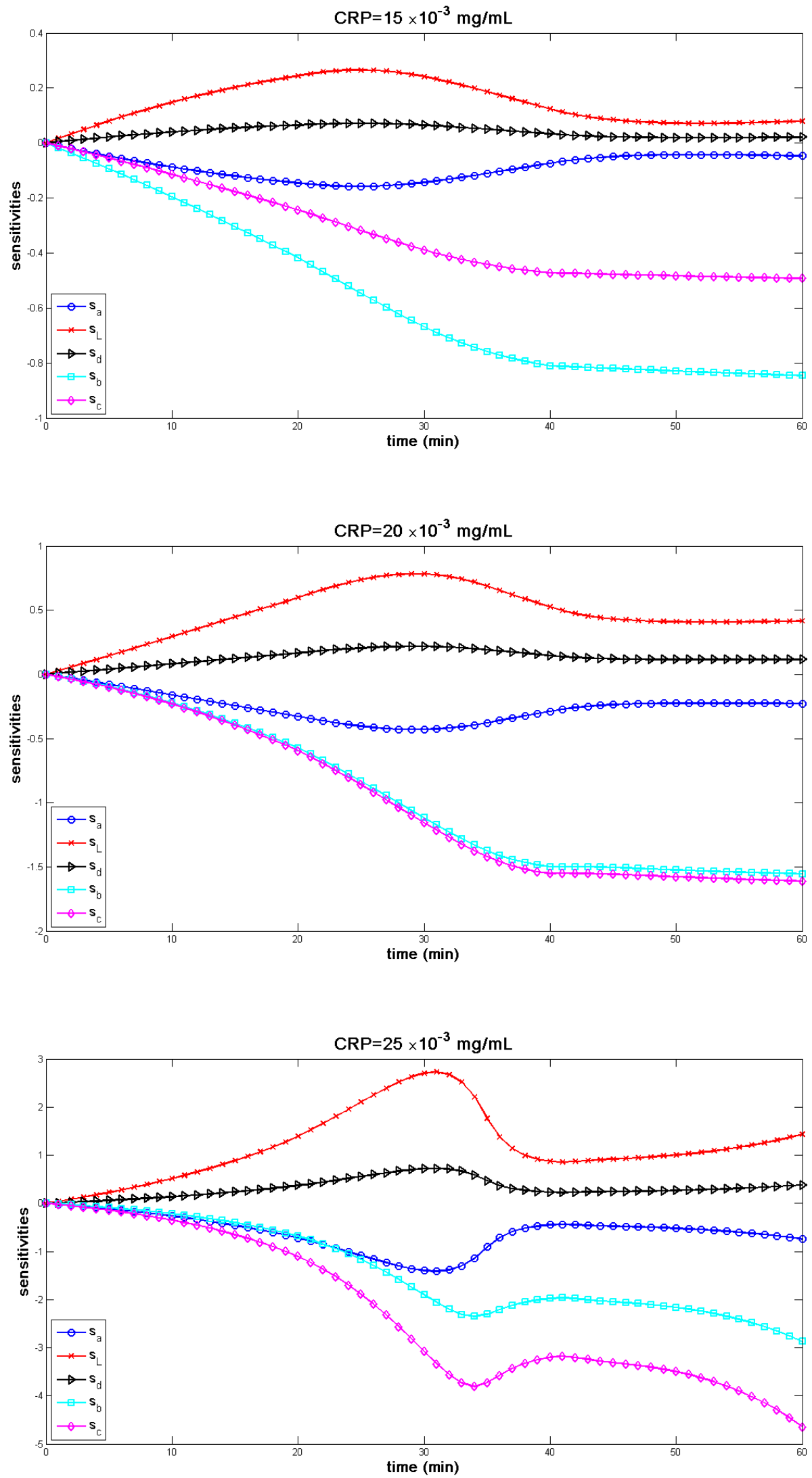

Figure 3.2: Sensitivities of hematocrit for different levels of CRP 


\subsubsection{Sensitivities with respect to fluid flux}

Now we consider the fluid flux $J_{v}$ as the model output and compute its sensitivities with respect to the parameters $a, L, d, b$, and $c$ at the same nominal parameter $\theta_{0}=$ [-9.70 22.35 66.23 0.9058 0.0278].

Recall from equation 2.6 that $J_{v}$ is given by

$$
J_{v}(t)=L_{p}([C R P])\left\{\sigma([C R P])\left(\pi_{p}(t)-\pi_{i}(t)\right)-\left(P_{c}-P_{i}\right)\right\},
$$

where

$$
\begin{aligned}
L_{p}([C R P]) & =a+\frac{L}{1+e^{-d[C R P]}} \\
\sigma([C R P]) & =b e^{-\left(\frac{[C R P]}{c}\right)^{2}} \\
\pi_{p}(t) & =a_{p, 1} c_{p}(t)+a_{p, 2} c_{p}(t)^{2} \\
\pi_{i}(t) & =a_{i, 1} c_{i}(t)+a_{i, 2} c_{i}(t)^{2} .
\end{aligned}
$$

Changing the model output from $H c t$ to $J_{v}$ requires some of the derivatives that we have already computed in the previous section. In addition, we only need to determine the corresponding expressions for $\operatorname{grad}_{x} h(t, x(t, \theta), \theta)$ and $\frac{\partial h}{\partial \theta_{k}}(t, x(t, \theta), \theta)$ for $k=1,2,3,4,5$, which appear in equation (3.7) because now we consider $h(t, x, \theta)=J_{v}(t, x, \theta)$. On the other hand, $y:=\frac{\partial x}{\partial \theta_{k}}(t, \theta)$ does not change and therefore, the system (3.9) also does not change. So, if $J_{v}$ is the model output and using the same notations for the derivatives as before, then we have

$$
\begin{aligned}
\operatorname{grad}_{x} h(t, x(t, \theta), \theta) & =\left[\begin{array}{llll}
0 & \frac{\partial J_{v}}{\partial c_{p}} & 0 & \frac{\partial J_{v}}{\partial c_{i}}
\end{array}\right]=\left[\begin{array}{llll}
0 & g_{x}^{12} & 0 & g_{x}^{14}
\end{array}\right] \\
\frac{\partial h}{\partial \theta_{k}}(t, x(t, \theta), \theta) & =\frac{\partial J_{v}}{\partial \theta_{k}}(t, x(t, \theta), \theta)=g_{\theta}^{1 k}, \quad \text { for } k=1,2,3,4,5 .
\end{aligned}
$$

Once the extended system (3.9) is solved, we compute for $\frac{\partial f}{\partial \theta_{k}}(t, \theta)$ in (3.7) using the solution $y$ and the expressions from (3.24) and (3.25). Following the discussion in section 3.1. for each $k$ we get

$$
\frac{\partial f}{\partial \theta_{k}}(t, \theta)=g_{x}^{12} \frac{\partial c_{p}}{\partial \theta_{k}}+g_{x}^{14} \frac{\partial c_{i}}{\partial \theta_{k}}+g_{\theta}^{1 k}
$$

The value of $J_{v}\left(t, x\left(t, \theta_{0}\right), \theta_{0}\right)$ can be calculated using the values in tables 2.6 and 2.8 . Finally, using the value of $J_{v}\left(t, x\left(t, \theta_{0}\right), \theta_{0}\right)$ and $(3.26), s_{k}\left(t, \theta_{0}\right)$ in $(3.6)$ can be computed for $0 \leq t \leq 60$.

Figure 3.4 shows the graphs of the sensitivities of the fluid flux with respect to the parameters $a, L, d, b$, and $c$ at the nominal parameter $\theta_{0}$. We initially recognize the spike in the sensitivities of all the parameters on the interval $[20,30]$ for CRP levels up to about 
$0.012 \mathrm{mg} / \mathrm{mL}$, and on the interval $[30,40]$ for CRP levels of about $0.018 \mathrm{mg} / \mathrm{mL}$ and above. We also observe that another spike in sensitivities develops as CRP levels increase further. A developing spike is already visible for $[C R P]=0.020 \mathrm{mg} / \mathrm{mL}$. Also, a second spike exists on the interval $[40,50]$ when $[C R P]=0.025 \mathrm{mg} / \mathrm{mL}$. The spikes tell us that $J_{v}$ has the most information about the parameters during the times when these spikes occur. For this reason, parameter estimation will later be done using measurements for $J_{v}$ taken on the interval $[20,40]$, when the spikes occur in most cases.

For CRP level less than $0.018 \mathrm{mg} / \mathrm{mL}$, the parameter $b$ has obviously the largest spike among the five and is therefore regarded as the most sensitive. Similar to the case when hematocrit is the model output, we expect that $b$ is the parameter that can be most easily estimated given measurements for $J_{v}$. For $[C R P] \approx 0.018 \mathrm{mg} / \mathrm{mL}$, it appears that besides $b$, the parameters $c$ and $L$ also become more sensitive.

To summarize, for CRP concentration less than $0.025 \mathrm{mg} / \mathrm{mL}$, measurements for both outputs $H c t$ and $J_{v}$ during the period $[0,60]$ seem to consist more information on the parameters related to the reflection coefficient $\sigma$, which are $b$ and $c$, rather than on the parameters related to the filtration coefficient $L_{p}$. We verify this hypothesis when we do parameter estimation in the following sections. 

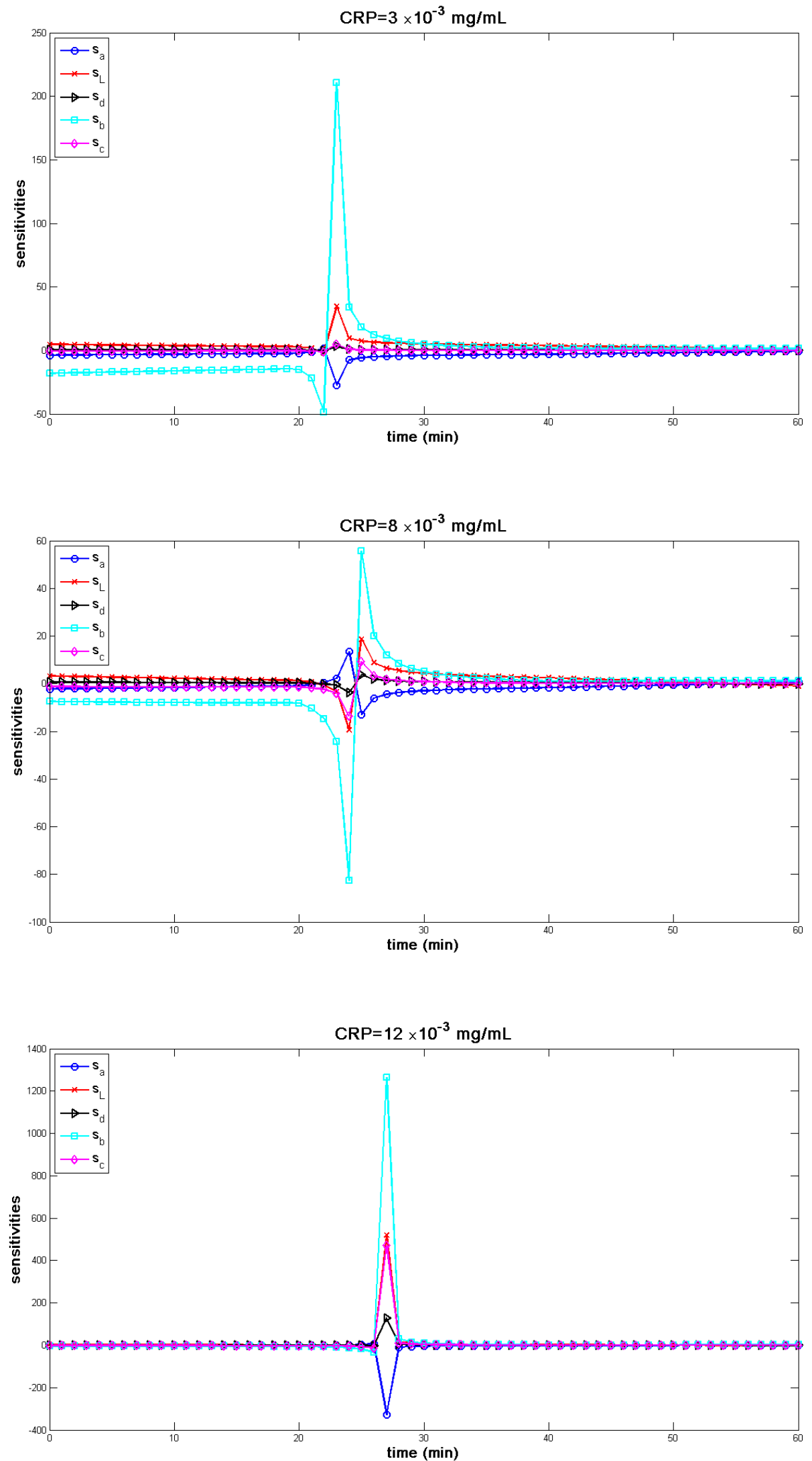

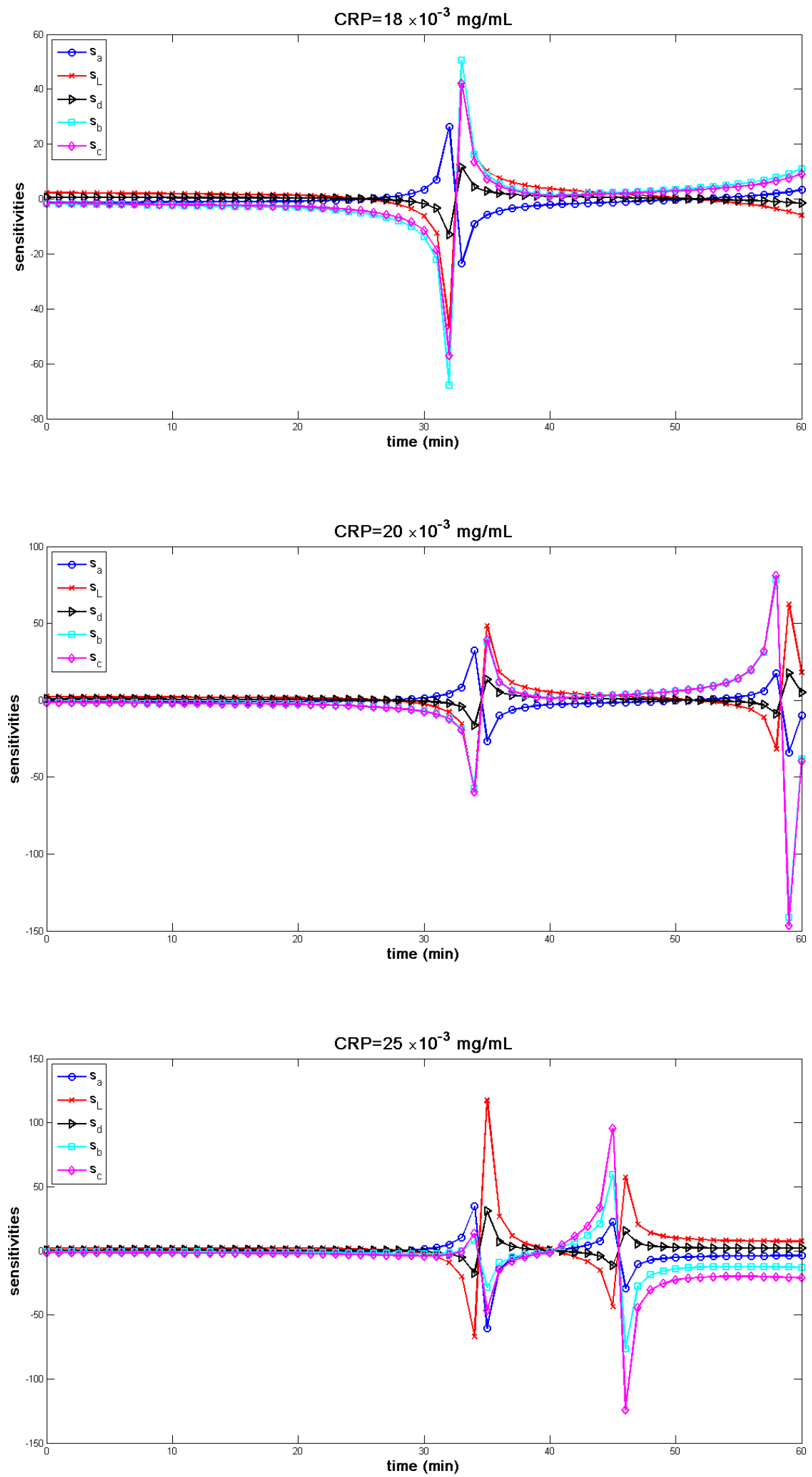

Figure 3.4: Sensitivities of $J_{v}$ for different levels of CRP 


\subsection{Subset selection results}

Before doing parameter estimation, we first implement the subset selection algorithm discussed earlier. We would like to determine which subsets of $\{a, L, d, b, c\}$ of lengths 2, 3 and 4 may give estimates with reliable accuracy using a measure based on standard errors.

Two cases are considered for the subset selection and parameter estimation, which differ on the level of CRP concentration. First, $[C R P]=0.00385 \mathrm{mg} / \mathrm{mL}$ to represent patients having a low level of inflammation; and second, $[C R P]=0.018 \mathrm{mg} / \mathrm{mL}$ to represent patients with a high level of inflammation. We refer to these cases as $C R P_{\text {low }}$ and $C R P_{\text {high }}$, respectively. Table 3.1 shows the initial values of the state variables for the two cases. The rest of the parameter values used in the simulations are in tables 2.6 and 2.8. The nominal variance is assumed to be 0.01 and the sampling times are assumed to be per second, uniformly spaced from $t=0$ to 60 minutes.

\begin{tabular}{|l|l|l|}
\hline Parameter & $C_{R P_{\text {low }}}$ & $C R P_{\text {high }}$ \\
\hline \hline$[C R P]$ & 0.00385 & 0.018 \\
$L_{p}$ & 2.8921 & 7.4452 \\
$\sigma$ & 0.8886 & 0.5956 \\
$V_{p}^{0}$ & 5352.5 & 5121.3 \\
$c_{p}^{0}$ & 73.5 & 73.5 \\
$V_{i}^{0}$ & 16753 & 17668 \\
$c_{i}^{0}$ & 24.5 & 24.5 \\
\hline
\end{tabular}

Table 3.1: Initial values for the cases $C R P_{\text {low }}$ and $C R P_{\text {high }}$

The basis for selecting the best parameter subset makes use of the asymptotic standard errors. We also look at the condition number of the sensitivity matrices of the corresponding subsets to give us a measure of how feasible the subsets are. Table 3.2 summarizes the results for the parameter vector $(a, L, d, b, c)$ considering the two model outputs $H c t$ and $J_{v}$, for the cases $C R P_{\text {low }}$ and $C R P_{\text {high }}$.

\begin{tabular}{|l|c|c|c|c|}
\hline Case & \multicolumn{2}{|c|}{$H c t$} & \multicolumn{2}{c|}{$J_{v}$} \\
\hline & $\kappa(\chi(\theta))$ & $\alpha(\theta)$ & $\kappa(\chi(\theta))$ & $\alpha(\theta)$ \\
\hline \hline$C R P_{\text {low }}$ & $8.6273 \times 10^{11}$ & $6.3178 \times 10^{7}$ & $\chi(\theta)$ does not have full rank \\
$C R P_{\text {high }}$ & $2.521 \times 10^{11}$ & $1.1593 \times 10^{6}$ & $3.1064 \times 10^{10}$ & 500.35 \\
\hline
\end{tabular}

Table 3.2: Selection scores of the parameter vector $(a, L, d, b, c)$ for $H c t$ and $J_{v}$ for $C R P_{\text {low }}$ and $C R P_{\text {high }}$ 
When hematocrit is the model output, the selection scores, as well as the condition numbers of the sensitivity matrix for the vector $(a, L, d, b, c)$ are very high in both cases. There is a high possibility of uncertainty for at least one of these parameters and the vector may not be reliably estimated. When we consider fluid flux as the model output, the sensitivity matrix for the case $C R P_{\text {low }}$ does not have full rank. Hence, we may not be able to estimate the vector $(a, L, d, b, c)$. For the case $C R P_{h i g h}$, the value of $\alpha$ is still high at 500.35, but this value is much lower compared to the others. This implies that using measurements for fluid flux, it is possible to get more reliable estimates for the vector $(a, L, d, b, c)$ compared to when hematocrit measurements are used in the estimation.

In the following subsections, we compute for the condition numbers of the sensitivity matrices $\kappa(\chi(\theta))$ and selection scores $\alpha(\theta)$ based on asymptotic standard errors for parameter subsets of lengths 2,3 , and 4 . We compare the results when hematocrit Hct or fluid flux $J_{v}$ are considered separately as the model outputs.

\subsubsection{Slightly elevated CRP concentration}

This subsection considers the case $C R P_{\text {low }}$, that is, $[C R P]=0.00385 \mathrm{mg} / \mathrm{mL}$. Tables 3.3 3.4 and 3.5 list the viable parameter subsets of lengths 4,3 , and 2 with selection scores arranged from least to greatest and hematocrit as the model output. Meanwhile, tables 3.6, 3.7, and 3.8 show the viable parameter subsets of lengths 4,3 , and 2 , with selection scores arranged from least to greatest and fluid flux as the model output.

We observe that in general, the computed selection scores become smaller as the length of the subsets decreases. Also, the selection scores are much smaller when $J_{v}$ is considered as the model output than when Hct is the model output. However, almost all of the condition numbers of the sensitivity matrices remain very large, except for parameter subsets of length 2 .

\begin{tabular}{|l|l|l|}
\hline \multicolumn{3}{|c|}{$\boldsymbol{C R P}_{\text {low }}: \boldsymbol{H} \boldsymbol{c t}$} \\
\hline parameter vector $\theta$ & $\kappa(\chi(\theta))$ & $\alpha(\theta)$ \\
\hline \hline$(a, L, b, c)$ & $1.0043 \times 10^{11}$ & $1.5044 \times 10^{7}$ \\
$(a, L, d, b)$ & $1.8491 \times 10^{11}$ & $6.2303 \times 10^{7}$ \\
$(L, d, b, c)$ & $5.7176 \times 10^{11}$ & $6.4038 \times 10^{7}$ \\
$(a, d, b, c)$ & $4.7894 \times 10^{11}$ & $7.7585 \times 10^{7}$ \\
$(a, L, d, c)$ & $2.3111 \times 10^{11}$ & $1.7776 \times 10^{8}$ \\
\hline
\end{tabular}

Table 3.3: Viable parameter vectors when $p=4,[C R P]=0.00385 \frac{\mathrm{mg}}{\mathrm{mL}}$, and Hct is the model output 


\begin{tabular}{|l|l|l|}
\hline \multicolumn{3}{|c|}{$\boldsymbol{C R P}_{\text {low }}:$ Hct } \\
\hline parameter vector $\theta$ & $\kappa(\chi(\theta))$ & $\alpha(\theta)$ \\
\hline \hline$(a, b, c)$ & $2.5344 \times 10^{5}$ & $1.1568 \times 10^{5}$ \\
$(L, b, c)$ & $2.5344 \times 10^{5}$ & $1.1568 \times 10^{5}$ \\
$(d, b, c)$ & $2.5345 \times 10^{5}$ & $1.1568 \times 10^{5}$ \\
$(a, L, c)$ & $7.0734 \times 10^{10}$ & $2.4351 \times 10^{7}$ \\
$(a, L, b)$ & $5.6599 \times 10^{10}$ & $3.6907 \times 10^{7}$ \\
$(a, L, d)$ & $2.4646 \times 10^{9}$ & $4.7145 \times 10^{7}$ \\
$(a, d, c)$ & $1.5621 \times 10^{11}$ & $5.2621 \times 10^{7}$ \\
$(a, d, b)$ & $1.2499 \times 10^{11}$ & $5.376 \times 10^{7}$ \\
$(L, d, b)$ & $1.3368 \times 10^{11}$ & $5.8361 \times 10^{7}$ \\
$(L, d, c)$ & $1.6707 \times 10^{11}$ & $6.1518 \times 10^{7}$ \\
\hline
\end{tabular}

Table 3.4: Viable parameter vectors when $p=3,[C R P]=0.00385 \frac{\mathrm{mg}}{\mathrm{mL}}$, and $H c t$ is the model output

\begin{tabular}{|l|l|l|}
\hline \multicolumn{3}{|c|}{$\boldsymbol{C R P}_{\text {low }}:$ Hct } \\
\hline parameter vector $\theta$ & $\kappa(\chi(\theta))$ & $\alpha(\theta)$ \\
\hline \hline$(L, b)$ & 151.51 & 0.22411 \\
$(a, b)$ & 85.362 & 0.28888 \\
$(L, c)$ & 189.35 & 1.1091 \\
$(a, c)$ & 106.68 & 1.124 \\
$(d, b)$ & 4032.8 & 1.9784 \\
$(d, c)$ & 5040.2 & 2.257 \\
$(b, c)$ & $2.4252 \times 10^{5}$ & $1.1069 \times 10^{5}$ \\
$(a, L)$ & $9.363 \times 10^{7}$ & $1.1363 \times 10^{7}$ \\
$(a, d)$ & $5.1983 \times 10^{8}$ & $1.8533 \times 10^{7}$ \\
$(L, d)$ & $3.9791 \times 10^{8}$ & $2.5593 \times 10^{7}$ \\
\hline
\end{tabular}

Table 3.5: Viable parameter vectors when $p=2,[C R P]=0.00385 \frac{m g}{m L}$, and $H c t$ is the model output

When hematocrit is considered as the model output, the parameter vectors of lengths 3 and 4 all have very large selection scores and condition numbers. For parameter subsets of length $2, \alpha(\theta)$ and $\kappa(\chi(\theta))$ are greatly reduced for some but not all of the subsets. When $p=4$, the best subset is $(a, L, b, c)$ with $\alpha(\theta)=1.5044 \times 10^{7}$ and for $p=3$, the best subsets are $(a, b, c),(L, b, c)$, and $(d, b, c)$ with $\alpha(\theta)=1.1568 \times 10^{5}$. Interestingly, there are three best parameters when $p=3$ and they are combinations of either $a, L$, or $d$ from the function $L_{p}([C R P])$, plus the two parameters $b$ and $c$ from the function $\sigma([C R P])$. When $p=2$, the best parameter subset is $(L, b)$ with selection score 0.22411 . 
Most subsets in this case have small $\alpha$ but there still exist four subsets with very high selection scores and one of these four is the subset $(b, c)$. This shows us that although the pair $b, c$ appears in the best parameter subsets for $p=3$ and 4 , the combination of just $(b, c)$ does not necessarily give a good score.

The next tables are results when $J_{v}$ is the model output. We see that although selection scores based on standard errors are significantly reduced, the condition numbers of the sensitivity matrices remain very large.

\begin{tabular}{|l|l|l|}
\hline \multicolumn{3}{|c|}{$\boldsymbol{C R P}_{\text {low }}: \boldsymbol{J}_{\boldsymbol{v}}$} \\
\hline parameter vector $\theta$ & $\kappa(\chi(\theta))$ & $\alpha(\theta)$ \\
\hline \hline$(a, L, b, c)$ & $1.3301 \times 10^{10}$ & 18818 \\
$(a, d, b, c)$ & $3.5604 \times 10^{11}$ & 54979 \\
$(L, d, b, c)$ & $1.8743 \times 10^{12}$ & 79317 \\
$(a, L, d, c)$ & $1.1676 \times 10^{12}$ & 81288 \\
$(a, L, d, b)$ & $9.3424 \times 10^{11}$ & $1.93 \times 10^{5}$ \\
\hline
\end{tabular}

Table 3.6: Viable parameter vectors when $p=4,[C R P]=0.00385 \frac{m g}{m L}$, and $J_{v}$ is the model output

\begin{tabular}{|l|l|l|}
\hline \multicolumn{3}{|c|}{$\boldsymbol{C R P}_{\boldsymbol{l o w}}: \boldsymbol{J}_{\boldsymbol{v}}$} \\
\hline parameter vector $\theta$ & $\kappa(\chi(\theta))$ & $\alpha(\theta)$ \\
\hline \hline$(a, L, c)$ & $7.3795 \times 10^{9}$ & 12843 \\
$(a, b, c)$ & $2.2304 \times 10^{7}$ & 13737 \\
$(L, b, c)$ & $2.2304 \times 10^{7}$ & 13737 \\
$(d, b, c)$ & $2.2304 \times 10^{7}$ & 13737 \\
$(a, L, b)$ & $5.9045 \times 10^{9}$ & 14335 \\
$(L, d, b)$ & $9.3302 \times 10^{11}$ & 45057 \\
$(a, d, b)$ & $1.3649 \times 10^{11}$ & 45246 \\
$(a, d, c)$ & $1.7058 \times 10^{11}$ & 45246 \\
$(L, d, c)$ & $1.1661 \times 10^{12}$ & 63721 \\
$(a, L, d)$ & $3.9362 \times 10^{10}$ & inf \\
\hline
\end{tabular}

Table 3.7: Viable parameter vectors when $p=3,[C R P]=0.00385 \frac{m g}{m L}$, and $J_{v}$ is the model output

First, we observe that the best parameter subsets of lengths 4 and 2 are $(a, L, b, c)$ and $(L, b)$, respectively, which are the same as the results using $H c t$ as the model output. Second, we see a different result for the case when $p=3$, where we obtain the best parameter subset $(a, L, c)$ with $\alpha(\theta)=12843$. But we also notice from table 3.7 that the 


\begin{tabular}{|l|l|l|}
\hline \multicolumn{3}{|c|}{$\boldsymbol{C R}_{\text {low }}: \boldsymbol{J}_{\boldsymbol{v}}$} \\
\hline parameter vector $\theta$ & $\kappa(\chi(\theta))$ & $\alpha(\theta)$ \\
\hline \hline$(L, b)$ & 47.523 & 0.00010621 \\
$(d, b)$ & 1265 & 0.00084322 \\
$(L, c)$ & 59.395 & 0.001307 \\
$(a, c)$ & 33.465 & 0.0013093 \\
$(a, b)$ & 26.776 & 0.00013152 \\
$(d, c)$ & 1581 & 0.0015518 \\
$(b, c)$ & $2.0226 \times 10^{6}$ & 1252.5 \\
$(a, L)$ & $1.7206 \times 10^{8}$ & 9609.9 \\
$(a, d)$ & $3.6066 \times 10^{9}$ & 45246 \\
$(L, d)$ & $1.7925 \times 10^{10}$ & 63721 \\
\hline
\end{tabular}

Table 3.8: Viable parameter vectors when $p=2,[C R P]=0.00385 \frac{m g}{m L}$, and $J_{v}$ is the model output

three best parameters from the hematocrit case come second best with $\alpha(\theta)=13737$. Finally, when $p=2$, the selection scores are small for most but not all subsets. There are four subsets with remarkably higher scores than the rest of the subsets. The four subsets, which are $(b, c),(a, L),(a, d)$, and $(L, d)$, are the same four subsets which obtained the largest scores when Hct is the model output.

\subsubsection{High CRP concentration}

This subsection considers the case $C R P_{h i g h}$, when $[C R P]=0.018 \mathrm{mg} / \mathrm{mL}$. Tables 3.9 . 3.10, and 3.11 show the viable parameter subsets when hematocrit is considered as the model output, while tables 3.12 , 3.13 , and 3.14 show the viable parameter subsets when fluid flux is considered as the model output.

\begin{tabular}{|l|l|l|}
\hline \multicolumn{3}{|c|}{$\boldsymbol{C R P}_{\text {high }}: \boldsymbol{H} \boldsymbol{c t}$} \\
\hline parameter vector $\theta$ & $\kappa(\chi(\theta))$ & $\alpha(\theta)$ \\
\hline \hline$(a, L, b, c)$ & $1.9631 \times 10^{10}$ & $3.5288 \times 10^{5}$ \\
$(a, L, d, c)$ & $6.6007 \times 10^{10}$ & $4.3877 \times 10^{5}$ \\
$(a, L, d, b)$ & $2.4168 \times 10^{9}$ & $4.4179 \times 10^{5}$ \\
$(L, d, b, c)$ & $1.4916 \times 10^{11}$ & $5.9023 \times 10^{5}$ \\
$(a, d, b, c)$ & $2.4956 \times 10^{11}$ & $1.1021 \times 10^{6}$ \\
\hline
\end{tabular}

Table 3.9: Viable parameter vectors when $p=4,[C R P]=0.018 \frac{m g}{m L}$, and Hct is the model output 


\begin{tabular}{|l|l|l|}
\hline \multicolumn{3}{|c|}{$\boldsymbol{C R}_{\boldsymbol{h i g h}} \boldsymbol{:} \boldsymbol{H c t}$} \\
\hline parameter vector $\theta$ & $\kappa(\chi(\theta))$ & $\alpha(\theta)$ \\
\hline \hline$(a, b, c)$ & $1.4604 \times 10^{7}$ & 6201.9 \\
$(L, b, c)$ & $1.4604 \times 10^{7}$ & 6201.9 \\
$(d, b, c)$ & $1.4604 \times 10^{7}$ & 6201.9 \\
$(L, d, b)$ & $2.3256 \times 10^{9}$ & $2.4475 \times 10^{5}$ \\
$(L, d, c)$ & $6.3528 \times 10^{10}$ & $2.4454 \times 10^{5}$ \\
$(a, d, b)$ & $2.4053 \times 10^{9}$ & $2.7253 \times 10^{5}$ \\
$(a, d, c)$ & $6.57 \times 10^{10}$ & $2.7274 \times 10^{5}$ \\
$(a, L, c)$ & $1.957 \times 10^{10}$ & $3.4692 \times 10^{5}$ \\
$(a, L, b)$ & $7.1657 \times 10^{8}$ & $3.4968 \times 10^{5}$ \\
$(a, L, d)$ & $4.3503 \times 10^{7}$ & $3.5932 \times 10^{5}$ \\
\hline
\end{tabular}

Table 3.10: Viable parameter vectors when $p=3,[C R P]=0.018 \frac{\mathrm{mg}}{\mathrm{mL}}$, and Hct is the model output

\begin{tabular}{|l|l|l|}
\hline \multicolumn{3}{|c|}{$\boldsymbol{C R}_{\boldsymbol{h i g h}}:$ Hct } \\
\hline parameter vector $\theta$ & $\kappa(\chi(\theta))$ & $\alpha(\theta)$ \\
\hline \hline$(L, b)$ & 140.32 & 0.045947 \\
$(L, c)$ & 3832.7 & 0.04735 \\
$(a, b)$ & 107.66 & 0.077055 \\
$(a, c)$ & 2940.1 & 0.077899 \\
$(d, b)$ & 1497.4 & 0.15388 \\
$(d, c)$ & 40910 & 0.15431 \\
$(b, c)$ & $8.4451 \times 10^{6}$ & 3586.5 \\
$(L, d)$ & $7.3592 \times 10^{6}$ & 45976 \\
$(a, d)$ & $1.2436 \times 10^{7}$ & 64196 \\
$(a, L)$ & $5.7568 \times 10^{6}$ & $1.0157 \times 10^{5}$ \\
\hline
\end{tabular}

Table 3.11: Viable parameter vectors when $p=2,[C R P]=0.018 \frac{\mathrm{mg}}{\mathrm{mL}}$, and $H c t$ is the model output

When hematocrit is considered as the model output and for the case $C R P_{\text {high }}$, we observe that the obtained best parameter subsets of lengths 4,3 , and 2 are the same as those obtained for the case $C R P_{\text {low }}$. Although the selection scores and condition numbers are lower in this case than in $C R P_{\text {low }}$, their values remain quite high.

The same observation regarding the selection scores with respect to the two outputs can be said for the case $C R P_{h i g h}$. When $J_{v}$ is considered as the model output, the best parameter subset of length 4 is $(a, d, b, c)$, which has the worst selection score when 


\begin{tabular}{|l|l|l|}
\hline \multicolumn{3}{|c|}{$\boldsymbol{C R P}_{\boldsymbol{h i g h}}: \boldsymbol{J}_{\boldsymbol{v}}$} \\
\hline parameter vector $\theta$ & $\kappa(\chi(\theta))$ & $\alpha(\theta)$ \\
\hline \hline$(a, d, b, c)$ & $2.7282 \times 10^{10}$ & 264.05 \\
$(L, d, b, c)$ & $3.0538 \times 10^{10}$ & 273.84 \\
$(a, L, d, c)$ & $2.982 \times 10^{10}$ & 291.97 \\
$(a, L, d, b)$ & $1.0917 \times 10^{9}$ & 292.1 \\
$(a, L, b, c)$ & $9.7118 \times 10^{9}$ & 409.69 \\
\hline
\end{tabular}

Table 3.12: Viable parameter vectors when $p=4,[C R P]=0.018 \frac{m g}{m L}$, and $J_{v}$ is the model output

\begin{tabular}{|l|l|l|}
\hline \multicolumn{3}{|c|}{$\boldsymbol{C R P}_{\boldsymbol{h i g h}}: \boldsymbol{J}_{\boldsymbol{v}}$} \\
\hline parameter vector $\theta$ & $\kappa(\chi(\theta))$ & $\alpha(\theta)$ \\
\hline \hline$(a, b, c)$ & $2.3408 \times 10^{7}$ & 23.075 \\
$(L, b, c)$ & $2.3408 \times 10^{7}$ & 23.075 \\
$(d, b, c)$ & $2.3408 \times 10^{7}$ & 23.076 \\
$(a, L, c)$ & $2.9656 \times 10^{9}$ & 122.86 \\
$(a, L, b)$ & $1.0857 \times 10^{8}$ & 122.92 \\
$(a, d, b)$ & $8.1875 \times 10^{8}$ & 215.52 \\
$(a, d, c)$ & $2.2365 \times 10^{10}$ & 215.52 \\
$(a, L, d)$ & $4.2686 \times 10^{7}$ & 250.35 \\
$(L, d, b)$ & $1.0467 \times 10^{9}$ & 256.11 \\
$(L, d, c)$ & $2.8594 \times 10^{10}$ & 256.31 \\
\hline
\end{tabular}

Table 3.13: Viable parameter vectors when $p=3,[C R P]=0.018 \frac{m g}{m L}$, and $J_{v}$ is the model output

hematocrit is the model output. Conversely, the subset with the worst score when $J_{v}$ is the model output is $(a, L, b, c)$, which has the best score when Hct is the model output. When $p=3$, we get the three best subsets which are the same as before. They are $(a, b, c),(L, b, c)$, and $(d, b, c)$ with score $\alpha=23.075$. When $p=2$, the best subset is still $(L, b)$ with $\alpha=3.4326 \times 10^{-5}$ followed very closely by $(L, c)$ with $\alpha=3.6531 \times 10^{-5}$.

Table 3.15 summarizes the best parameter subsets for each case and for each model output based on the selection score involving asymptotic standard errors. We see that the subsets having the best scores may differ when we consider different model outputs. One set of measurements may provide reliable estimates for a set of parameters, but another set of measurements may not give good estimates for the same set of parameters. In the next section, we do parameter estimation for some of these subsets. 


\begin{tabular}{|l|l|l|}
\hline \multicolumn{3}{|c|}{$\boldsymbol{R P}_{\boldsymbol{h i g h}}: \boldsymbol{J}_{\boldsymbol{v}}$} \\
\hline parameter vector $\theta$ & $\kappa(\chi(\theta))$ & $\alpha(\theta)$ \\
\hline \hline$(L, b)$ & 40.489 & $3.4326 \times 10^{-5}$ \\
$(L, c)$ & 1106 & $3.6531 \times 10^{-5}$ \\
$(a, b)$ & 31.063 & $5.3808 \times 10^{-5}$ \\
$(a, c)$ & 848.42 & $5.5241 \times 10^{-5}$ \\
$(d, b)$ & 432.11 & 0.0001042 \\
$(d, c)$ & 11805 & 0.00010495 \\
$(b, c)$ & $1.9476 \times 10^{7}$ & 19.201 \\
$(a, L)$ & $3.4891 \times 10^{6}$ & 87.667 \\
$(L, d)$ & $1.9847 \times 10^{7}$ & 176.61 \\
$(a, d)$ & $2.9302 \times 10^{7}$ & 215.33 \\
\hline
\end{tabular}

Table 3.14: Viable parameter vectors when $p=2,[C R P]=0.018 \frac{\mathrm{mg}}{\mathrm{mL}}$, and $J_{v}$ is the model output

\begin{tabular}{|c|c|c|c|c|c|c|}
\hline \multicolumn{7}{|c|}{$H c t$ as model output } \\
\hline Case & \multicolumn{2}{|r|}{$p=2$} & \multicolumn{2}{|c|}{$p=3$} & \multicolumn{2}{|c|}{$p=4$} \\
\hline & $\theta$ & $\alpha(\theta)$ & $\theta$ & $\alpha(\theta)$ & $\theta$ & $\alpha(\theta)$ \\
\hline$C R P_{\text {low }}$ & $(L, b)$ & 0.22411 & $\begin{array}{l}(a, b, c) \\
(L, b, c) \\
(d, b, c)\end{array}$ & $1.1568 \times 10^{5}$ & $(a, L, b, c)$ & $1.5044 \times 10^{7}$ \\
\hline$C R P_{h i g h}$ & $\begin{array}{l}(L, b) \\
(L, c)\end{array}$ & $\begin{array}{c}0.045947 \\
0.04735\end{array}$ & $\begin{array}{l}(a, b, c) \\
(L, b, c) \\
(d, b, c)\end{array}$ & 6201.9 & $(a, L, b, c)$ & $3.5288 \times 10^{5}$ \\
\hline \multicolumn{7}{|c|}{$J v$ as model output } \\
\hline Case & & $p=2$ & & $p=3$ & & $=4$ \\
\hline & $\theta$ & $\alpha(\theta)$ & $\theta$ & $\alpha(\theta)$ & $\theta$ & $\alpha(\theta)$ \\
\hline$C R P_{\text {low }}$ & $(L, b)$ & 0.00010621 & $(a, L, c)$ & 12843 & $(a, L, b, c)$ & 18818 \\
\hline$C R P_{\text {high }}$ & $\begin{array}{l}(L, b) \\
(L, c)\end{array}$ & $\begin{array}{l}3.4326 \times 10^{-5} \\
3.6531 \times 10^{-5}\end{array}$ & $\begin{array}{l}(a, b, c) \\
(L, b, c) \\
(d, b, c)\end{array}$ & 23.075 & $(a, d, b, c)$ & 264.05 \\
\hline
\end{tabular}

Table 3.15: Best parameter subsets of lengths 2,3 and 4 based on the selection score $\alpha(\theta)$ for the model outputs $H c t$ and $J_{v}$ 


\subsection{Estimation of best parameter subsets}

In this section, we would like to investigate how reliably the parameters $a, L, d, b$, and $c$ can be estimated from measurements of hematocrit and fluid flux on a subinterval of $[0,60]$ minutes. We again consider the two cases $C R P_{\text {low }}$ and $C R P_{\text {high }}$, where $[C R P]=$ 0.00385 and $0.018 \mathrm{mg} / \mathrm{mL}$, respectively. Estimates of the best parameter subsets of lengths 2,3 , and 4 , according to the results in table 3.15 are presented.

Before doing parameter estimation, we need measurement outputs. Since real hematocrit and fluid flux measurements related to this study are not available at the moment, synthetic data are generated as described in 86. The measurements $y_{j}$, which are representations of the random variable $\mathcal{Y}_{j}$, are produced by adding noise to the nominal model output $f\left(t_{j}, \theta_{0}\right)$ in the following way

$$
\mathcal{Y}_{j}=f\left(t_{j}, \theta_{0}\right)+\underbrace{\sigma_{0} \mathcal{V}_{j}}_{\text {noise }}, \quad j=1, \ldots, N,
$$

where $\mathcal{V}_{j}$ is a standard normal random variable and $\sigma_{0}$ is the standard deviation. In the simulations, the noise is generated using the function randn in Matlab, such that it is normally distributed with mean zero and variance $\sigma_{0}^{2}=0.01$. The synthetic data is generated for the period $[0,60]$ minutes with frequency of measurements set to be 60 times per minute. Since we have generated the measurements in this way, then we say that the parameters can be estimated with sufficient accuracy if the estimates

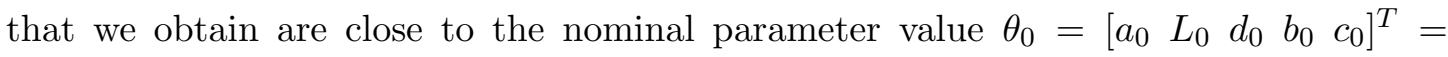

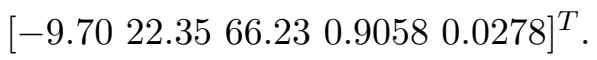

For the model output hematocrit, the estimates are calculated using the measurements for $t \in[10,50]$ minutes. But for the model output $J_{v}$, the estimates are done using the measurements on the interval $[20,40]$ minutes. Here, we utilize the results from the sensitivity analysis. More information regarding the parameters may be provided by the fluid flux measurements taken during the period [20,40] minutes. Before we calculate estimates for the subsets, we first look at the estimates for the five parameters using measurements from the two outputs.

Tables 3.16 and 3.17 show the estimates for the five parameters when hematocrit is used as the model output for the cases $C R P_{\text {low }}$ and $C R P_{\text {high }}$. Table 3.18 shows the estimates for the five parameters for the case $C R P_{h i g h}$ when $J_{v}$ is used as the model output. The Matlab function fminsearch is used to obtain the estimates for the parameters. The values on the left column are the starting values for the search, while the values on the right column are the obtained estimates.

Firstly, we observe that in some cases, we obtain estimates for $b$ that are greater than one. This should not be the case since $b$ is a parameter that appears in the function for 


\begin{tabular}{|l|l|}
\hline \multicolumn{2}{|c|}{$\boldsymbol{C R P}_{\text {low }}:$ Hct } \\
\hline starting values $(a, L, d, b, c)$ & estimated values \\
\hline \hline$(-10,22,60,0.91,0.03)$ & $(-9.8454,22.808,61.625,0.90314,0.029703)$ \\
$(-8,25,73,0.88,0.015)$ & $(-9.9081,22.593,70.156,0.94143,0.015942)$ \\
$(-11.5,19,62,0.9,0.01)$ & $(-9.8898,22.747,65.259,1.0246,0.010182)$ \\
$(-11.8,27,71,0.89,0.04)$ & $(-12.147,26.531,70.382,0.8961,0.040637)$ \\
$(-7,18,80,0.78,0.01)$ & $(-9.489,22.897,43.005,1.1172,0.0080367)$ \\
\hline
\end{tabular}

Table 3.16: Estimates for $(a, L, d, b, c)$ when $[C R P]=0.00385 \mathrm{mg} / \mathrm{mL}$ and $H c t$ is the model output

\begin{tabular}{|l|l|}
\hline \multicolumn{2}{|c|}{$\boldsymbol{C R P}_{\text {high }}:$ Hct } \\
\hline starting values $(a, L, d, b, c)$ & estimated values \\
\hline \hline$(-10,22,60,0.91,0.03)$ & $(-9.9325,23.024,62.461,0.85882,0.029749)$ \\
$(-11.5,19,62,0.9,0.01)$ & $(-13.364,20.344,61.381,1.0696,0.011896)$ \\
$(-11.8,27,71,0.89,0.04)$ & $(-13.212,25.05,86.011,0.73085,0.039781)$ \\
$(-7,18,80,0.78,0.01)$ & $(-6.8933,19.235,81.399,0.77778,0.0097261)$ \\
$(-8,25,73,0.88,0.02)$ & $(-8.5756,20.421,71.802,0.94207,0.02658)$ \\
\hline
\end{tabular}

Table 3.17: Estimates for $(a, L, d, b, c)$ when $[C R P]=0.018 \mathrm{mg} / \mathrm{mL}$ and $H c t$ is the model output

\begin{tabular}{|l|l|}
\hline \multicolumn{2}{|l|}{$\boldsymbol{C R P}_{\boldsymbol{h i g h}}: \boldsymbol{J}_{\boldsymbol{v}}$} \\
\hline starting values $(a, L, d, b, c)$ & estimated values \\
\hline \hline$(-10,22,60,0.91,0.03)$ & $(-9.9324,22.9712,62.9621,0.8661,0.0294)$ \\
$(-11.5,19,62,0.9,0.01)$ & $(-14.672,80.245,-53.685,0.82844,0.031337)$ \\
$(-11.8,27,71,0.89,0.04)$ & $(-13.667,26.767,73.168,0.71917,0.04146)$ \\
$(-7,18,80,0.78,0.01)$ & $(11.205,-3.7682,346.11,2.7169,0.014611)$ \\
$(-8,25,73,0.88,0.02)$ & $(-8.6338,21.814,57.262,1.0325,0.024269)$ \\
\hline
\end{tabular}

Table 3.18: Estimates for $(a, L, d, b, c)$ when $[C R P]=0.018 \mathrm{mg} / \mathrm{mL}$ and $J_{v}$ is the model output

$\sigma$, which is restricted to have a value of at most 1 . Secondly, we notice that sometimes we obtain relatively good estimates for some parameters, but not so good for the other parameters. For instance in table 3.16 for the case $C R P_{\text {low }}$, it seems that $a$ and $L$ can be estimated since most of the the values seem close to the nominal values for $a$ and $L$. However, if we consider the rest of the parameters, then we cannot conclude that $(a, L, d, b, c)$ can be estimated reliably. We see from tables 3.17 and 3.18 that the estimates lie in a quite large range of values. For these reasons, we do estimation for some parameter subsets in table 3.15 and see whether better estimates can be obtained. 


\subsubsection{Slightly elevated CRP concentration}

In this part, we present estimates for some of the parameter subsets appearing in table 3.15 when $[C R P]=0.00385 \mathrm{mg} / \mathrm{mL}$. Tables 3.19 , 3.20, and 3.21 show the estimates when hematocrit is used as the model output for the parameter subsets $(a, L, b, c)$, $(L, b, c)$, and $(L, b)$. We present in tables $3.22,3.23$, and 3.24 the estimates when $J_{v}$ is the model output for the subsets $(a, L, b, c),(a, L, c)$, and $(L, b)$.

\begin{tabular}{|l|l|}
\hline \multicolumn{2}{|c|}{$\boldsymbol{C R P}_{\text {low }}: \boldsymbol{H c t}$} \\
\hline starting values $(a, L, b, c)$ & estimated values \\
\hline \hline$(-10,22,0.91,0.03)$ & $(-9.8185,22.583,0.90252,0.030328)$ \\
$(-8,25,0.88,0.015)$ & $(-8.1129,23.358,0.93533,0.015775)$ \\
$(-11.5,19,0.9,0.01)$ & $(-8.8386,20.844,1.0274,0.010084)$ \\
$(-11.8,27,0.89,0.04)$ & $(-12.114,26.657,0.89632,0.040101)$ \\
$(-7,18,0.78,0.01)$ & $(-4.9055,13.863,0.94962,0.014876)$ \\
\hline
\end{tabular}

Table 3.19: Estimates for $(a, L, b, c)$ when $[C R P]=0.00385 \mathrm{mg} / L$ and $H c t$ as the model output

\begin{tabular}{|l|l|}
\hline \multicolumn{2}{|c|}{$\boldsymbol{C R P}_{\text {low }}:$ Hct } \\
\hline starting values $(L, b, c)$ & estimated values \\
\hline \hline$(22,0.91,0.03)$ & $(22.373,0.90222,0.030643)$ \\
$(25,0.88,0.015)$ & $(22.373,0.93384,0.017179)$ \\
$(19,0.9,0.01)$ & $(22.373,0.99887,0.011229)$ \\
$(27,0.89,0.04)$ & $(22.373,0.89583,0.041328)$ \\
$(18,0.78,0.01)$ & $(22.373,2.9048,0.0035)$ \\
\hline
\end{tabular}

Table 3.20: Estimates for $(L, b, c)$ when $[C R P]=0.00385 \mathrm{mg} / L$ and $H c t$ as the model output

We observe from table 3.19 that if we consider different starting values for the fourparameter subset $(a, L, b, c)$, we still obtain estimates that lie on a wide range of values. Although the cost functional $J$ gives the same minimum value for most of the estimates after the search, the estimates do not converge to a single point. Some improvement on the estimates can be observed in table 3.20 , when the parameter subset $(L, b, c)$ is considered. We see that the parameter $L$ is locally identifiable but the other two parameters, $b$ and $c$, are not. Finally, table 3.21 shows that the two parameters $L$ and $b$ can 


\begin{tabular}{|l|l|}
\hline \multicolumn{2}{|c|}{$\boldsymbol{C R P}_{\text {low }}: \boldsymbol{H} \boldsymbol{c t}$} \\
\hline starting values $(L, b)$ & estimated values \\
\hline \hline$(22,0.91)$ & $(22.373,0.90529)$ \\
$(25,0.88)$ & $(22.373,0.90529)$ \\
$(19,0.9)$ & $(22.373,0.90529)$ \\
$(27,0.89)$ & $(22.373,0.90529)$ \\
$(18,0.78)$ & $(22.373,0.90529)$ \\
\hline
\end{tabular}

Table 3.21: Estimates for $(L, b)$ when $[C R P]=0.00385 \mathrm{mg} / L$ and $H c t$ as the model output

be estimated reliably from different starting values using the generated measurements for hematocrit. The parameter $L$ converges to 22.373 and $b$ converges to 0.90529 , which are both not far from their nominal values of 22.35 and 0.9058 , respectively.

\begin{tabular}{|l|l|}
\hline \multicolumn{2}{|c|}{$\boldsymbol{C R P}_{\text {low }}: \boldsymbol{J}_{\boldsymbol{v}}$} \\
\hline starting values $(a, L, b, c)$ & estimated values \\
\hline \hline$(-10,22,0.91,0.03)$ & $(-9.9772,22.838,0.90262,0.030955)$ \\
$(-8,25,0.88,0.015)$ & $(-8.7337,20.631,0.94499,0.015546)$ \\
$(-11.8,27,0.89,0.04)$ & $(-12.132,26.662,0.89669,0.040861)$ \\
$(-7,18,0.78,0.01)$ & $(-6.4077,16.502,0.99181,0.011624)$ \\
$(-11.5,20,0.85,0.02)$ & $(-9.3528,21.73,0.92104,0.020386)$ \\
\hline
\end{tabular}

Table 3.22: Estimates for $(a, L, b, c)$ when $[C R P]=0.00385 \mathrm{mg} / L$ and $J_{v}$ as the model output

\begin{tabular}{|l|l|}
\hline \multicolumn{2}{|c|}{$\boldsymbol{C R}_{\text {low }}: \boldsymbol{J}_{\boldsymbol{v}}$} \\
\hline starting values $(a, L, c)$ & estimated values \\
\hline \hline$(-10,22,0.03)$ & $(-10.555,23.863,0.027943)$ \\
$(-8,25,0.015)$ & $(-4.0636,12.342,0.027943)$ \\
$(-11.5,19,0.01)$ & $(-13.826,24.199,0.0006396)$ \\
$(-11.8,27,0.04)$ & $(-13.394,28.902,0.027943)$ \\
$(-7,18,0.01)$ & $(1.7336,2.0522,0.027943)$ \\
\hline
\end{tabular}

Table 3.23: Estimates for $(a, L, c)$ when $[C R P]=0.00385 \mathrm{mg} / L$ and $J_{v}$ as the model output

Similarly, when we use $J_{v}$ as the model output instead of $H c t$ and calculate estimates for $(a, L, b, c)$, the resulting estimates still lie in a wide range of values. We see this in 


\begin{tabular}{|l|l|}
\hline \multicolumn{2}{|c|}{$\boldsymbol{C R P}_{\text {low }}: \boldsymbol{J}_{\boldsymbol{v}}$} \\
\hline starting values $(L, b)$ & estimated values \\
\hline \hline$(22,0.91)$ & $(22.346,0.90598)$ \\
$(25,0.88)$ & $(22.346,0.90598)$ \\
$(19,0.9)$ & $(22.346,0.90598)$ \\
$(27,0.89)$ & $(22.346,0.90598)$ \\
$(18,0.78)$ & $(22.346,0.90598)$ \\
\hline
\end{tabular}

Table 3.24: Estimates for $(L, b)$ when $[C R P]=0.00385 \mathrm{mg} / L$ and $J_{v}$ as the model output

table 3.22. Also, there is an improvement in results when the three-parameter subset $(a, L, c)$ is estimated. From table 3.23 , we observe that $c$ can be estimated for all cases except the third one and its value converges to 0.027943 . Note that the nominal value for $c$ is 0.0278 . However, the other two parameters cannot be reliably estimated. Just like in the $H c t$ case, the parameter subset $(L, b)$ is identifiable when measurements for $J_{v}$ are used to calculate the estimates. We obtain estimated values of 22.346 for $L$ and 0.90598 for $b$, which are closer to their nominal values compared to the estimates for the Hct case.

\subsubsection{High CRP concentration}

Next, we consider the case $C R P_{h i g h}$, that is, $[C R P]=0.018 \mathrm{mg} / \mathrm{mL}$ and examine if we can get reliable estimates from $H c t$ and $J_{v}$ measurements. Tables 3.25, 3.26, and 3.27 present the results for the cases where Hct is used as the model output. We did estimation from $H c t$ measurements for the the same parameter subsets $(a, L, b, c)$, $(L, b, c)$, and $(L, b)$.

The estimates for the subset $(a, L, b, c)$ again lie in a wide range of values as we can see in table 3.25 and not one can be estimated with sufficient accuracy. For the subset $(L, b, c)$, we observe that the estimates for $L$ are around 22 for the first, third, and fifth entries in table 3.26 , and 15.145 for the second and fourth entries. This shows that for $C R P_{h i g h}, L$ may go to two minimum values, depending on the starting values. In table 3.27, the estimates for $(L, b)$ are not as definite as in $C R P_{\text {low }}$ since the fourth entry in the table has different values that the rest. However, the obtained estimates $(22.35,0.9057)$ are more accurate than the results obtained from $C R P_{\text {low }}$ and $H c t$ as the model output, because they are closer to the nominal parameter values of $L$ and $b$. 


\begin{tabular}{|l|l|}
\hline \multicolumn{2}{|c|}{$\boldsymbol{C R P}_{\text {high }}: \boldsymbol{H} \boldsymbol{c t}$} \\
\hline starting values $(a, L, b, c)$ & estimated values \\
\hline \hline$(-10,22,0.91,0.03)$ & $(-10.255,23.074,0.8678,0.0293)$ \\
$(-11.5,19,0.9,0.01)$ & $(-14.561,21.482,0.51735,0.014396)$ \\
$(-11.8,27,0.89,0.04)$ & $(-12.796,26.386,0.70402,0.044003)$ \\
$(-7,18,0.78,0.01)$ & $(-6.9794,18.789,0.77715,0.009838)$ \\
$(-8,25,0.88,0.02)$ & $(-6.9209,18.727,1.0197,0.024545)$ \\
\hline
\end{tabular}

Table 3.25: Estimates for $(a, L, b, c)$ when $[C R P]=0.018 \mathrm{mg} / L$ and $H c t$ as the model output

\begin{tabular}{|l|l|}
\hline \multicolumn{2}{|c|}{$\boldsymbol{C R P}_{\text {high }}:$ Hct } \\
\hline starting values $(L, b, c)$ & estimated values \\
\hline \hline$(22,0.91,0.03)$ & $(22.7791,0.8167,0.0325)$ \\
$(19,0.9,0.01)$ & $(15.145,0.86702,0.012482)$ \\
$(27,0.89,0.04)$ & $(22.35,0.72705,0.040297)$ \\
$(18,0.78,0.01)$ & $(15.145,0.69967,0.01318)$ \\
$(25,0.88,0.02)$ & $(22.35,0.90526,0.027816)$ \\
\hline
\end{tabular}

Table 3.26: Estimates for $(L, b, c)$ when $[C R P]=0.018 \mathrm{mg} / L$ and $H c t$ as the model output

\begin{tabular}{|l|l|}
\hline \multicolumn{2}{|c|}{$\boldsymbol{C R P}_{\text {high }}: \boldsymbol{H} \boldsymbol{H} \boldsymbol{t}$} \\
\hline starting values $(L, b)$ & estimated values \\
\hline \hline$(22,0.91)$ & $(22.35,0.90569)$ \\
$(25,0.88)$ & $(22.35,0.9057)$ \\
$(19,0.9)$ & $(22.35,0.9057)$ \\
$(27,0.89)$ & $(22.48,0.90882)$ \\
$(18,0.78)$ & $(22.35,0.9057)$ \\
\hline
\end{tabular}

Table 3.27: Estimates for $(L, b)$ when $[C R P]=0.018 \mathrm{mg} / L$ and $H c t$ as the model output

For the case $C R P_{h i g h}$ and $J_{v}$ is the model output, we look at estimates for the parameter subsets $(a, d, b, c),(L, b, c),(a, b, c),(d, b, c)$ and $(L, b)$. As we can see in table 3.28 , the subset of length four $(a, d, b, c)$ still does not provide accurate estimates for any of the parameters in the subset. For parameter vectors of length 3 , we consider three subsets: $(L, b, c),(a, b, c)$, and $(d, b, c)$. Each is a combination of a parameter from the function $L_{p}([C R P])$ and the parameters $b$ and $c$ from the function $\sigma([C R P])$. Tables 3.29 , 3.30, and 3.31 show that in all subsets, only one parameter out of the three can be estimated with sufficient accuracy. The parameter $L$ converges to 22.349 , a converges 


\begin{tabular}{|l|l|}
\hline \multicolumn{2}{|c|}{$\boldsymbol{C R P}_{\text {high }}: \boldsymbol{J}_{\boldsymbol{v}}$} \\
\hline starting values $(a, d, b, c)$ & estimated values \\
\hline \hline$(-10,60,0.91,0.03)$ & $(-9.6298,65.244,0.90454,0.027847)$ \\
$(-11.5,62,0.9,0.01)$ & $(-3.9827,2.5054,0.62025,0.089433)$ \\
$(-11.8,71,0.89,0.04)$ & $(-10.779,82.519,0.73283,0.039534)$ \\
$(-7,80,0.78,0.01)$ & $(-7.4639,38.598,3.108,0.014004)$ \\
$(-8,73,0.88,0.02)$ & $(-10.138,72.502,1.1319,0.022464)$ \\
\hline
\end{tabular}

Table 3.28: Estimates for $(a, d, b, c)$ when $[C R P]=0.018 \mathrm{mg} / L$ and $J_{v}$ as the model output

\begin{tabular}{|l|l|}
\hline \multicolumn{2}{|c|}{$\boldsymbol{C R P}_{\boldsymbol{h i g h}}: \boldsymbol{J}_{\boldsymbol{v}}$} \\
\hline starting values $(L, b, c)$ & estimated values \\
\hline \hline$(22,0.91,0.03)$ & $(22.349,0.81937,0.031873)$ \\
$(19,0.9,0.01)$ & $(22.349,1.6894,0.017629)$ \\
$(27,0.89,0.04)$ & $(22.349,0.71984,0.041358)$ \\
$(18,0.78,0.01)$ & $(22.349,0.6006,0.19742)$ \\
$(25,0.88,0.02)$ & $(22.349,0.91879,0.027341)$ \\
\hline
\end{tabular}

Table 3.29: Estimates for $(L, b, c)$ when $[C R P]=0.018 \mathrm{mg} / L$ and $J_{v}$ as the model output

\begin{tabular}{|l|l|}
\hline \multicolumn{2}{|c|}{$\boldsymbol{C R P}_{\text {high }}: \boldsymbol{J}_{\boldsymbol{v}}$} \\
\hline starting values $(a, b, c)$ & estimated values \\
\hline \hline$(-10,0.91,0.03)$ & $(-9.701,0.77839,0.034796)$ \\
$(-11.5,0.9,0.01)$ & $(-9.701,5.9354,0.011871)$ \\
$(-11.8,0.89,0.04)$ & $(-9.701,0.68603,0.047884)$ \\
$(-7,0.78,0.01)$ & $(-9.701,1.1174,0.022693)$ \\
$(-8,0.88,0.02)$ & $(-9.701,1.0802,0.02333)$ \\
\hline
\end{tabular}

Table 3.30: Estimates for $(a, b, c)$ when $[C R P]=0.018 \mathrm{mg} / L$ and $J_{v}$ as the model output

to -9.701 , and $d$ converges to 66.2162 . The values are all close to the nominal values $L_{0}=22.35, a_{0}=-9.70$, and $d_{0}=66.23$. Finally, table 3.32 shows the estimates when $L$ and $b$ are the only parameters to be estimated. This subset continues to give accurate and reliable estimates, this time with estimated values for $L=22.349$ and $b=0.90583$. 


\begin{tabular}{|l|l|}
\hline \multicolumn{2}{|c|}{$\boldsymbol{C R P}_{\boldsymbol{h i g h}}: \boldsymbol{J}_{\boldsymbol{v}}$} \\
\hline starting values $(d, b, c)$ & estimated values \\
\hline \hline$(60,0.91,0.03)$ & $(66.2162,0.8983,0.0281)$ \\
$(62,0.9,0.01)$ & $(66.2162,0.6632,0.0549)$ \\
$(71,0.89,0.04)$ & $(66.2162,0.7029,0.0442)$ \\
$(80,0.78,0.01)$ & $(66.2162,1.7428,0.0174)$ \\
$(73,0.88,0.02)$ & $(66.2162,0.9924,0.0252)$ \\
\hline
\end{tabular}

Table 3.31: Estimates for $(d, b, c)$ when $[C R P]=0.018 \mathrm{mg} / L$ and $J_{v}$ as the model output

\begin{tabular}{|l|l|}
\hline \multicolumn{2}{|c|}{$\boldsymbol{C R P}_{\boldsymbol{h i g h}}: \boldsymbol{J}_{\boldsymbol{v}}$} \\
\hline starting values $(L, b)$ & estimated values \\
\hline \hline$(22,0.91)$ & $(22.349,0.90583)$ \\
$(25,0.88)$ & $(22.349,0.90583)$ \\
$(19,0.9)$ & $(22.349,0.90583)$ \\
$(27,0.89)$ & $(22.349,0.90583)$ \\
$(18,0.78)$ & $(22.349,0.90583)$ \\
\hline
\end{tabular}

Table 3.32: Estimates for $(L, b)$ when $[C R P]=0.018 \mathrm{mg} / L$ and $J_{v}$ as the model output

\subsection{Parameter estimation with two model outputs}

Following the discussion in [89], we now do parameter estimation using simultaneously the two model outputs, hematocrit and fluid flux. We hope that using measurements for fluid flux measurements, in addition to the measurements for hematocrit, may improve the calculated parameter estimates.

In this section, we assume that the model output $f$ is given by

$$
f(t, \theta)=\left(f_{1}(t, \theta), f_{2}(t, \theta)\right)^{T}, \quad 0 \leq t \leq 60, \theta \in \mathcal{A},
$$

where $\theta=(a L d b c)^{T}$ belongs to an admissible set of parameters $\mathcal{A} \subset \mathbb{R}^{5}, f_{1}(\cdot)=H c t(\cdot)$, $f_{2}(\cdot)=J_{v}(\cdot)$, and $f_{1}, f_{2}$ are sufficiently smooth. We assume the simple case where the sampling times for the two outputs are the same and are given by $0 \leq t_{1}<t_{2}<\ldots<$ $t_{N} \leq 60$. Furthermore, we assume that the measurements are available at each $t_{j}$, and that there exists a nominal parameter $\theta_{0}$ such that each measurement can be expressed as

$$
y_{i}\left(t_{j}, \theta_{0}\right)=f_{i}\left(t_{j}, \theta_{0}\right)+\epsilon_{i}\left(t_{j}\right), \quad i=1,2, j=1, \ldots, N,
$$

where $\epsilon_{i}\left(t_{j}\right)$ is the measurement error at time $t_{j}$. As before, $\epsilon_{i}(t)$ is a realization of a random variable $\mathcal{E}_{i}(t)$, representing a noise process for $t \in[0,60], i=1,2$. We have the following assumptions on the random variables $\mathcal{E}_{i}\left(t_{j}\right), i=1,2, j=1, \ldots, N$ : 
1. $\mathcal{E}_{i}(\cdot)$ does not depend on $\theta$,

2. $\mathcal{E}_{1}\left(t_{j}\right)$ and $\mathcal{E}_{2}\left(t_{j}\right), j=1, \ldots, N$ are independent and identically distributed,

3. $\mathbb{E}\left(\mathcal{E}_{i}(t)\right)=0$ for $i=1,2,0 \leq t \leq 60$, and

4. $\operatorname{Var}\left(\mathcal{E}_{i}(t)\right)=\sigma_{i, 0}^{2}$, finite and constant, for $i=1,2,0 \leq t \leq 60$.

For $i=1,2$ and $\theta_{0}, \theta \in \mathcal{A}$, denote by $Y_{i}(\theta)=\left(y_{i}\left(t_{1}, \theta\right), \ldots, y_{i}\left(t_{N}, \theta\right)\right)^{T}$ and $F_{i}(\theta)=$ $\left(f_{i}\left(t_{1}, \theta\right), \ldots, f_{i}\left(t_{N}, \theta\right)\right)^{T}$. To calculate for the parameter estimates, we choose a similar error functional $J$ of least squares form given by

$$
J\left(\theta_{0}, \theta\right)=J_{1}\left(\theta_{0}, \theta\right)+J_{2}\left(\theta_{0}, \theta\right)
$$

where for $i=1,2$

$$
\begin{aligned}
J_{i}\left(\theta_{0}, \theta\right) & =\frac{1}{\sigma_{i, 0}^{2}} \sum_{j=1}^{N}\left(y_{i}\left(t_{j}, \theta_{0}\right)-f_{i}\left(t_{j}, \theta\right)\right)^{2} \\
& =\frac{1}{\sigma_{i, 0}^{2}}\left\|Y_{i}\left(\theta_{0}\right)-F_{i}(\theta)\right\|^{2}
\end{aligned}
$$

Finally, minimizing the functional $J$ in $(3.29)$ over all $\theta \in \mathcal{A}$ gives the estimate $\hat{\theta}$; that is,

$$
\hat{\theta}\left(\theta_{0}\right)=\underset{\theta \in \mathcal{A}}{\operatorname{argmin}} J\left(\theta_{0}, \theta\right)
$$

Like the measurement errors $\epsilon_{i}\left(t_{j}\right)$, the measurements $y_{i}\left(t_{j}, \theta_{0}\right)$ in 3.28 are realizations of a random variable $\mathcal{Y}_{i}\left(t, \theta_{0}\right), t \in[0,60]$ and that the following equation is satisfied:

$$
\mathcal{Y}_{i}\left(t_{j}, \theta_{0}\right)=f_{i}\left(t_{j}, \theta_{0}\right)+\mathcal{E}_{i}\left(t_{j}\right), \quad i=1,2, j=1, \ldots, N \text {. }
$$

It follows from the assumptions that the expected value and variance of the measurement process $\mathcal{Y}_{i}\left(t, \theta_{0}\right)$ are $\mathbb{E}\left(\mathcal{Y}_{i}\left(t, \theta_{0}\right)\right)=f_{i}\left(t, \theta_{0}\right)$ and $\operatorname{Var}\left(\mathcal{Y}_{i}\left(t, \theta_{0}\right)\right)=\sigma_{i, 0}^{2}$, respectively. The estimate $\hat{\theta}\left(\theta_{0}\right)$ in 3.30 is also a realization of a random variable $\hat{\Theta}\left(\theta_{0}\right)$, called the least-squares estimator for this parameter identification problem.

In the following tables, we present the estimates for the parameter vector $(a, L, d, b, c)$ from generated measurements of the two model outputs $H c t(t)$ and $J_{v}(t)$. Table 3.33 shows the estimates when $[C R P]=0.00385 \mathrm{mg} / \mathrm{mL}$, while table 3.34 shows the estimates when $[C R P]=0.018 \mathrm{mg} / \mathrm{mL}$. The nominal values for the parameters are the

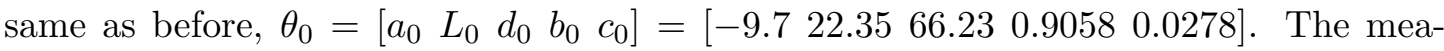
surements taken from the subinterval $[10,50]$ are used in the estimation of parameters. 


\begin{tabular}{|l|l|}
\hline \multicolumn{2}{|c|}{$\boldsymbol{C R} \boldsymbol{P P}_{\text {low }}: \boldsymbol{H} \boldsymbol{c t}$ and $\boldsymbol{J}_{\boldsymbol{v}}$} \\
\hline starting values $(a, L, d, b, c)$ & estimated values \\
\hline \hline$(-10,22,60,0.91,0.03)$ & $(-9.8881,22.878,61.23,0.9027,0.030692)$ \\
$(-8,25,73,0.88,0.015)$ & $(-8.75,20.43,73.102,0.94673,0.015294)$ \\
$(-11.5,19,62,0.9,0.01)$ & $(-8.2585,19.742,67.784,1.0278,0.010092)$ \\
$(-11.8,27,71,0.89,0.04)$ & $(-12.128,26.459,70.778,0.89655,0.040799)$ \\
$(-7,18,80,0.78,0.01)$ & $(-6.5485,16.607,71.665,0.99181,0.011615)$ \\
\hline
\end{tabular}

Table 3.33: Estimates for $(a, L, d, b, c)$ when $[C R P]=0.00385 \mathrm{mg} / \mathrm{mL}$ using two model outputs

\begin{tabular}{|l|l|}
\hline \multicolumn{2}{|c|}{$\boldsymbol{C R} \boldsymbol{P P}_{\text {high }}:$ Hct and $\boldsymbol{J}_{\boldsymbol{v}}$} \\
\hline starting values $(a, L, d, b, c)$ & estimated values \\
\hline \hline$(-10,22,60,0.91,0.03)$ & $(-9.972,23.085,62.38,0.86291,0.029564)$ \\
$(-11.5,19,62,0.9,0.01)$ & $(4.5133,37.585,-137.2,0.66691,0.053534)$ \\
$(-11.8,27,71,0.89,0.04)$ & $(-13.657,26.596,74.775,0.71969,0.04138)$ \\
$(-7,18,80,0.78,0.01)$ & $(-6.8981,19.26,81.105,0.77671,0.009728)$ \\
$(-8,25,73,0.88,0.02)$ & $(-8.1758,19.975,70.979,1.0305,0.024311)$ \\
\hline
\end{tabular}

Table 3.34: Estimates for $(a, L, d, b, c)$ when $[C R P]=0.018 \mathrm{mg} / \mathrm{mL}$ using two model outputs

We see from the tables that there are not much improvement in the estimation of the five parameters even if measurements from two model outputs are used. The parameters $a, L, d, b$, and $c$ cannot be reliably estimated in both cases $C R P_{\text {low }}$ and $C R P_{\text {high }}$. Moreover, we also observe that it is more probable in the $C R P_{\text {high }}$ case to obtain estimates which are farther from the nominal values. For instance, if we start the search at $(-11.5,19,62,0.9,0.01)$, the estimated values for $C R P_{h i g h}$ in table 3.34 are $(4.5133,37.585,-137.2,0.66691,0.053534)$. These are farther from the nominal values than if we consider the estimated values for $C R P_{\text {low }}$ in table 3.33 , which are $(-8.2585,19.742,67.784,1.0278,0.010092)$

Next, we look at estimates for parameter subsets of length 4 and 3 . We present the results in the following subsections.

\subsubsection{Estimates for parameter subsets of length 4 using 2 outputs}

Tables 3.35 and 3.36 show the parameter estimates for the subset $(a, L, b, c)$ for the two cases $C R P_{\text {low }}$ and $C R P_{\text {high }}$. Again, we observe that this subset cannot be reliably estimated given measurements from the two model outputs. Also, it is more likely that better estimates can be obtained for the case $C R P_{\text {low }}$ than $C R P_{\text {high }}$ because the 
estimates in the latter case tend to be farther from the nominal values.

\begin{tabular}{|l|l|}
\hline \multicolumn{2}{|c|}{$\boldsymbol{C R P}_{\text {low }}: \boldsymbol{H} \boldsymbol{c t}$ and $\boldsymbol{J}_{\boldsymbol{v}}$} \\
\hline starting values $(a, L, b, c)$ & estimated values \\
\hline \hline$(-10,22,0.91,0.03)$ & $(-9.8208,22.5663,0.9024,0.0310)$ \\
$(-8,25,0.88,0.015)$ & $(-8.6313,20.4551,0.9424,0.0159)$ \\
$(-11.5,19,0.9,0.01)$ & $(-8.5481,20.3075,1.0232,0.0103)$ \\
$(-11.8,27,0.89,0.04)$ & $(-12.0367,26.4995,0.8965,0.0409)$ \\
$(-7,18,0.78,0.01)$ & $(-6.0852,15.9359,1.0524,0.0094)$ \\
\hline
\end{tabular}

Table 3.35: Estimates for $(a, L, b, c)$ when $[C R P]=0.00385 \mathrm{mg} / \mathrm{mL}$ using two model outputs

\begin{tabular}{|l|l|}
\hline \multicolumn{2}{|c|}{$\boldsymbol{C R P}_{\text {high }}: \boldsymbol{H} \boldsymbol{c t}$ and $\boldsymbol{J}_{\boldsymbol{v}}$} \\
\hline starting values $(a, L, b, c)$ & estimated values \\
\hline \hline$(-10,22,0.91,0.03)$ & $(-10.236,23.049,0.86272,0.029573)$ \\
$(-11.5,19,0.9,0.01)$ & $(-341.52,454.9,0.60001,0.21001)$ \\
$(-11.8,27,0.89,0.04)$ & $(-13.931,27.866,0.71988,0.041351)$ \\
$(-7.5,18,0.78,0.01)$ & $(-0.94174,10.933,0.79321,0.033629)$ \\
$(-8,25,0.88,0.02)$ & $(-7.1474,19.023,1.0098,0.024775)$ \\
\hline
\end{tabular}

Table 3.36: Estimates for $(a, L, b, c)$ when $[C R P]=0.018 \mathrm{mg} / \mathrm{mL}$ using two model outputs

We have also included results when the parameter subset $(a, d, b, c)$ is estimated. This subset has the best selection score $\alpha$ for the case $C R P_{h i g h}$ when $J_{v}$ is used as the model output. We see from table 3.37 that none of the parameters can be estimated reliably when two model outputs are used in the estimation.

\begin{tabular}{|l|l|}
\hline \multicolumn{2}{|c|}{$\boldsymbol{C R P}_{\boldsymbol{h i g h}}: \boldsymbol{H} \boldsymbol{c t}$ and $\boldsymbol{J}_{\boldsymbol{v}}$} \\
\hline starting values $(a, d, b, c)$ & estimated values \\
\hline \hline$(-10,60,0.91,0.03)$ & $(-9.6176,65.096,0.86291,0.029563)$ \\
$(-11.5,62,0.9,0.01)$ & $(-14.857,341.34,4.0619,0.012991)$ \\
$(-11.8,71,0.89,0.04)$ & $(-10.595,79.552,0.70495,0.043847)$ \\
$(-7,80,0.78,0.01)$ & $(-7.3318,80.006,0.78204,0.010011)$ \\
$(-8,73,0.88,0.02)$ & $(-9.4648,63.012,1.0524,0.023858)$ \\
\hline
\end{tabular}

Table 3.37: Estimates for $(a, d, b, c)$ when $[C R P]=0.018 \mathrm{mg} / \mathrm{mL}$ using two model outputs 
In addition, we consider the subsets $(a, L, d, b)$ and $(a, L, d, c)$, where only one parameter from the function for $\sigma$ appears. The parameters $a, L$, and $d$ appear in the function $L_{p}([C R P])$, while $b$ and $c$ appear in the function $\sigma([C R P])$. We see in tables 3.38 and 3.39 that $b$ converges to the value 0.9058 , while the rest of the parameters range in some large neighborhood of their respective nominal values. In tables 3.40 and 3.41, $c$ converges to 0.0278 while the rest do not converge. However, there is one entry in table 3.41 for the case $C R P_{\text {high }}$ that the parameter $c$ did not converge to 0.0278. From these results, we observe that it is possible to obtain good estimates for either $b$ or $c$ when only one of them appears in a subset rather than when they both appear in a subset.

\begin{tabular}{|l|l|}
\hline \multicolumn{2}{|c|}{$\boldsymbol{C R P}_{\text {low }}: \boldsymbol{H} \boldsymbol{c t}$ and $\boldsymbol{J}_{\boldsymbol{v}}$} \\
\hline starting values $(a, L, d, b)$ & estimated values \\
\hline \hline$(-10,22,60,0.91)$ & $(-9.9335,22.9802,60.7049,0.9058)$ \\
$(-8,25,73,0.88)$ & $(-8.8952,20.6580,73.8883,0.9058)$ \\
$(-11.5,19,62,0.9)$ & $(-9.7482,22.6578,60.4569,0.9058)$ \\
$(-11.8,27,71,0.89)$ & $(-11.9844,26.1900,71.1561,0.9058)$ \\
$(-7,18,80,0.78)$ & $(-7.1125,17.4857,75.5608,0.9058)$ \\
\hline
\end{tabular}

Table 3.38: Estimates for $(a, L, d, b)$ when $[C R P]=0.00385 \mathrm{mg} / \mathrm{mL}$ using two model outputs

\begin{tabular}{|l|l|}
\hline \multicolumn{2}{|c|}{$\boldsymbol{C R P}_{\boldsymbol{h i g h}} \boldsymbol{:} \boldsymbol{H} \boldsymbol{c t}$ and $\boldsymbol{J}_{\boldsymbol{v}}$} \\
\hline starting values $(a, L, d, b)$ & estimated values \\
\hline \hline$(-10,22,60,0.91)$ & $(-9.7921,23.042,60.469,0.90582)$ \\
$(-8,25,73,0.88)$ & $(-8.8382,20.394,76.481,0.90582)$ \\
$(-11.5,19,62,0.9)$ & $(-9.1978,21.641,66.831,0.90582)$ \\
$(-11.8,27,71,0.89)$ & $(-12.594,25.527,71.962,0.90582)$ \\
$(-7,18,80,0.78)$ & $(-6.8691,17.781,78.809,0.90582)$ \\
\hline
\end{tabular}

Table 3.39: Estimates for $(a, L, d, b)$ when $[C R P]=0.018 \mathrm{mg} / \mathrm{mL}$ using two model outputs

\subsubsection{Estimates for parameter subsets of length 3 using 2 outputs}

In this subsection, we present estimates for the subsets $(L, b, c)$ and $(a, L, c)$ for the cases $C R P_{\text {low }}$ and $C R P_{\text {high }}$ using measurements from two model outputs. Tables 3.42 and 3.43 are the estimates for $(L, b, c)$, while tables 3.44 and 3.45 are the estimates for $(a, L, c)$. 


\begin{tabular}{|l|l|}
\hline \multicolumn{2}{|c|}{$\boldsymbol{C R P}_{\text {low }}:$ Hct and $\boldsymbol{J}_{\boldsymbol{v}}$} \\
\hline starting values $(a, L, d, c)$ & estimated values \\
\hline \hline$(-10,22,60,0.03)$ & $(-10.7,24.608,54.628,0.027808)$ \\
$(-8,25,73,0.015)$ & $(-7.172,17.768,69.47,0.027808)$ \\
$(-11.5,19,62,0.01)$ & $(-2.6508,9.8587,65.109,0.027808)$ \\
$(-11.8,27,71,0.04)$ & $(-14.821,31.546,64.251,0.027808)$ \\
$(-7,18,80,0.01)$ & $(-4.4948,14.08,25.69,0.027808)$ \\
\hline
\end{tabular}

Table 3.40: Estimates for $(a, L, d, c)$ when $[C R P]=0.00385 \mathrm{mg} / \mathrm{mL}$ using two model outputs

\begin{tabular}{|l|l|}
\hline \multicolumn{2}{|c|}{$\boldsymbol{C R P}_{\text {high }}: \boldsymbol{H} \boldsymbol{\text { ct }}$ and $\boldsymbol{J}_{\boldsymbol{v}}$} \\
\hline starting values $(a, L, d, c)$ & estimated values \\
\hline \hline$(-10,22,60,0.03)$ & $(-10.087,23.313,61.645,0.027801)$ \\
$(-11.5,19,62,0.01)$ & $(-12.927,24.104,94.302,0.027801)$ \\
$(-11.8,27,71,0.04)$ & $(-13.062,25.522,78.254,0.027801)$ \\
$(-7,18,80,0.01)$ & $(-7.0314,19.06,79.875,0.010229)$ \\
$(-8,25,73,0.02)$ & $(-8.1451,20.524,63.926,0.027801)$ \\
\hline
\end{tabular}

Table 3.41: Estimates for $(a, L, d, c)$ when $[C R P]=0.018 \mathrm{mg} / \mathrm{mL}$ using two model outputs

\begin{tabular}{|l|l|}
\hline \multicolumn{2}{|c|}{$\boldsymbol{C R P}_{\text {low }}: \boldsymbol{H} \boldsymbol{c t}$ and $\boldsymbol{J}_{\boldsymbol{v}}$} \\
\hline starting values $(L, b, c)$ & estimated values \\
\hline \hline$(22,0.91,0.03)$ & $(22.3520,0.9031,0.0302)$ \\
$(25,0.88,0.015)$ & $(22.3520,0.9420,0.0159)$ \\
$(19,0.9,0.01)$ & $(22.3520,1.0475,0.0095)$ \\
$(27,0.89,0.04)$ & $(22.3520,0.8958,0.0427)$ \\
$(18,0.78,0.015)$ & $(22.3520,0.9589,0.0140)$ \\
\hline
\end{tabular}

Table 3.42: Estimates for $(L, b, c)$ when $[C R P]=0.00385 \mathrm{mg} / \mathrm{mL}$ using two model outputs

We see from the tables that one parameter among the three can be estimated from measurements of $H c t$ and $J_{v}$ on the interval $[10,50]$. In the subset $(L, b, c)$, the parameter $L$ can be estimated in both cases. The obtained estimate for $L=22.3505$ in $C R P_{\text {high }}$ is better than the estimate obtained in $C R P_{\text {low }}$, which is $L=22.3520$. But it is more possible for $L$ to converge to another value when $C R P$ is high. For the subset $(a, L, c)$, the parameter $c$ can be estimated and the calculated estimate for $c$ in both cases is equal to 0.0278. We observe again that if both $b$ and $c$ appear in a subset of length 3 , then neither of these two parameters can be estimated with sufficient accuracy. On the 


\begin{tabular}{|l|l|}
\hline \multicolumn{2}{|c|}{$\boldsymbol{R R P}_{\boldsymbol{h i g h}}: \boldsymbol{H} \boldsymbol{c t}$ and $\boldsymbol{J}_{\boldsymbol{v}}$} \\
\hline starting values $(L, b, c)$ & estimated values \\
\hline \hline$(22,0.91,0.03)$ & $(22.3505,0.7825,0.0345)$ \\
$(19,0.9,0.01)$ & $(22.3505,1.5894,0.0182)$ \\
$(27,0.89,0.04)$ & $(22.3505,0.7038,0.0441)$ \\
$(18,0.78,0.015)$ & $(14.2433,0.5368,0.0136)$ \\
$(25,0.88,0.02)$ & $(22.3505,0.9517,0.0263)$ \\
\hline
\end{tabular}

Table 3.43: Estimates for $(L, b, c)$ when $[C R P]=0.018 \mathrm{mg} / \mathrm{mL}$ using two model outputs

\begin{tabular}{|l|l|}
\hline \multicolumn{2}{|c|}{$\boldsymbol{C R P}_{\text {low }}: \boldsymbol{H} \boldsymbol{c t}$ and $\boldsymbol{J}_{\boldsymbol{v}}$} \\
\hline starting values $(a, L, c)$ & estimated values \\
\hline \hline$(-10,22,0.03)$ & $(-10.6804,24.0922,0.0278)$ \\
$(-8,25,0.015)$ & $(-3.8777,12.0178,0.0278)$ \\
$(-11.5,19,0.01)$ & $(5.7912,-5.1439,0.0278)$ \\
$(-11.8,27,0.04)$ & $(-16.2120,33.9103,0.0278)$ \\
$(-7,18,0.01)$ & $(0.9479,3.4527,0.0278)$ \\
\hline
\end{tabular}

Table 3.44: Estimates for $(a, L, c)$ when $[C R P]=0.00385 \mathrm{mg} / \mathrm{mL}$ using two model outputs

\begin{tabular}{|l|l|}
\hline \multicolumn{2}{|c|}{$\boldsymbol{C R P}_{\boldsymbol{h i g h}} \boldsymbol{:} \boldsymbol{H c t}$ and $\boldsymbol{J}_{\boldsymbol{v}}$} \\
\hline starting values $(a, L, c)$ & estimated values \\
\hline \hline$(-10,22,0.03)$ & $(-10.2531,23.0716,0.0278)$ \\
$(-11.5,19,0.01)$ & $(-3.2553,13.9493,0.0278)$ \\
$(-11.8,27,0.04)$ & $(-13.5654,27.3893,0.0278)$ \\
$(-7,18,0.01)$ & $(-1.8662,12.1385,0.0278)$ \\
$(-8,25,0.02)$ & $(-8.2129,20.4119,0.0278)$ \\
\hline
\end{tabular}

Table 3.45: Estimates for $(a, L, c)$ when $[C R P]=0.018 \mathrm{mg} / \mathrm{mL}$ using two model outputs

other hand, if only one of these two parameters appear in a subset of length 3 , then that parameter is the one that can be estimated accurately.

\subsubsection{Global search method}

As we have seen from the results in the previous sections, it is difficult to obtain reliable estimates for parameter subsets of lengths 3,4 , and 5 . It is possible that the cost functional has a number of local minima. We now use genetic algorithm (GA) command in Matlab and fminsearch to obtain estimates for different parameter subsets subject to some bound. In implementing the GA, we set the bounds for each parameter at $\pm 15 \%$ 
and $\pm 30 \%$ of the nominal values, except for the upper bound for $b$ which is fixed at 1. In table 3.46, the bound is set to $15 \%$ from the nominal parameter value. That is, $a, L, d, b$ and $c$ lie between the values $-9.70 \cdot(1 \pm 0.15), 22.35 \cdot(1 \pm 0.15), 66.23 \cdot(1 \pm 0.15)$, $0.9058 \cdot 0.85$ and 1 , and $0.0278 \cdot(1 \pm 0.15)$, respectively. Table 3.47 shows the results when the bound is set to $30 \%$. Estimates are calculated from the generated measurements for the two model outputs $H c t$ and $J_{v}$. We present the results for the case $C R P_{\text {low }}$ in the following tables.

\begin{tabular}{|l|l|l|l|l|l|}
\hline \multicolumn{7}{|c|}{$\boldsymbol{C R P}_{\text {low }}:$ Hct and $\boldsymbol{J}_{\boldsymbol{v}}, \mathbf{1 5} \%$} \\
\hline parameter vector & $a$ & $L$ & $d$ & $b$ & $c$ \\
\hline \hline$(a, L, d, b, c)$ & -9.6778 & 22.1137 & 71.5878 & 0.9137 & 0.0231 \\
$(a, d, b, c)$ & -9.7235 & & 67.3912 & 0.9189 & 0.0210 \\
$(a, L, b, c)$ & -9.1520 & 21.3794 & & 0.9119 & 0.0239 \\
$(a, L, d, b)$ & -9.4771 & 21.9512 & 66.3726 & 0.9058 & \\
$(a, L, d, c)$ & -8.5200 & 20.9075 & 60.9075 & & 0.0278 \\
$(L, b, c)$ & & 22.3520 & & 0.9098 & 0.0251 \\
$(a, b, c)$ & -9.6989 & & & 0.9368 & 0.0168 \\
$(d, b, c)$ & & & 66.2827 & 0.9108 & 0.0245 \\
$(a, L, c)$ & -6.4914 & 16.6570 & & & 0.0278 \\
$(a, L, d)$ & -9.9416 & 22.4340 & 75.4535 & & \\
\hline
\end{tabular}

Table 3.46: Estimates for different parameter subsets using GA and fminsearch; $[C R P]=0.00385 \mathrm{mg} / \mathrm{mL}$ using two model outputs; bounds are $15 \%$ from the nominal values

We see from the tables that although not all the parameters in a subset are very close to their nominal values, there is still a significant improvement because the estimates lie closer to the nominal values compared to the values we have obtained before. The subsets $(a, L, d, b)$ and $(a, L, d, c)$ give very good estimates for $b$ and $c$ when CRP level is low. At least one of the parameters in the subsets of length 3 can be estimated accurately. The parameters $L, a$, and $d$ can be estimated reliably in the subsets $(L, b, c)$, $(a, b, c)$, and $(d, b, c)$, respectively. Meanwhile, $c$ can be identified in the subset $(a, L, c)$ but none can be identified in the subset $(a, L, d)$.

Tables 3.48 and 3.49 give the GA and fminsearch results for the case $C R P_{h i g h}$ when the bounds are set to $15 \%$ and $30 \%$, respectively. 


\begin{tabular}{|l|l|l|l|l|l|}
\hline \multicolumn{7}{|c|}{$\boldsymbol{R P}_{\text {low }}: \boldsymbol{H} \boldsymbol{c t}$ and $\boldsymbol{J}_{\boldsymbol{v}}, \mathbf{3 0} \%$} \\
\hline parameter vector & $a$ & $L$ & $d$ & $b$ & $c$ \\
\hline \hline$(a, L, d, b, c)$ & -7.6129 & 19.3159 & 45.7354 & 0.9207 & 0.0204 \\
$(a, d, b, c)$ & -9.2633 & & 45.7447 & 0.9660 & 0.0133 \\
$(a, L, b, c)$ & -8.0236 & 19.3764 & & 0.9245 & 0.0193 \\
$(a, L, d, b)$ & -7.6503 & 19.4193 & 44.7231 & 0.9058 & \\
$(a, L, d, c)$ & -10.4106 & 24.3666 & 47.9092 & & 0.0278 \\
$(L, b, c)$ & & 22.3520 & & 0.9198 & 0.0207 \\
$(a, b, c)$ & -9.6989 & & & 0.9425 & 0.0159 \\
$(d, b, c)$ & & & 66.2827 & 0.9221 & 0.0200 \\
$(a, L, c)$ & -9.2203 & 21.5005 & & & 0.0278 \\
$(a, L, d)$ & -7.3526 & 18.5928 & 53.2411 & & \\
\hline
\end{tabular}

Table 3.47: Estimates for different parameter subsets using GA and fminsearch; $[C R P]=0.00385 \mathrm{mg} / \mathrm{mL}$ using two model outputs; bounds are $30 \%$ from the nominal values

\begin{tabular}{|l|l|l|l|l|l|}
\hline \multicolumn{7}{|c|}{$\boldsymbol{C R P}_{\boldsymbol{h i g h}}: \boldsymbol{H} \boldsymbol{c t}$ and $\boldsymbol{J}_{\boldsymbol{v}}, \mathbf{1 5} \%$} \\
\hline parameter vector & $a$ & $L$ & $d$ & $b$ & $c$ \\
\hline \hline$(a, L, d, b, c)$ & -10.1093 & 22.0667 & 75.4806 & 0.8789 & 0.0289 \\
$(a, d, b, c)$ & -9.3115 & & 60.9626 & 0.8668 & 0.0294 \\
$(a, L, b, c)$ & -8.9920 & 21.4276 & & 0.8275 & 0.0314 \\
$(a, L, d, b)$ & -10.3073 & 23.2448 & 65.1817 & 0.9058 & \\
$(a, L, d, c)$ & -8.5312 & 21.0904 & 63.2914 & & 0.0278 \\
$(L, b, c)$ & & 22.3505 & & 0.8575 & 0.0298 \\
$(a, b, c)$ & -9.6996 & & & 0.8741 & 0.0291 \\
$(d, b, c)$ & & & 66.2355 & 0.8388 & 0.0308 \\
$(a, L, c)$ & -10.5991 & 23.5225 & & & 0.0278 \\
$(a, L, d)$ & -8.7809 & 21.8002 & 59.3629 & & \\
\hline
\end{tabular}

Table 3.48: Estimates for different parameter subsets using GA and fminsearch; $[C R P]=0.018 \mathrm{mg} / \mathrm{mL}$ using two model outputs; bounds are $15 \%$ from the nominal values

The same can be observed for the results of GA and fminsearch when CRP is high. Generally, the estimates are better than before since they lie close, but not equal, to the nominal parameter values. The subsets $(a, L, d, b)$ and $(a, L, d, c)$ give accurate estimates for $b$ and $c$. At least one among the three parameters in subsets of length 3 can be estimated with sufficient accuracy. The subset $(a, b, c)$ in table 3.49 is noteworthy for giving very good estimates for all the parameters: $(-9.6996,0.9088,0.0277)$. 


\begin{tabular}{|l|l|l|l|l|l|}
\hline \multicolumn{7}{|c|}{$\boldsymbol{R P}_{\boldsymbol{h i g h}}: \boldsymbol{H c t}$ and $\boldsymbol{J}_{\boldsymbol{v}}, \mathbf{3 0} \%$} \\
\hline parameter vector & $a$ & $L$ & $d$ & $b$ & $c$ \\
\hline \hline$(a, L, d, b, c)$ & -7.6265 & 20.7936 & 53.8126 & 0.8141 & 0.0322 \\
$(a, d, b, c)$ & -10.8817 & & 84.2473 & 0.9372 & 0.0267 \\
$(a, L, b, c)$ & -9.6718 & 22.3138 & & 0.8385 & 0.0308 \\
$(a, L, d, b)$ & -7.8079 & 19.4788 & 71.3182 & 0.9058 & \\
$(a, L, d, c)$ & -12.3999 & 26.0167 & 64.8946 & & 0.0278 \\
$(L, b, c)$ & & 22.3505 & & 0.7881 & 0.0340 \\
$(a, b, c)$ & -9.6996 & & & 0.9088 & 0.0277 \\
$(d, b, c)$ & & & 66.2355 & 1 & 0.0248 \\
$(a, L, c)$ & -8.8818 & 21.2839 & & & 0.0278 \\
$(a, L, d)$ & -9.2027 & 22.0543 & 62.4845 & & \\
\hline
\end{tabular}

Table 3.49: Estimates for different parameter subsets using GA and fminsearch; $[C R P]=0.018 \mathrm{mg} / \mathrm{mL}$ using two model outputs; bounds are $30 \%$ from the nominal values

We have also included in this subsection estimates for the subset $(a, L, d)$. Recall from the subset selection results that this subset has the worst selection score in two of the four cases: when $J_{v}$ is the model output for $C R P_{\text {low }}$ and when $H c t$ is the model output for $C R P_{\text {high }}$. Indeed, we do not obtain accurate estimates for any of the parameters in the subset $(a, L, d)$. From observation, we can say that a good combination of parameters of length 3 contains parameters that belong to the two functions. It must consist of $a, L$, or $d$ from $L_{p}([C R P])$ and $b$ or $c$ from $\sigma([C R P])$. Moreover, we expect that the single parameter belonging to the other function is the one that can be estimated with sufficient accuracy. For instance in $(L, b, c)$, we expect to obtain good estimates for $L$ since both $b$ and $c$ are parameters for $\sigma([C R P])$ while $L$ is for the other function. 


\section{Chapter 4}

\section{Conclusion}

In this work, we have presented a model which aims to incorporate inflammation to a model of vascular refilling [13] during ultrafiltration in a short-term hemodialysis. It is of interest to understand how vascular refilling during HD might be affected by the presence of inflammation. Inflammation is highly prevalent in patients undergoing HD 27 and is believed to greatly contribute in promoting cardiovascular disease progression 8. It is well-known that inflammation increases vascular permeability. In this study, we quantify the degree of inflammation based on the concentration of the inflammatory biomarker C-reactive protein (CRP) in the blood. The high CRP concentration among HD patients is linked to the presence of inflammation. We have explored how the properties of the capillary wall, namely filtration coefficient $L_{p}$ and reflection coefficient $\sigma$, are affected as the degree of inflammation based on the CRP level changes.

Functions relating CRP concentration to the coefficients $L_{p}$ and $\sigma$ were formulated and five additional parameters: $a, L, d, b$, and $c$ were introduced. Assuming that only these five parameters change as CRP concentration changes, sensitivity analysis, subset selection based on asymptotic standard errors, and parameter estimation are done. Estimates were obtained from generated measurements for two outputs, hematocrit (Hct) and fluid flux $\left(J_{v}\right)$. Considering $J_{v}$ as a model output, in addition to $H c t$, is an attempt to improve parameter estimates. However, we have seen that using measurements for $J_{v}$ do not improve the estimates significantly. It is worthwhile to consider other model outputs in doing parameter estimation in the future.

The results of the sensitivity analysis gave us an idea regarding the time period when the parameters provide the most information that is useful for the parameter estimation method. However, it did not provide information regarding the dependency among the parameters. Since none of the parameters in the subset $(a, L, d)$ is identifiable, then it may mean that $a, L$, and $d$ are somehow dependent. Moreover, the selection score for the subset $(a, L, d)$ is one of the largest based on asymptotic standard errors, which means that uncertainty is high for at least one of the parameters in this subset. The subset selection algorithm served as guide in determining which subset of parameters 
may be estimated accurately. Other basis for the selection score in determining best subset combination may be explored in further studies.

From the results of parameter estimation, it is probable that the problem has a number of local minima. A local search method is not always enough to obtain relatively good estimates, especially for parameter subsets containing 3 or more parameters. We have observed that combining GA with fminsearch improves significantly the obtained results.

When estimating all five parameters, not one can be estimated reliably for the cases $C R P_{\text {low }}$ and $C R P_{\text {high }}$. For the four- and three-parameter subsets, it is possible to get reliable estimates for at least one parameter. In the four-parameter subsets $(a, L, d, b)$ and $(a, L, d, c)$, the parameters $b$ and $c$ can be identified. On the other hand, if both $b$ and $c$ appear on the parameter subset, then neither can be reliably estimated. We conclude that when estimating a four-parameter subset, either $b$ or $c$ is identifiable provided that the subset consists of the three parameters $a, L$, and $d$ plus either $b$ or $c$. This is consistent with the sensitivity analysis result that $b$ and $c$ are the most sensitive parameters and hence, we expect to obtain good estimates for these parameters.

It is also possible to get sufficiently accurate estimates for $L, a$ or $c$ when estimating parameter subsets of length 3 using only a local search method. But when local and global search methods are used together, then at least one parameter can always be identified except for the subset $(a, L, d)$. The subset $(a, L, d)$ consists of parameters from the function $L_{p}([C R P])$ only. Besides $(a, L, d)$, all other subsets of length 3 consist of a combination of parameters from the functions $L_{p}([C R P])$ and $\sigma([C R P])$, where one parameter belongs to the other function than the remaining two parameters. The simulated results show that the single parameter belonging to the other function is the one that can be identified.

Finally, the two-parameter subset $(L, b)$ can always be identified from hematocrit and fluid flux measurements for both cases of CRP concentration.

The simulations also show that reliable estimates are more likely to be obtained for the case $C R P_{\text {low }}$ than for the case $C R P_{\text {high }}$. Compare for instance tables 3.20 and 3.26 . where the parameters $L, b$, and $c$ are estimated using hematocrit measurements. The parameter $L$ converges to 22.373 in all five starting values for $C R P_{\text {low }}$, while $L$ converges to $22.35,22.7791$ or 15.145 starting at the same values for $C R P_{h i g h}$.

Overall, the results of the parameter estimation are not excellent, despite considering parameter subsets or measurements for two model outputs. Only the subset $(L, b)$ gives sufficiently accurate estimates for all the parameters in the subset. Because of this, it would be necessary to reassess the model assumptions. Particularly, we can look back on the formulated functions for $L_{p}$ and $\sigma$ since it is possible that the data regarding $\mathrm{CRP}$ concentration, $L_{p}$, and $\sigma$ are insufficient to produce a good representation of the 
system. It may also be useful if more data about these values are available to improve the behavior of the formulated functions. Also, we can reconsider other means to quantify inflammation such as measuring other biomarkers or a combination of markers. We may also need to examine the inflammation process in more detail. We realize that incorporating inflammation is not so simple and devising a way to quantify inflammation is a real challenge. At the moment, we lack actual measurement data from experiments which are necessary in the parameter estimation method. Actual measurements from model outputs are also be needed in future studies. Although we are interested in local identifiability of parameters, we also suggest looking at global minimization methods in obtaining parameter estimates. We have seen that combining local and global search methods significantly improve the estimates. Finally, we have assumed that only the five parameters $a, L, d, b, c$ change as the CRP levels change and that the rest of the parameters remain fixed. Other parameters such as the rate of lymph flow and the protein concentration of the lymph may change as the degree of inflammation changes or as the length of treatment time increases. Hence, it may be useful to review this assumption and determine up to when it is valid. 



\section{Appendix A}

\section{Equilibrium Points}

Our proposed model is a modification of the model by de los Reyes et al. [13 wherein the parameters $L_{p}$ and $\sigma$ are made functions of the inflammatory marker concentration $[C R P]$, but not with time $t$. Hence, the equilibrium points of the proposed model is the same as those in [13]. It has been presented in the appendix of [13], that the model has two equilibrium points $\left(V_{p}^{*}, c_{p, 1}^{*}, V_{i}^{*}, c_{i, 1}^{*}\right)$ and $\left(V_{p}^{*}, c_{p, 2}^{*}, V_{i}^{*}, c_{i, 2}^{*}\right)$. Below we show the calculations.

Denote by $\left(V_{p}^{*}, c_{p}^{*}, V_{i}^{*}, c_{i}^{*}\right)$, an equilibrium point of the proposed model (2.5). Note that $V_{p}^{*}, c_{p}^{*}, V_{i}^{*}$, and $c_{i}^{*}$ should all be positive for the equilibrium point to be physiologically meaningful. To solve for $\left(V_{p}^{*}, c_{p}^{*}, V_{i}^{*}, c_{i}^{*}\right)$, we equate the model equations to zero.

$$
\begin{aligned}
J_{v}+\kappa-J_{U F} & =0 \\
\frac{J_{s}-c_{p}\left(J_{v}+\kappa-J_{U F}\right)}{V_{p}} & =0 \\
-J_{v}-\kappa & =0 \\
\frac{-J_{s}+c_{i}\left(J_{v}+\kappa\right)}{V_{i}} & =0
\end{aligned}
$$

Combining A.1 and A.3 we get that $J_{U F}=0$. It is easily seen from A.3 that $J_{v}=-\kappa$. Also, both $(\mathrm{A} .2)$ and (A.4) imply that $J_{s}=0$. Since $\kappa>0$, then $J_{v}<0$. From $J_{s}=0$ and $J_{v}<0$, it follows that

$$
\begin{aligned}
\underbrace{J_{v}}_{-\kappa}(1-\sigma)\left(c_{p}^{*}-\frac{c_{p}^{*}-c_{i}^{*}}{e^{x^{*}}-1}\right) & =-\alpha \kappa \\
\Leftrightarrow c_{p}^{*}\left(e^{x^{*}}-1\right)-\left(c_{p}^{*}-c_{i}^{*}\right) & =\frac{\alpha\left(e^{x^{*}}-1\right)}{1-\sigma} \\
\Leftrightarrow c_{i}^{*} & =\frac{\alpha\left(e^{x^{*}}-1\right)}{1-\sigma}-c_{p}^{*}\left(e^{x^{*}}-2\right),
\end{aligned}
$$

where $x^{*}=\frac{-\alpha(1-\sigma)}{P s}$ is a constant. Hence, $e^{x^{*}}$ is also a constant. We simplify the 
expression for $c_{i}^{*}$ obtained above by letting $Y:=\frac{\alpha\left(e^{x^{*}}-1\right)}{1-\sigma}$ and $Z:=e^{x^{*}}-2$. Rewriting, we have

$$
c_{i}^{*}=Y-c_{p}^{*} Z
$$

Next, we look for an expression that gives $c_{p}^{*}$ explicitly. Using the equation for $J_{v}$ given by (2.6), $J_{v}=-\kappa$, and (A.5),

$$
\begin{aligned}
\sigma\left(\pi_{p}-\pi_{i}\right)-\left(P_{c}-P_{i}\right) & =-\frac{\kappa}{L_{p}} \\
\sigma\left(a_{p, 1} c_{p}^{*}+a_{p, 2}\left(c_{p}^{*}\right)^{2}\right)-\sigma\left(a_{i, 1} c_{i}^{*}+a_{i, 2}\left(c_{i}^{*}\right)^{2}\right) & =-\frac{\kappa}{L_{p}}+\left(P_{c}-P_{i}\right) \\
\sigma\left(a_{p, 1} c_{p}^{*}+a_{p, 2}\left(c_{p}^{*}\right)^{2}\right)-\sigma a_{i, 1}\left(Y-c_{p}^{*} Z\right)-\sigma a_{i, 2}\left(Y-c_{p}^{*} Z\right)^{2} & =-\frac{\kappa}{L_{p}}+\left(P_{c}-P_{i}\right) \\
\left(c_{p}^{*}\right)^{2}\left(\sigma a_{p, 2}-\sigma a_{i, 2} Z^{2}\right)+c_{p}^{*}\left(\sigma a_{p, 1}+\sigma a_{i, 1} Z+2 \sigma a_{i, 2} Y Z\right)+ & \\
\left(\frac{\kappa}{L_{p}}-\left(P_{c}-P_{i}\right)-\sigma a_{i, 1} Y-\sigma a_{i, 2} Y^{2}\right) & =0 .
\end{aligned}
$$

Denote the coefficients of $\left(c_{p}^{*}\right)^{2}, c_{p}^{*}$, and the constant in the quadratic equation above by

$$
\begin{aligned}
U & :=\sigma a_{p, 2}-\sigma a_{i, 2} Z^{2}=\sigma a_{p, 2}-\sigma a_{i, 2}\left(e^{x^{*}}-2\right)^{2} \\
W & :=\sigma a_{p, 1}+\sigma a_{i, 1} Z+2 \sigma a_{i, 2} Y Z \\
& =\sigma a_{p, 1}+\sigma a_{i, 1}\left(e^{x^{*}}-2\right)+2 \sigma a_{i, 2}\left(\frac{\alpha\left(e^{x^{*}}-1\right)}{1-\sigma}\right)\left(e^{x^{*}}-2\right) \\
R & :=\frac{\kappa}{L_{p}}-\left(P_{c}-P_{i}\right)-\sigma a_{i, 1} Y-\sigma a_{i, 2} Y^{2} \\
& =\frac{\kappa}{L_{p}}-\left(P_{c}-P_{i}\right)-\sigma a_{i, 1}\left(\frac{\alpha\left(e^{x^{*}}-1\right)}{1-\sigma}\right)-\sigma a_{i, 2}\left(\frac{\alpha\left(e^{x^{*}}-1\right)}{1-\sigma}\right)^{2},
\end{aligned}
$$

respectively. Then there are two explicit expressions for $c_{p}^{*}$ and they are given by

$$
\begin{aligned}
& c_{p, 1}^{*}=\frac{-W+\sqrt{W^{2}-4 U R}}{2 U} \quad \text { and } \\
& c_{p, 2}^{*}=\frac{-W-\sqrt{W^{2}-4 U R}}{2 U} .
\end{aligned}
$$

The corresponding expressions for $c_{i, 1}^{*}$ and $c_{i, 2}^{*}$ are

$$
\begin{aligned}
& c_{i, 1}^{*}=\frac{\alpha\left(e^{x^{*}}-1\right)}{1-\sigma}-c_{p, 1}^{*}\left(e^{x^{*}}-2\right) \\
& c_{i, 2}^{*}=\frac{\alpha\left(e^{x^{*}}-1\right)}{1-\sigma}-c_{p, 2}^{*}\left(e^{x^{*}}-2\right) .
\end{aligned}
$$

Finally, we note that the only condition for both $V_{p}^{*}$ and $V_{i}^{*}$ is that they are positive. 


\section{Bibliography}

[1] AC Guyton and JE Hall. Textbook of Medical Physiology 11th Edition. Philadelphia, Pennsylvania: Elsevier Inc., 2006.

[2] Kidney Disease: Improving Global Outcomes (KDIGO) CKD Work Group. KDIGO 2012 Clinical Practice Guideline for the Evaluation and Management of Chronic Kidney Disease. 2013.

[3] Zoccali C, Kramer A, and Jager KJ. "Chronic kidney disease and end-stage renal disease - a review produced to contribute to the report "the status of health in the European union: towards a healthier Europe"'. In: NDT Plus 3 (2010), pp. 213224. DOI: 10.1093/ndtplus/sfp127.

[4] Stenvinkel P. "Inflammation in end-stage renal disease - a fire that burns within". In: Contrib Nephrol. 149 (2005), pp. 185-199.

[5] Kato S, Chmielewski M, Honda H, Pecoits-Filho R, Matsuo S, Yuzawa Y, Tranaeus A, Stenvinkel P, and Lindholm B. "Aspects of immune dysfunction in end-stage renal disease". In: Clinical Journal of the American Society of Nephrology 3.5 (2008), pp. 1526-33.

[6] Yao Q, Axelsson J, Stenvinkel P, and Lindholm B. "Chronic systemic inflammation in dialysis patients: an update on causes and consequences". In: ASAIO J 50 (2004), pp. lii-lvii.

[7] Menon V, Gul A, and Sarnak MJ. "Cardiovascular risk factors in chronic kidney disease". In: Kidney Int. 68 (2005), pp. 1413-1418.

[8] Stenvinkel P, Carrero JJ, Axelsson J, Lindholm B, Heimbürger O, and Massy Z. "Emerging biomarkers for evaluating cardiovascular risk in the chronic kidney disease patient: How do new pieces fit into the uremic puzzle?" In: Clin K Am Soc Nephrol 3 (2008), pp. 505-521.

[9] Herzog CA, Asinger RW, Berger AK, Charytan DM, Díez J, Hart RG, Eckardt KU, Kasiske BL, McCullough PA, Passman RS, DeLoach SS, Pun PH, and Ritz E. "Cardiovascular disease in chronic kidney disease. A clinical update from Kidney Disease: Improving Global Outcomes (KDIGO)". In: Kidney Int. 80.6 (2011), pp. $572-86$. 
[10] Stenvinkel P and Alvestrand A. "Inflammation in end-stage renal disease: sources, consequences, and therapy". In: Semin Dial 15 (2002), pp. 329-337.

[11] Stenvinkel P. "Inflammation in end-stage renal failure: could it be treated?" In: Nephrol Dial Transplant. 17.Suppl 8 (2002), pp. 33-38.

[12] Stenvinkel P. "Inflammation in end-stage renal disease: the hidden enemy". In: Nephrology (Carlton) 11.1 (2006), pp. 36-41.

[13] de los Reyes V AA, Fuertinger DH, Kappel F, Meyring-Wösten A, Thijssen S, and Kotanko P. "A physiologically based model of vascular refilling during ultrafiltration in hemodialysis". In: J Theor Biol. 390 (2016), pp. 146-155.

[14] Ridker PM. "C-Reactive Protein: Eighty Years from Discovery to Emergence as a Major Risk Marker for Cardiovascular Disease". In: Clin Chem. 55.2 (2009), pp. 209-215.

[15] SS Mader. Biology 10th Ed. Chapter 32: Circulation \& Cardiovascular Systems. Copyright The McGraw Hill Companies Inc. Retrieved June 23, 2016, from http: //slideplayer.com/slide/6655746/, 2009.

[16] Waterhouse J, Sawdon M, and Kirkman E. "Capillary dynamics and the interstitial fluid - lymphatic system". In: Anaesthesia and Intensive Care Medicine 14.2 (2013), pp. 72-78.

[17] Depiction of the major types of capillaries, showing fenestrations as well as intercellular gaps. Capillary, In Wikipedia. Retrieved June 23, 2016, from https: //en.wikipedia.org/wiki/Capillary.

[18] Taylor AE. "Capillary fluid filtration. Starling forces and lymph flow". In: Circulation Research 49.3 (1981), pp. 557-75.

[19] Levick JR and Michel CC. "Microvascular fluid exchange and the revised Starling principle". In: Cardiovasc Res. 87.2 (2010), pp. 198-210.

[20] Hemodialysis System. Retrieved June 23, 2016, from http : / / amcclibrary . blogspot.co.at/2012/07/hemodialysis-system.html.

[21] Koomans HA, Geers AB, and Mees EJ. "Plasma volume recovery after ultrafiltration in patients with chronic renal failure". In: Kidney Int. 26.6 (1984), pp. 84854 .

[22] Chamney PW, Johner C, Aldridge C, Krämer M, Valasco N, Tattersall JE, Aukaidey T, Gordon R, and Greenwood RN. "Fluid balance modelling in patients with kidney failure". In: J Med Eng Technol 23.2 (1999), pp. 45-52.

[23] Thijssen S, Kappel F, and Kotanko P. "Absolute blood volume in hemodialysis patients: why is it relevant, and how to measure it?" In: Blood Purif. 35.1-3 (2013), pp. $63-71$. 
[24] Carrero JJ and Stenvinkel P. "Persistent inflammation as a catalyst for other risk factors in chronic kidney disease: a hypothesis proposal". In: Clinical Journal of the American Society of Nephrology 4 (2009), S49-S55.

[25] Yilmaz MI, Carrero JJ, Axelsson J, Lindholm B, and Stenvinkel P. "Low-grade inflammation in chronic kidney disease patients before the start of renal replacement therapy: sources and consequences". In: Clinical Nephrology 68.1 (2007), pp. 1-9.

[26] Stenvinkel P. "The role of inflammation in the anaemia of end-stage renal disease". In: Nephrology Dialysis Transplant 16.[Suppl 7] (2001), pp. 36-40.

[27] Stenvinkel P. "Interactions between inflammation, oxidative stress, and endothelial dysfunction in end-stage renal disease". In: Journal of Renal Nutrition 13.2 (2003), pp. 144-148.

[28] Zimmermann J, Herrlinger S, Pruy A, Metzger T, and Wanner C. "Inflammation enhances cardiovascular risk and mortality in hemodialysis patients". In: Kidney International 55 (1999), pp. 648-658.

[29] Zoccali C, Tripepi G, and Mallamaci F. "Dissecting inflammation in ESRD: do cytokines and C-reactive protein have a complementary prognostic value for mortality in dialysis patients?" In: J Am Soc Nephrol. 17.12 Suppl 3 (2006), S16973.

[30] Miyamoto T, Carrero JJ, and Stenvinkel P. "Inflammation as a risk factor and target for therapy in chronic kindney disease". In: Current Opinion in Nephrology and Hypertension 20.6 (2011), pp. 662-668.

[31] Machowska A, Carrero JJ, Lindholm B, and Stenvinkel P. "Therapeutics targeting persistent inflammation in chronic kidney disease". In: Transl Res. 167.1 (2016), pp. 204-213.

[32] Meuwese CL, Carrero JJ, and Stenvinkel P. "Recent insights in inflammationassociated wasting in patients with chronic kidney disease". In: Contrib Nephrol. 171 (2011), pp. 120-126.

[33] Carrero JJ and Stenvinkel P. "Inflammation in End-Stage Renal Disease - What Have We Learned in 10 Years?" In: Seminars in Dialysis 23.5 (2010), pp. 498-509.

[34] Pecoits-Filho R, Lindholm B, and Stenvinkel P. "The malnutrition, inflammation, and atherosclerosis (MIA) syndrome - the heart of the matter". In: Nephrol Dial Transplant. 17.Suppl 11 (2002), pp. 28-31.

[35] Cobo G, Qureshi AR, Lindholm B, and Stenvinkel P. "C-reactive protein: repeated measurements will improve dialysis patient care". In: Semin Dial. (2015). DoI: doi:10.1111/sdi.12440. 
[36] Yao Q, Axelsson J, Stenvinkel P, and Lindholm B. "Systemic inflammation in dialysis patients with end-stage renal disease: causes and consequences". In: Minerva Urol Nefrol 56.3 (2004), pp. 237-48.

[37] Jofré R, Rodriguez-Benitez P, López-Gómez JM, and Pérez-Garcia R. "Inflammatory Syndrome in Patients on Hemodialysis". In: Journal of the American Society of Nephrology 17 (2006), S274-S280.

[38] Zsom L, Zsom M, Fulop T, and Flessner MF. "Treatment time, chronic inflammation, and hemodynamic stability: the overlooked parameters in hemodialysis quantification". In: Semin Dial. 21.5 (2008), pp. 395-400.

[39] Bazeley J, Bieber B, Li Y, Morgenstern H, de Sequera P, Combe C, Yamamoto H, Gallagher M, Port FK, and Robinson BM. "C-reactive protein and prediction of 1-year mortality in prevalent hemodialysis patients". In: Clin J Am Soc Nephrol 6.10 (2011), pp. 2452-61.

[40] Stenvinkel P, Heimb urger O, Paultre F, Diczfalusy U, Wang T, Berglund L, and Jogestrand T. "Strong association between malnutrition, inflammation, and atherosclerosis in chronic renal failure". In: Kidney Int. 55.5 (1999), pp. 18991911.

[41] Stenvinkel P, Gillespie IA, Tunks J, Addison J, Kronenberg F, Drueke TB, Marcelli D, Schernthaner G, Eckardt KU, Floege J, Froissart M, Anker SD, and ARO Steering Committee. "Inflammation Modifies the Paradoxical Association between Body Mass Index and Mortality in Hemodialysis Patients". In: J Am Soc Nephrol. 27 (2015).

[42] Kaysen GA. "C-Reactive Protein: a story half told". In: Semin in Dial. 13.3 (2000), pp. $143-146$.

[43] Zwaka TP, Hombach V, and Torzewski J. "C-reactive protein-mediated low density lipoprotein uptake by macrophages: implications for atherosclerosis". In: Circulation 103.9 (2001), pp. 1194-1197.

[44] Sandkamp M, Funke H, Schulte H, Köhler E, and Assmann. "Lipoprotein(a) is an independent risk factor for myocardial infarction at a young age". In: Clin Chem. 36.1 (1990), pp. 20-3.

[45] Smith EB and Thompson WD. "Fibrin as a factor in atherogenesis". In: Thromb Res. 73.1 (1994), pp. 1-19.

[46] Huber SA, Sakkinen P, Conze D, Hardin N, and Tracy R. "Interleukin-6 exacerbates early atherosclerosis in mice". In: Arterioscler Thromb Vasc Biol. 19.10 (1999), pp. 2364-7. 
[47] Ridker PM, Rifai N, Stampfer MJ, and Hennekens CH. "Plasma concentration of interleukin- 6 and the risk of future myocardial infarction among apparently healthy men". In: Circulation 101.15 (2000), pp. 1767-72.

[48] Harris TB, Ferrucci L, Tracy RP, Corti MC, Wacholder S, Ettinger WH Jr, Heimovitz H, Cohen HJ, and Wallace R. "Associations of elevated interleukin6 and C-reactive protein levels with mortality in the elderly". In: Am J Med. 106.5 (1999), pp. 506-12.

[49] Zuckerman SH and O'Neal L. "Endotoxin and GM-CSF-mediated down-regulation of macrophage apo E secretion is inhibited by a TNF-specific monoclonal antibody". In: J Leukoc Biol. 55.6 (1994), pp. 743-8.

[50] Tintut Y, Patel J, Parhami F, and Demer LL. "Tumor necrosis factor-alpha promotes in vitro calcification of vascular cells via the cAMP pathway". In: Circulation 102.21 (2000), pp. 2636-42.

[51] Levine B, Kalman J, Mayer L, Fillit HM, and Packer M. "Elevated circulating levels of tumor necrosis factor in severe chronic heart failure". In: $N$ Engl J Med. 323.4 (1990), pp. 236-41.

[52] Akchurin OM and Kaskel F. "Update on inflammation in chronic kidney disease". In: Blood Purif. 39.1-3 (2015), pp. 84-92.

[53] Meuwese CL, Stenvinkel P, Dekker FW, and Carrero JJ. "Monitoring of inflammation in patients on dialysis: forewarned is forearmed". In: Nat Rev Nephrol. 7 (2011), pp. 166-176.

[54] Stinghen AE, Bucharles, Riella MC, and Pecoits-Filho R. "Immune mechanisms involved in cardiovascular complications of chronic kidney disease". In: Blood Purif. 29.2 (2010), pp. 114-120.

[55] Tripepi G, Mallamaci F, and Zoccali C. "Inflammation Markers, Adhesion Molecules, and All-Cause and Cardiovascular Mortality in Patients with ESRD: Searching for the Best Risk Marker by Multivariate Modeling". In: Journal of the American Society of Nephrology 16 (2005), S83-S88.

[56] Lacson E Jr and Levin NW. "C-reactive protein and end-stage renal disease". In: Semin Dial. 17.6 (2004), pp. 438-448.

[57] van der Sande FM, Kooman JP, and Leunissen KM. "The predictive value of Creactive protein in end-stage renal disease: is it clinically significant?" In: Blood Purif. 24.4 (2006), pp. 335-341.

[58] Vigushin DM, Pepys MB, and Hawkins PN. "Metabolic and scintigraphic studies of radioiodinated human C-reactive protein in health and disease". In: J Clin Invest. 91.4 (1993), pp. 1351-7. 
[59] Arici M and Walls J. "End-stage renal disease, atherosclerosis, and cardiovascular mortality: is C-reactive protein the missing link?" In: Kidney Int. 59.2 (2001), pp. $407-414$.

[60] Wanner C, Zimmerman J, Schwedler S, and Metzger T. "Inflammation and cardiovascular risk in dialysis patients". In: Kidney International 61 (2002), S99S102.

[61] Menon V, Greene T, Wang X, Pereira AA, Marcovina SM, Beck GJ, Kusek JW, Collins AJ, Levey AS, and Sarnak MJ. "C-reactive protein and albumin as predictors of all-cause and cardiovascular mortality in chronic kidney disease". In: Kidney Int. 68.2 (2005), pp. 766-772.

[62] Panichi V, Rizza GM, Paoletti S, Bigazzi R, Aloisi M, Barsotti G, Rindi P, Donati G, Antonelli A, Panicucci E, Tripepi G, Tetta C, Palla R, and RISCAVID Study Group. "Chronic inflammation and mortality in haemodialysis: effect of different renal replacement therapies. Results from the RISCAVID study". In: Nephrol Dial Transplant. 23.7 (2008), pp. 2337-2343.

[63] Nascimento MM, Pecoits-Filho R, Qureshi AR, Hayashi SY, Manfro RC, Pachaly MA, Renner L, Stenvinkel P, Lindholm B, and Riella MC. "The prognostic impact of fluctuating levels of C-reactive protein in Brazilian haemodialysis patients: a prospective study". In: Nephrol Dial Transplant. 19.11 (2004), pp. 2803-2809.

[64] den Elzen WP, van Manen JG, Boeschoten EW, Krediet RT, and Dekker FW. "The effect of single and repeatedly high concentrations of C-reactive protein on cardiovascular and non-cardiovascular mortality in patients starting with dialysis". In: Nephrol Dial Transplant. 21.6 (2006), pp. 1588-1595.

[65] DN Granger and E Senchenkova. Inflammation and the Microcirculation. http: //www.ncbi.nlm.nih.gov/books/NBK53373/?report=classic San Rafael (CA): Morgan \& Claypool Life Sciences, 2010.

[66] J Scallan, VH Huxley, and RJ Korthuis. Capillary Fluid Exchange: Regulation, Functions, and Pathology. http://www.ncbi.nlm.nih.gov/books/NBK53447/ San Rafael (CA): Morgan \& Claypool Life Sciences, 2010.

[67] Cotran RS. "The Delayed and Prolonged Vascular Leakage in Inflammation II. An Electron Microscopy Study of the Vascular Response after Thermal Injury". In: Am J Pathol. 46 (1965), pp. 589-620.

[68] Clough G, Michel CC, and Phillips ME. "Inflammatory changes in permeability and ultrastructure of single vessels in the frog mesenteric microcirculation". In: $J$ Physiol. 395 (1988), pp. 99-114.

[69] Michel CC and Curry FE. "Microvascular permeability". In: Physiological Reviews 79.3 (1999), pp. 703-61. 
[70] Durán WN, Breslin JW, and Sánchez FA. "The NO cascade, eNOS location, and microvascular permeability". In: Cardiovasc Res. 87.2 (2010), pp. 254-61.

[71] Reed RK and Rubin K. "Transcapillary exchange: role and importance of the interstitial fluid pressure and the extracellular matrix". In: Cardiovasc Res. 87.2 (2010), pp. 211-217.

[72] Bates DO. "Vascular endothelial growth factors and vascular permeability". In: Cardiovasc Res. 87.2 (2010), pp. 262-271.

[73] Kaysen GA, Dubin JA, Müller HG, Rosales LM, and Levin NW. "The acutephase response varies with time and predicts serum albumin levels in hemodialysis patients. The HEMO Study Group". In: Kidney Int. 58.1 (2000), pp. 346-352.

[74] Meuwese CL, Snaedal S, Halbesma N, Stenvinkel P, Dekker FW, Qureshi AR, Barany P, Heimburger O, Lindholm B, Krediet RT, Boeschoten EW, and Carrero JJ. "Trimestral variations of C-reactive protein, interleukin-6 and tumour necrosis factor- $\alpha$ are similarly associated with survival in haemodialysis patients". In: Nephrol Dial Transplant. 26.4 (2011), pp. 1313-1318.

[75] Eknoyan G, Levey AS, Beck GJ, Agodoa LY, Daugirdas JT, Kusek JW, Levin NW, and Schulman G. "The Hemodialysis (HEMO) Study: Rationale for Selection of Interventions". In: Semin Dial. 9.1 (1996), pp. 24-33.

[76] Greene T, Beck GJ, Gassman JJ, Gotch FA, Kusek JW, Levey AS, Levin NW, Schulman G, and Eknoyan G. "Design and statistical issues of the hemodialysis (HEMO) study". In: Control Clin Trials 21.5 (2000), pp. 502-25.

[77] Carrero JJ, Qureshi AR, Axelsson J, Avesani CM, Suliman ME, Kato S, Bárány P, Snaedal-Jonsdottir S, Alvestrand A, Heimbürger O, Lindholm B, and Stenvinkel P. "Comparison of nutritional and inflammatory markers in dialysis patients with reduced appetite". In: Am J Clin Nutr. 85.3 (2007), pp. 695-701.

[78] Termorshuizen F, Korevaar JC, Dekker FW, Van Manen JG, Boeschoten EW, Krediet RT, and Netherlands Cooperative Study on the Adequacy of Dialysis Study Group. "Hemodialysis and peritoneal dialysis: comparison of adjusted mortality rates according to the duration of dialysis: analysis of The Netherlands Cooperative Study on the Adequacy of Dialysis 2". In: J Am Soc Nephrol. 14.11 (2003), pp. 2851-60.

[79] Korevaar JC, van Manen JG, Dekker FW, de Waart DR, Boeschoten EW, Krediet RT, and NECOSAD study group. "Effect of an increase in C-reactive protein level during a hemodialysis session on mortality". In: J Am Soc Nephrol. 15.11 (2004), pp. 2916-2922. 
[80] Meuwese CL, Halbesma N, Stenvinkel P, Dekker FW, Molanaei H, Qureshi AR, Barany P, Heimburger O, Lindholm B, Krediet RT, Boeschoten EW, and Carrero JJ. "Variations in C-reactive protein during a single haemodialysis session do not associate with mortality". In: Nephrol Dial Transplant. 25.11 (2010), pp. 3717-23.

[81] Schneditz D, Roob J, Oswald M, Moser M Pogglitsch H, Kenner T, and Binswanger U. "Nature and rate of vascular refilling during hemodialysis and ultrafiltration". In: Kidney Int. 42.6 (1992), pp. 1425-33.

[82] Yashiro M, Hamada Y, Matsushima H, and Muso E. "Estimation of Filtration Coefficients and Circulating Plasma Volume by Continuously Monitoring Hematocrit during Hemodialysis". In: Blood Purif. 20.6 (2002), pp. 569-576.

[83] Yashiro M, Watanabe H, Tomita M, Yamadori N, and Muso E. "Relationship between filtration coefficients of microvasculature and levels of atrial natriuretic peptide or echocardiographic measurements". In: Blood Purif. 23.6 (2005), pp. 431439 .

[84] Cotran RS and Majno G. "The Delayed and Prolonged Vascular Leakage in Inflammation I. Topography of the Leaking Vessels after Thermal Injury". In: Am J Pathol. 45.2 (1964), pp. 261-281.

[85] Levick JR. "Capillary filtration-absorption balance reconsidered in light of dynamic extravascular factors". In: Exp Physiol. 76.6 (1999), pp. 825-857.

[86] Cintrón-Arias A, Banks HT, Capaldi A, and Lloyd AL. "A sensitivity matrix based methodology for inverse problem formulation". In: J Inv Ill-Posed Problems 17.6 (2009), pp. 545-564.

[87] Banks HT, Dediu S, Ernstberger SL, and Kappel F. "Generalized sensitivities and optimal experimental design”. In: J Inv Ill-Posed Problems 18.1 (2010), pp. 25-83.

[88] Banks HT, Cintrón-Arias A, and Kappel F. "Parameter selection methods in inverse problem formulation". In: Mathematical Modeling and Validation in Physiology: Applications to the Cardiovascular and Respiratory Systems. Ed. by JJ Batzel, M Bachar, and F Kappel. Lecture Notes in Mathematics. Berlin Heidelberg: Springer-Verlag, 2013. Chap. 3, pp. 43-73.

[89] Kappel F and Munir M. "Generalized sensitivity functions for multiple output systems". 